\title{
Non-invasive arterial wall tissue characterization : development and evaluation of narrowband ultrasound techniques
}

Citation for published version (APA):

Linssen, F. M. J. (1992). Non-invasive arterial wall tissue characterization : development and evaluation of narrowband ultrasound techniques. [Doctoral Thesis, Maastricht University]. Rijksuniversiteit Limburg. https://doi.org/10.26481/dis.19920326fl

Document status and date:

Published: 01/01/1992

DOI:

10.26481/dis.19920326fl

Document Version:

Publisher's PDF, also known as Version of record

Please check the document version of this publication:

- A submitted manuscript is the version of the article upon submission and before peer-review. There can be important differences between the submitted version and the official published version of record.

People interested in the research are advised to contact the author for the final version of the publication, or visit the DOI to the publisher's website.

- The final author version and the galley proof are versions of the publication after peer review.

- The final published version features the final layout of the paper including the volume, issue and page numbers.

Link to publication

\footnotetext{
General rights rights.

- You may freely distribute the URL identifying the publication in the public portal. please follow below link for the End User Agreement:

www.umlib.nl/taverne-license

Take down policy

If you believe that this document breaches copyright please contact us at:

repository@maastrichtuniversity.nl

providing details and we will investigate your claim.
}

Copyright and moral rights for the publications made accessible in the public portal are retained by the authors and/or other copyright owners and it is a condition of accessing publications that users recognise and abide by the legal requirements associated with these

- Users may download and print one copy of any publication from the public portal for the purpose of private study or research.

- You may not further distribute the material or use it for any profit-making activity or commercial gain

If the publication is distributed under the terms of Article 25fa of the Dutch Copyright Act, indicated by the "Taverne" license above, 


\section{NON-INVASIVE \\ ARTERIAL WALL TISSUE \\ CHARACTERIZATION}

DEVELOPMENT AND EVALUATION OF NARROWBAND

ULTRASOUND TECHNIQUES 


\section{NON-INVASIVE \\ ARTERIAL WALL TISSUE \\ CHARACTERIZATION}

\section{DEVELOPMENT AND EVALUATION OF NARROWBAND ULTRASOUND TECHNIQUES}

\section{PROEFSCHRIFT}

ter verkrijging van de graad van doctor aan de Rijksuniversiteit Limburg te Maastricht, op gezag van de Rector Magnificus, Prof. mr. M.J. Cohen, volgens het besluit van het College van Decanen, in het openbaar te verdedigen op donderdag, 26 maart 1992 om 16.00 uur

door

Franciscus Maria Joannes Linssen

geboren te Echt op 31 December 1962 
Promotoren:

Prof. Dr. ir. A. Hasman

Prof. Dr. R.S. Reneman

Co-promotor: Dr. ir. A.P.G. Hoeks

Beoordelingscommissie: Prof. Dr. ir. drs. O.J. Vrieze (voorzitter)

Prof. Dr. ir. M.G.J. Arts (Universiteit Twente)

Prof. Dr. ir. N. Bom (Erasmus Universiteit Rotterdam)

Prof. Dr. H.J. van den Herik

Dr. ir. J.M. Thijssen (Katholieke Universiteit Nijmegen)

CIP-DATA KONINKLIIKE BIBLIOTHEEK, DEN HAAG

Linssen, Franciscus Maria Joamnes

Non-invasive arterial wall tissue characterization:

Development and evaluation of narrowband ultrasound techniques

Franciscus Maria Joannes Linssen. Maastricht [ill. by the author ].

Masstricht: Datawyse. - Ill.

Proefschrift Maastricht. - Met lit. opg. - Met samenvatting in het Nederlands.

ISBN 90-5291-074-X

Trefw: weefselkarakterizering / vaatziekten

Het verschijnen van dit proefschrift werd mede mogelijk gemaakt door financiële steun van:

- Pie Medical Benelux B.V.

- Nederlandse Hartstichting 
Aan mijn ouders 


\section{List of symbols}

\begin{tabular}{|c|c|}
\hline$a_{i}$ & coefficients \\
\hline A & amplitude \\
\hline c & velocity of sound \\
\hline $\mathrm{C}$ & constant (reflectivity, average attenuation etc.) \\
\hline$\gamma(0)$ & attenuation coefficient (function of frequency) \\
\hline $8(t)$ & Dirac function or delta function ( 8 -function) \\
\hline$d(t)$ & error signal or approximation of Dirac function \\
\hline Diff $(\zeta, t)$ & diffraction function \\
\hline$e(t)$ & electrical input signal \\
\hline$f_{r}(z)$ & sensitivity at point on transducer surface in reception \\
\hline$f_{t}(z)$ & sensitivity at point on transducer surface in transmission \\
\hline f & Erequency \\
\hline$\Phi(z, t)$ & velocity potential function at point with coordinate vector $\mathbf{z}$ \\
\hline$f(t)$ & system impulse response function \\
\hline $\ln (\mathbf{z}, \mathrm{t})$ & $\begin{array}{l}\text { diffraction impulse response function at point with coordinate } \\
\text { wector } z\end{array}$ \\
\hline$\lambda$ & wavelength \\
\hline$\omega$ & angular velocity \\
\hline $\mathrm{p}(\mathrm{z}, \mathrm{t})$ & pressure waveform at point with coordinate vector $z$ \\
\hline $\operatorname{Pr}(t)$ & electro-acoustical transfer function in reception \\
\hline$p_{t}(t)$ & electro-acoustical transfer function in transmission \\
\hline $\operatorname{PCF}(t)$ & Pressure Coupling Function \\
\hline $\mathrm{r}$ & $\begin{array}{l}\text { radial distance from central axis of sound beam or } \\
\text { distance between two points }\end{array}$ \\
\hline $\operatorname{RCF}(\mathrm{t})$ & Radiation Coupling Function \\
\hline RMS & Root-Mean-Square \\
\hline$\rho$ & density \\
\hline$s(t)$ & signal received from insonified tissue upon emission of a pulse \\
\hline SNR & Signal-to-Noise Ratio \\
\hline$v$ & noise cutooff level (constant) \\
\hline$v(1)$ & velocity waveform \\
\hline$v_{n}\left(z_{0}, t\right)$ & normal velocity at a point with coordinates $z_{0}$ at transducer surface \\
\hline$W(t)$ & waveform as received from a perfect point reflector \\
\hline$x_{n} y, z$ & coordinates, $z$ is generally chosen along the axis of the beam \\
\hline $\mathrm{z}$ & vector with coordinates of point \\
\hline Z & acoustic impedance \\
\hline 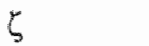 & average distance between region of interest and transducer \\
\hline
\end{tabular}

Symbols which are used locally only, are defined where necessary. 
Contents:

\section{Chapter 1 Introduction}

$\begin{array}{lll}1.1 & \text { The systemic circulation } & 11\end{array}$

1.2 Ultrasound: some basic definitions 13

$\begin{array}{lll}1.3 & \text { Detection of atherosclerotic lesions } & 18\end{array}$

1.4 Aim and outline of this thesis 22

\section{Chapter 2 Ultrasound signals}

2.1

2.2

2.3

2.4

2.5

2.6

Chapter 3 Estimation of transducer parameters from sound field measurements

3.1

3.2

3.3

3.3
Introduction

Mathematical model for ultrasound signals

27

Differentiation of tissues

Influence of Diffraction and Radiation Coupling

40

Conventional (broadband) and narrowband

ultrasound

41

Vessel wall tissue characterization
Introduction $\quad 49$

Sound field model and measurements 51

Results $\quad 57$

Discussion $\quad 57$

Appendix $\quad 61$

Chapter 4 Computation of Pressure Coupling Function

4.1

4.2

4.3

4.4

4.5
Introduction

Derivation of numerical model

Algorithm

Results

Discussion

Appendix
65

66

71

75

80

82

Chapter 5 NarrowBand Multi-Frequency method

5.1 
5.2 Theoretical background: convolution model and deconvolution $\quad 89$

$5.3 \quad$ Wiener-inverse filtering 91

5.4 Testing of the NarrowBand Multi-Frequency method 95

5.5 Discussion 99

$\begin{array}{ll}\text { Appendix } & 102\end{array}$

\section{Chapter 6 Influence of system transfer function}

$\begin{array}{lll}6.1 & \text { Introduction } & 107\end{array}$

6.2 Cause of frequency dependent bias in amplitude ratios 108

6.3 Improvement by using better deconvolution signals 110

$6.4 \quad$ Improvement of real ultrasound signals 114

6.5 Discussion 114

$\begin{array}{ll}\text { Appendix } & 117\end{array}$

Chapter 7 Evaluation of narrowband imaging and parameter estimation: preliminary in-vivo results

7.2

Equipment, data-handling and measurements

7.3

Imaging, attenuation and wall thickness

Chapter 8 Discussion, Conclusions and Recommendations

Summary

Samenvatting

Nawoord 
CHAPTER 1

INTRODUCTION 


\subsection{The systemic circulation}

\subsubsection{General description}

The blood circulatory system transports the oxygen and nutrients animal and human cells need to function. The pump of this system is the heart that ejects the blood into the aorta. The aorta is a large artery that branches into numerous smaller arteries. The arteries branch further down into very narrow vessels: the arterioles. These vessels branch into the capillaries which are the site of exchange of mutrients and waste products between blood and tissues. The blood leaving the capillaries is collected in venules, which drain into the network formed by the veins. These vessels transport the blood back to the heart (figure 1.1).

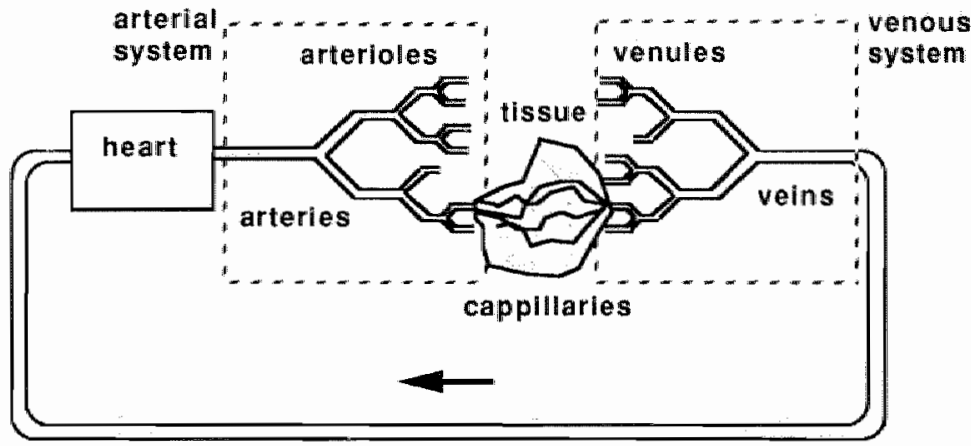

figure 1.1

Simplified model of the human systemic circulation (lung circulation omitted). The heart, the arterial tree, the tissues and the venous tree are drawn schematically. The flow direction of the blood is indicated.

The heart is a pulsatile pump. The arteries nearest to the heart are highly elastic and can be regarded as a windkessel. The windkessel effect of the arteries smoothes the peaks in the pressure, so that at the end of the arterial system the perfusion pressure is stabilized. Of course, this is a gross simplification, because there are many regulatory systems working both at the level of the large arteries and at the level of the microcirculation. The windkessel effect is not necessary in the venous system and a typical difference between a vein and an artery is that an artery wall contains a muscular (elastic) layer, whereas the wall of a vein mainly consists of connective tissues. The volume of blood is much larger in the venous system than in the arterial part of the circulation. The veins also have smooth muscle cells (although not confined within a distinct layer) which allow them to constrict and to redistribute blood from the venous to the arterial part of the circulation. By constriction of the veins the blood volume in the veins can be reduced by $15 \%$ and in that way the venous part of the circulatory system acts as a blood-volume regulator. The structure of an artery will be further described in the next section. 


\subsubsection{Artertes and atherosclerosis}

A schematic presentation of the structure of an artery is shown in figure 1.2 (Gross 1985). There are three main layers. The inner layer is the intima. The middle layer is the media and the outer layer is the adventitia. The intima consists of a mono-layer of endothelial cells, which are in direct contact with the blood in the lumen of the artery, a membrane (basement membrane) and the lamina propria, in which more or less randomly oriented smooth muscle cells and collagen fibers are found. Between the intima and the media there is the internal elastic membrane (tunica elastica interna). The media itself is a layer consisting of tangentially orientated smooth muscle cells with connective tissue in between. Between the media and the adventitia is the external elastic membrane (tunica elastica externa). On the outside the boundary of the adventitia is illdefined. There is a more or less continuous transition to the surrounding tissues. The adventitia consists of different tissues, but no muscle cells are found in this layer.

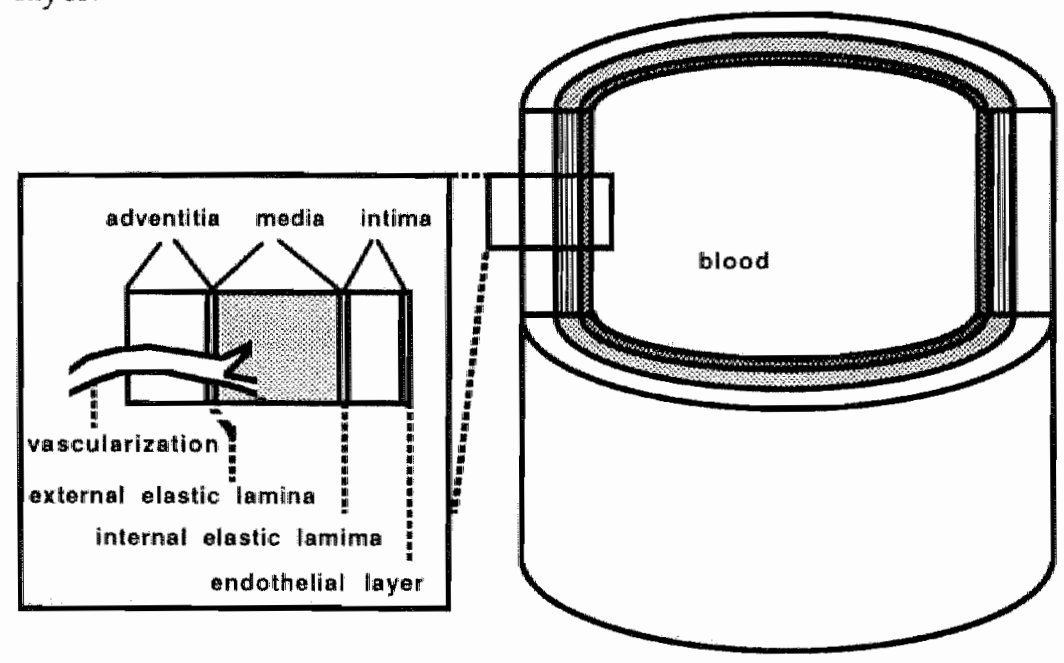

ligure 1.2 Schematic representation of an antery, showing three main layers, separated by elastic membranes.

Nutrients and oxygen are supplied to the artery walls through small vessels (vasa vasorum) which enter the vessel wall via the adventitia and end somewhere in the media. The intima and the inner part of the media receive their nutrients and oxygen directly from the blood in the lumen of the vessel. Problems in the arterial circulation are often caused by atherosclerotic processes. At early stages atherosclerosis only affects the composition of the artery wall. Till now, there is no complete theory about atherogenesis. Several deviations from the "normal" arterial wall can be observed even in young aduits at autopsy, but it is not clear which of these deviations can be considered to be harmless, and which are precursors of atherosclerotic lesions. Intimal thickening is one of the deviations from the "normal" situation and is commonly found in older sub- 
jects. Little spots containing lipids (fatty streaks), invasion of (modified) smooth muscle cells into the intima and accumulation of lipids and fibrous tissue in the wall (fibrous plaque formation) are also often found.

The (pre-atherosclerotic) deviations from the normal arterial wall are characterized by changes in composition and morphology of the arterial wall. Mostly, these early changes in the arterial wall do not lead to intraluminal processes and, therefore, do not influence the blood flow pattern. Only at later stages of the disease an atherosclerotic lesion may become so large that the lumen of the vessel is partly occluded. To obtain better insight into the natural history of atherogenesis and to improve early diagnosis of atherosclerosis, it is important to detect noninvasively early changes in composition and morphology of the arterial wall. Ultrasound is a non-invasive technique which can be used to this end. This technique is widely used to image superficial arteries, because, beside its noninvasiveness, ultrasound gives real-time information. Some regions of the body, however, cannot be imaged non-invasively by means of ultrasound. Intravascular transducers (mounted on catheters), for example, are used to investigate arteries from inside the lumen (Bom and Roelandt 1989). Ultrasound equipment is relatively cheap and therefore readily available. The principles of ultrasound and its possibilities with regard to the detection of atherosclerotic anomalities in the arterial system will be discussed in the following sections.

\subsection{Ultrasound: some basic definitions}

\subsubsection{Diffraction impulse response and ultrasound beam}

Ultrasound pulses are mechanical shock waves which are generated by applying an electrical excitation pulse to a piezo electric transducer. These shock waves travel into the region of the body in front of the transducer. In a certain point in front of the transducer (the sound field), pulses coming from different parts of a coherently vibrating transducer surface arrive at different times due to their difference in pathlength traveled (figure 1.3). The result is that these pulses interfere with each other. The velocity waveform, observed in a point of the sound field (coordinate vector $z$ ), upon emission of an infinitely short pulse ( $\delta$-function) at the transducer surface can be derived from a function, $h(z, t)$, which is called the diffraction impulse response function (Stepanishen 1971). The diffraction impulse response is a function of time which is different for every point of the sound field (in symmetrical soundfields there are points with the same $h(z, t))$. The pressure $p(z, t)$ is calculated from $h(z, t)$ and the emitted velocity waveform $v(t)$. The velocity waveform depends on the properties of the transducer and the electrical excitation signal e(t). A typical result for the pressure amplitude distribution in two planes, parallel to the transducer surface is depicted in figure 1.4. The pressure amplitude distribution can be calculated (or measured) in every perpendicular plane. The ultrasonic beam is then defined as the three dimensional region where the amplitude in a plane perpendicular to the axis of the beam is larger than a fraction (half) of the pressure peak amplitude in each parallel plane (see figure 1.4). The axis of the beam is the line through the positions where the pressure amplitude is maximum. 

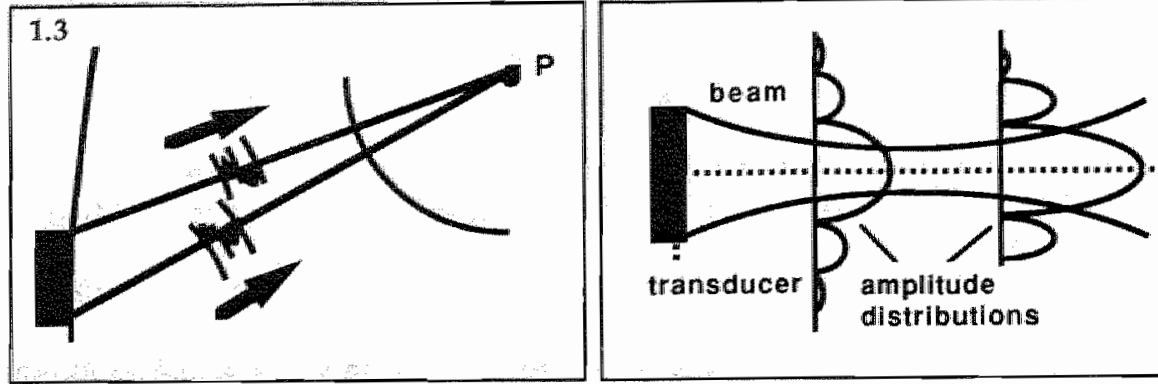

figure 1.3

The cause of diffraction is essentially the difference in pathlength for pulses originating from difterent points on the transducer surface to a certain point $P$ in the sound field. In the situation drawn in the figure, the pulse coming from the lower end of the transducer must travel a longer distance than a pulse coming from the upper part of the transducer. If the emitted pulse is a Dirac function (infinitely short pulse), the diffraction impulse response function, $h\left(z_{p}, t\right)$, in point $P$ (coordinates $z_{p}$ ) can be calculated.

figure 1.4 Schematic representation of a transducer and the ultrasound beam. The pressure amplitude distribution in two planes is shown. Here, the border of the ultrasound beam is drawn at the lateral position where the amplitude in the plane is half the maximum value. The axis of the ultrasound beam is the line through the positions in the planes where the pressure amplitude is maximum.

\subsubsection{Ultrasound imaging modes}

The principle of ultrasonic imaging is that a mechanical shock pulse, generated by a piezo electric transducer is reflected or scattered by structures in the body (Wells 1969). Reflection or scattering of sound waves occurs when a sound wave encounters a change in acoustic impedance (see section 1.2.3). Reflections from nearby structures are returned to the transducer surface earlier than those from structures which are further away. Analysis of the amplitude of the reflected signal as a function of the time of flight gives therefore information about the position and reflectivity of structures within the beam in front of the transducer (Amplitude or A-mode imaging, figure 1.5a). This procedure is repeated after moving the ultrasound beam (mechanically or electronically). The amplitude information obtained in each direction of the ultrasound beam is converted to inlensity levels on a TV screen. Because the orientation of the lines on the TV screen, where the amplitude information is displayed, corresponds with the actual orientation of the ultrasound beam, a two dimensional image of the (acoustical) structures inside the body is obtained (Brightness or B-mode imaging, figure $1.5 \mathrm{~b}$ ). Information about the motion of the structures along the axis of the ultrasonic beam is obtained by displaying the amplitude of the received signal on a TV screen with the beam oriented in the same direction. Subsequent echo lines are then displayed next to each other (Motion or M-mode imaging, figure $1.5 \mathrm{c}$ ). 

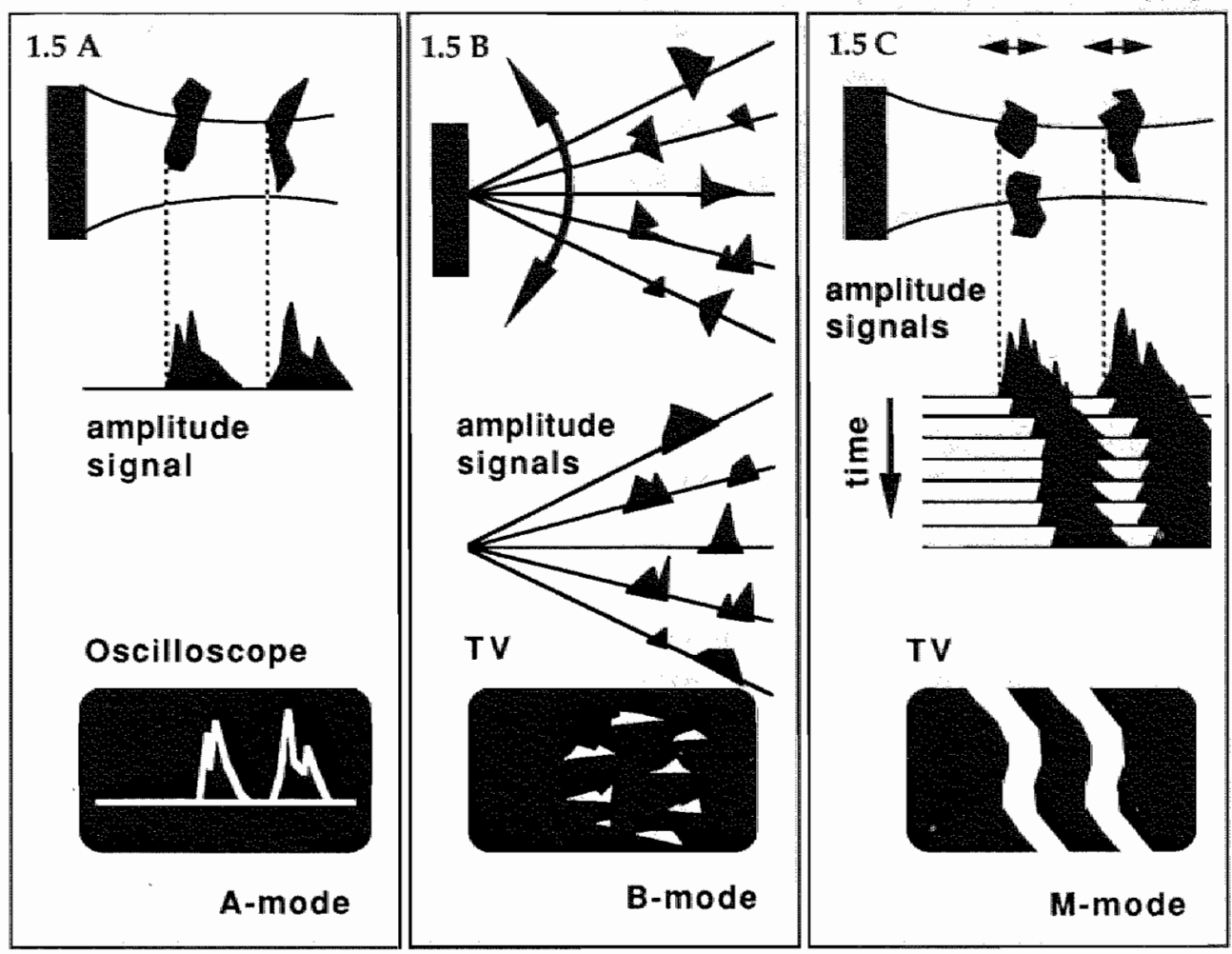

figure $1.5 \mathrm{a}$ : A-mode imaging (Amplitude mode). The amplitude of the received ultrasound signal is directly displayed on an oscilloscope. The transducer is kept in the same position, or moved gradually by hand, while observing the display of the oscilloscope.

figure 1.5b: B-mode imaging (Brightness mode). For every transducer direction, a pulse is emilled and the amplitude of the received ultrasound signal is transformed to a certain brightness along a line on a TV screen corresponding to the direction of the transducer. Here, the transducer is rotated it is also possible to move the transducer along a line or an arc.

figure 1.5c: M-mode imaging (Motion mode). The amplitude information is displayed as a brightness on a TV screen, but the transducer is kept in place. The amplitude of ultrasound signals received after emission of pulses at some Pulse Repetition Frequency (PRF) are displayed below each other. The image created in this way reveals the motion of the observed tissue structures. 


\subsubsection{Reflection and scattering * radiation coupling and diffraction effect}

The acoustic impedance $Z$ of a medium is determined by the phase velocity $c$ $\left[\mathrm{ms}^{-1}\right]$ of sound waves in that medium and the density $\rho\left[\mathrm{kg} \mathrm{m}^{-3}\right]$ of the medium:

$$
Z=\rho c
$$

The acoustic impedance depends on the type of tissue. If an acoustic wave travels across the interface of two tissues with different acoustic impedance, it follows from elementary mechanics that part of the acoustic wave is reflected and part is transmitted into the next tissue. This phenomenon, which typically occurs if the dimension of the interface parallel to the wavefront is much larger than the wavelength of the ultrasound waves, is called specular reflection (figure 1.6a).

Assuming a frequency independent sound velocity $c$, the wavelength $\lambda[\mathrm{m}]$ of the ultrasound signal depends on the carrier frequency $\mathrm{f}_{\mathrm{C}}\left[\mathrm{s}^{-1}\right]$ :

$$
\lambda=c / f_{c}
$$

For example, for an ultrasound signal with frequency $\mathrm{f}=5 \mathrm{MHz}$ and assuming a sound velocity of $1500 \mathrm{~ms}^{-1}$ the wavelength $\lambda$ equals $0.3 \mathrm{~mm}$. Because the ultrasound pulses arriving at the different points of the reflector depend on the shape and position of the reflector in the sound field, the characteristics of the ultrasound signal received upon reflection at a reflector (tissue interface) also depend on the shape and position of the reflector in the sound field. This phenomenon is called the radiation coupling effect.

If the dimension of the inhomogeneity (reflecting particle) is substantially smaller than the wavelength, the sound waves are scattered in all directions. Hence, this phenomenon is called scattering. In general the energy is not uniformly scattered in all directions. Especially if the dimension of the inhomogeneity is in the same order of magnitude as the wavelength, the directivity pattern of the scattering is very complicated (figure 1.6b). The characteristics of the ultrasound signal received from a certain tissue region depend on the distance between the transducer and the tissue region: the diffraction effect.

\subsubsection{Attenuation and backscattering}

The pressure amplitude of a pulse emilted by a point source decreases while traveling away from the source. Firstly, this is caused by the distribution of the acoustic energy over an increasing tissue volume. Secondly, some of the acoustic energy is absorbed in the medium through which the pulse travels (tissue). Thirdly, some energy is reflected in another direction than the direction of the ultrasound beam (reflection and scattering). It is difficult to discern the absorption and the scattering and the combined effect is called attenuation. 

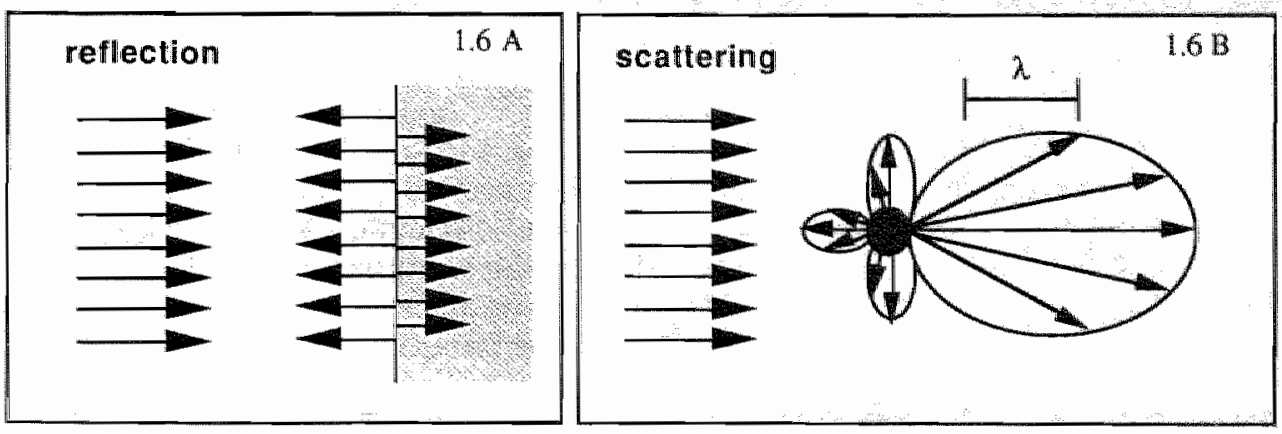

figure $1.6 \mathrm{a}$ : Reflection of a plane acoustic wavefront at a large tissue interface. The regions with different acoustic impedance are indicated.

figure 1.6b: Scaltering of a plane acoustic wavefront by a scatterer (small particle with larger or smaller acoustic impedance than the surrounding tissue. The acoustic waves, scattered in different directions, have different amplitude (directivity pattern).

For most biological tissues the attenuation coefficient $\gamma$ is approximately a linear function of frequency $f$ (Kuc 1979, Jones 1984).

$$
\gamma(f)=a_{0}+a_{1} f
$$

where $a_{0}\left[\mathrm{~m}^{-1}\right]$ and $\mathrm{a}_{1}\left[\mathrm{~m}^{-1} \mathrm{~s}\right]$ are constants. Then the amplitude of a plane wave with frequency $f$ is a function of the traveling distance $d$ according to:

$$
A(z=d)=A_{0} e^{-\gamma(f) d}
$$

where $A_{0}$ is the amplitude at $z=0$ (transducer surface). Especially the value of at (equation 1.3) is often used as a discriminating parameter in tissue characterization because it is relatively independent of the ultrasound equipment used.

Another tissue parameter used in the characterization of tissues is the backscattering coefficient. For the calculation of the backscattering coefficient a reference spectrum is needed. Ideally, the spectrum of the emitted pulse should be used as the reference spectrum. Mostly, however, the actually emitted pulse is not known and the reflection received from a plane reflector, placed in the focus of the ultrasound beam, is used as the reference spectrum. The ultrasound signal observed in a short time window corresponds to a limited tissue region (see chapter 2, section 2.1). The backscatter coefficient for a given frequency band is then defined as the ratio between the average amplitude spectrum of windowed signal and the average amplitude spectrum of the reference signal within that frequency band. 


\subsection{Detection of atherosclerotic lesions}

\subsubsection{Ulltrasound B-mode inaging}

The geometry of the vessel can be investigated with normal B-mode imaging, but until now the results do not seem to give accurate information about the composition of the wall. Especially the presence of "hypo" and "hyper echoic" zones in a B-mode image heavily depends on the equipment used (Green et al 1977). The limited axial resolution of echo systems and the complexity of the reflecting structures inside the vessel wall may be the reason that it is difficult to interpret $\mathrm{B}$-mode images. Calcified lesions are the only ones which can be dearly distinguished on a B-mode image, because they cause pronounced shadowing of the tissues behind the lesion. Using strict measurement protocols, however, it seems to be possible to measure the thickness of the layers in the common carotid artery using normal B-mode ultrasound images (Pignoli et al 1986, Poli et al 1988).

\subsubsection{Blood flow}

Investigation of the blood flow pattern for the detection of atherosclerotic lesions is a well known application of ultrasound. In Continuous Wave Doppler, sound waves are continuously emitted into the body from one transducer. The velocity of the blood particles in the ultrasound beam causes a Doppler shift of the backscattered signals which are registered by a second transducer, next to the emitting transducer. Continuous Wave Doppler gives information about the distribution of (blood) velocities in the ultrasound beam as a function of time. Because of the continuous emission of sound waves, this method does not provide information about the velocity profile in a blood vessel. Flow disturbances, caused by atherosclerotic lesions (narrowing of the lumen), can be detected by estimating the degree of spectral broadening of the Doppler spectrum (Reneman and Spencer 1979, Johnston et al 1981, Barnes et al 1982, Van Baalen et al 1984). Single-Gate Pulsed Doppler (Duplex scanning) was introduced to enable the investigation of blood flow in a limited region (sample volume) inside the blood vessel (Blackshear et al 1979, Breslau 1982). Multi-Gate Pulsed Doppler systems were introduced to study the distribution of the blood velocity along a single line through the vessel in detail (Peronneau 1974, Brandestini 1978, Hoeks et al 1981, Hoeks 1982). In this way the disturbances of the blood velocity profile due to (atherosclerotic) anomalities can be investigated directly (van Merode et al 1988 and 1989). This technique was further developed and resulted in Color Flow Doppler devices which give velocity information (coded in color) in a two dimensional plane across or along a vessel (or in the heart). All these Doppler techniques, however, cannot be used to diagnose early stages of the atherosclerotic process because deviations in blood flow pattern occur only at later stages of the disease, when the geometry of the vessel has been significantly affected (van Merode 1986). In regions with complicated flow patterns, phenomena like spectral broadening are observed in a normal situation and therefore, such phenomena cannot be used to detect minor lesions (Reneman et al, 1985). Only by combining the detection of blood flow disturbances and changes in vessel wall 
motion (see section 1.3.3.), accurate diagnosis of minor lesions (1-29\% obstruction of the lumen) can be achieved (van Merode et al 1989).

\subsubsection{Vessel wall motion}

Changes in composition and morphology may alter the mechanical properties of the vessel wall. Because the motion of the vessel walls due to the pulsatile na ture of pressure depends on these mechanical properties, techniques were introduced which allow detection of vessel wall motion. In combination with the pulse pressure it is then possible to determine changes in the mechanical stiffness of the artery (Reneman et al 1986). The earliest methods used the envelope of the received radio frequency signal (Hokanson et al 1972). Hoeks et al (1990) developed a Doppler approach based on tracking the position of the vessel wall using the radio frequency signal (RF-signal). Although deviations in mechanical behavior can be found at early stages of atherosclerosis (Van Merode ef al 1989), these deviations still do not yield direct information about the composition of an artery wall. For example, the distensibility coefficient (DC), which can be assessed with the beforementioned method, is a measure for the overall stiffness of the arterial wall (Reneman et al 1986):

$$
D C=\frac{\Delta d / d}{\Delta p}
$$

where $d$ is the diameter of the vessel, and $\Delta d$ is the change in diameter due to the pulsation of the pressure $\Delta p$.

The overall stiffness of a structure like the arterial wall, however, does not only depend on the properties of the material, but also on the thickness of the constituting layers. In a simplified approach, the distensibility coefficient is inversely proportional to the thickness $w$ [mm] and the modulus of elasticity $\mathrm{E}\left[\mathrm{N} \mathrm{m}^{-2}\right]$ of the material of the wall (see McDonald 1974, chapter 10):

$$
\mathrm{DC} \sim \mathbb{1} /(\mathrm{W} E)
$$

A lower distensibility coefficient (DC) may be the result of a tougher material ( $E$ is larger, e.g. more collagen fibers), or a thicker layer of the same material ( $w$ larger). From measurement of the mechanical properties of the vessel (DC) it is therefore not possible to discriminate between geometrical changes (thickness) and changes in composition (lissues with different elasticity).

\subsubsection{Tissue characterization}

The composition of the vessel wall, the existence of different types of tissue, and the acoustic properties (e.g. attenuation and backscaltering) of the composing tissues are affected by atherosclerosis (Landini et al 1985 and 1986, Sarnelli et al 1986). Detailed information about the composition of the vessel wall can be derived from ultrasonic tissue characterization techniques (Jones and Leeman 1984). The frequency dependence of the acoustic attenuation and backscattering 
strength may be discriminating parameters for several tissues and pathologies. Kuc et al (1979) reported good correlation between acoustic attenuation parameters and some liver pathologies. For thin layered structures, like an arterial wall, only in vitro data on attenuation and backscattering are presently available (Landini et al 1985 and 1986, Sarnelli et al 1986). Marked differences in several acoustic parameters were found for different pathologies. In wiwo determination of acoustic parameters of thin tissue layers, however, is a difficult problem. Bmode images of arteries do not always show the layered appearance that might be expected. Only if the orientation of the ultrasound beam is perpendicular to the vessel wall, it is possible to discriminate between the intima and the media of the common carotid artery (Pignoli et all 1986).

\subsection{Aim and outline of the thesis}

\subsubsection{Aim of the thesis}

The aim of this thesis was to develop a method to detect early (atherosclerotic) changes in the structural and, hence, acoustical properties of the carotid artery wall. Deviations from the "normal" arterial wall, which may be different stages in atherogenesis, are characterized by changes in the composition and geometry inside the wall and do not necessarily influence the cross-sectional area of the lumen. Measurable changes in blood flow pattern are therefore not found. The mechanical properties of the arterial wall are influenced both by geometry (thickness) and tissue properties (elasticity) and it is not possible to discriminate between these influences from measurement of the mechanical behavior alone. Detailed information about changes in the thickness and composition (different types of tissue) of the arterial wall is important to study, for example, the natural history of atherogenesis. The difference in acoustical properties of different types of tissue may be used to determine the composition of the vessel wall. Assessment of the acoustical properties of the tissue in a thin layer (tissue characterization), however, is a major problem. The inhomogeneity of the sound field and radiation coupling effects cause major problems if broadband (conventional) pulses are used to characterize the tissue in a thin layer like the arterial wall. Therefore, in this thesis a method is introduced based on the use of narrowband ultrasound pulses at different emission frequencies: the NarrowBand MultiFrequency method (NBMF). The signal, obtained from deconvolution of the narrowband signals, is then a direct representation of the reflectivity of the investigated tissue at one of the different frequencies. Analysis of these signals may then give information about changes in acoustical properties and, hence, composition of the arterial wall.

\subsubsection{Outline of the thesis}

The mathematical models for ultrasound signals, used in this thesis are explained in chapter 2 . In these models, four influences on the received ultrasound 
signal can be distinguished:

-sound field model:

-attenuation model:

-transducer model:

-electrical input signal: diffraction and radiation coupling function attenuation function

emilted waveform

transducer impulse response function

broad-and narrowband excitation

Under certain conditions, it seems permitted to use a simplified model for ultrasound signals reflected by a layered tissue structure (arterial wall). These conditions depend on the characteristics of the attenuation and radiation coupling function and the bandwidth of the electrical input signal.

In the chapters 3 and 4 , the characteristics of the radiation coupling function will be investigated using numerical models for the sound field and the reflection at a tissue interface. To validate the sound field model and obtain realistic transducer parameters, a method to determine these transducer parameters from actually measured sound field pressure values is described in chapter 3. A numerical method to calculate the radiation coupling function of a tissue interface is validated by comparison with a well-known analytical solution for a simple transducer/reflector combination. The radiation coupling function for different reflector geometries in the sound field of a real transducer (chapter 3) will be calculated in chapter 4 . From the results it appears that measurement in the far field of a transducer is favourable to measurement in the near field.

Assuming a simplified model for ultrasound reflection, the received signal can be written as the convolution of a reflectivity function and some emitted waveform. Because broadband signals have a number of disadvantages (signal deformation, poor spectral SignaI to Noise Ratio), the use of narrowband signals at several distinct frequencies is suggested in chapter 5: the NarrowBand MultiFrequency method. To restore the loss in axial resolution caused by the use of narrowband pulses, the reflectivity function must be estimated by deconvolution of the received signal. If the impulse response functions, characterizing the acousto-electrical processes in the transducer, have a smooth broad band amplitude spectrum, simplification of the convolution model seems permitted: the electrical input signal can be used as a model for the emitted waveform (influence of transducer impulse response functions can be neglected). The use of Wiener-inverse filtering for deconvolving narrowband signals is investigated together with a method to reduce the arteficts resulting from the narrowband character of the emitted pulses.

Whether the performance of the narrowband multi-frequency method is degraded by neglecting the transducer impulse response functions is investigated in chapter 6. Computer simulated signals and real ultrasound signals are used. To determine the overall transducer impulse response, the use of cepstral filtering of actually received ultrasound signals is proposed in an appendix. 
The practical use of the narrowband multi-frequency method will be investigated in chapter 7 . An ultrasound system was modified so that it can emit and receive the waveforms as required in the narrowband multi-frequency method. Ultasound data from the carotid arteries of presumed healthy volunteers, some patients from the neurological clinic, and data from tissue mimicking phantoms will be processed according to the method suggested in chapters 5 and 6 .

The limitations and merits of the narrowband multi-frequency method will be discussed in chapter 8 . Recommendations for further research are presented.

\section{References}

Barnes R.W. Pittgers S.E. and Putney W.W. (1982): Real-time Doppler spectrum analysis: predictive value in defining operable carolid artery disease. Arch. Surg. $11752-57$

Blackshear W.M. Phillips D.J. Thiele B.L. Hirsch J.H. Chikos P.M. Marinelli M.R. and Ward K.J. (1979): Detection of carotid acclusive disease by ultrasonic imaging and pulsed Doppler spectrum analysis. Surgery $86 \quad 698-706$

Bom N. and J. Roelandt J. Eds. (1989): Intravascular ulirasound; Techniques, developments and clinical perspectiwes (Dordrecht: Kluwer)

Breslau P.J. (1982): Uttrasonic Duplex scanning in the evaluation of carotid artery disease. PhD. Thesis, University of Limburg, Maastricht

Brandestini M. (1978): A digital full range Doppler velocity meter. IEEE Tr. on Sonics and UItrasonics SU-25 287-293

Gross H.L. Ed. (1985): Atherosclerosis (Kalamazoo, Michigam: The Upjohn Company)

Green P.S. Taenzer J.C. Ramsey Jr. S.D. Holzemer F. Suarez J.R. Marich K.W. Evans T.C. Sandok B. A. and Greenleat J.F. (1977): A real time ultrasonic imaging system for carotid arteriography. Uitrasound in Med. \& Biol. 3 129-142

Hoeks A.P.G. (1982): On the development of a multi-gate pulsed Doppler system with serial data-processing. PhD. Thesis, University of Limburg, Maastricht

Hoeks A.P.G.Brands P.J.Smeets F.A.M. and Reneman R.S. (1990): Assessment of the distensibilty of superficial arteries. Ultrasound in Med. \& Biol. 16 121-128

Hoeks A.P.G.Reneman R.S and Peranneau P.A. (1981): A multigate pulsed Doppler system with seriall data processing. IEEE Tr. on Sonics and Uitrasonics SU-28 242-247

Hokanson D.E. Mozersky D.J. Summer D.S. and Strandness D.E. (1972): A phase lacked echotracking system tor recording arterial diameter changes in viwo. J. of App. Physiology $32 \quad 277-289$ Jones J.P. and Leeman S. (1984): Ultrasonic tissue characterization: a review. Acta Electronica 26 3-
31

Johnston K.W. Demorais D. Kassam M. and Brown P.M. (1981): Cerebrovascular assessment using a Doppler carotid scanner and realtime trequency analysis. J. Chin. Utrasound $9443-449$

Kuc $A$. and Schwartz M. (1979): Estimation the acoustic attenuation coefficient silope for liver from reflected ultrasound signals. IEEE Tr. on Sonics and Ultrasonics SU-26 353-362 Landini L. Picano E. and Sarnelii R. (1985): Ultrasonic energy base technique for characterizing
atherosclerosis. $J$. Biomed. Eng. 7 233-236 
Landini L. Sarnelli R. Picano E. and Salvadori M. (1986): Evaluation of frequency dependence of backscatter coefficient in normal and atherosclerotic aontic walls. Utrasound in Med. B Biol. $12 \quad 397$. 401

McDonald D.A. (1974): Blood flow in arteries. (Camelol Press LId., Southampton)

Peronneau P.A. Bournat J.P. Bugnon A. Barbet A. and Xhaard (1974): Theoretical and practical aspects of pulsed Doppler flowrnetry: real-ime application to the measure of instantaneous velocity profiles in vitro and in vivo. Cardiovascular applications of ultrasound Ed. Reneman R.S. (NorthHolland/American Elsevier, Amsterdam) 66-84

Pignoli P. Tremoli E. Poli A. Oreste P and Paoletti $A$ (1986): Intimall plus medial thickness of the arteriall wall: a direct measurement with ultrasound imaging. Circulation 74 1399-1406

Poli A. Tremoli E. Colombo A. Sirtori M. and Pignoli P. (1988): Ultrasonographic measurement of the common carotid artery wall thickness in hypercholesterolemic patients. Atherosclerosis $70 \quad 253-261$

Reneman R.S. and Spencer M.P. (1979): Local Doppler audio spectra in nomall and stenosed carotid arteries in man. Uitrasound in Med. \& Biol. 5 1-11

Reneman R.S. Van Merode T. Hick. P. and Hoeks A.P.G. (1985): Flow velocity patterns in and distensibility of the carotid antery bulb in subjects of various ages. Circulation $71500-509$

Reneman R.S. Van Merode T Hick P. Muytjens A.M.M. and Hoeks A.P.G. (1986): Age-related changes in carotid artery wall properties in men. Ultrasound in Med \& Biol. $12 \quad 465-471$

Sarnelli R. Landini L. and Squartini F. (1986): Alherosclerotic detection by ultrasounds. Appl. Path. 4 $270-275$

Stepanishem P.R. (1971): Transient radiation from piston is an infinite planar baffle. 4 . Acoust. Soc. Am. $49 \quad 1629-1638$

Van Baalen J.M. Jakimowicz J.J. and Reneman R.S. (1984): Noninvasive evaluation of carotid artery stenosis- comparison of direct and indirect techniques. Vasc. Surg. 18 88-95

Van Merode T. (1986): The use of a multi-gate pulsed Doppler syslem in the evaluation of the carotid artery circulation. PhD. Thesis, University of Limburg, Maastricht

Van Merode T. Hick P.J.J. Hoeks A.P.G. and Reneman IR.S. (1988): The diagnosis of minor to moderate atherosclerotic lesions in the carotid antery bifurcation by means of spectral broadening combined with the direct detection of llow disturbances using a multi-gale pulsed Doppler systern. Utrasound in Med. \& Biol. $14 \quad 459-464$

Van Merode T. Lodder J. Smeets F.A.M. Hoeks A.P.G and Reneman R.S. (1989): An accurate noninvasive method to diagnose minor atherosclerotic lesions in carotid artery bulb. Stroke 201336. 1340

Wells P.N.T. (1969): Physical principles of ultrasound diagnosis. (Academic Press, London and New York) 


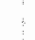

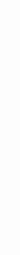


CHAPTER 2

ULTRASOUND SIGNALS 
Chapter 2 


\subsection{Introduction}

Characterization of tissue is generally performed by analyzing the signal backscattered from one or more tissue regions. Selection of tissue regions is achieved by multiplying the received signal $s(t)$ with a window function. The windowed signal then corresponds with a limited tissue region within the beam of the transducer. Signals returned by tissue interfaces (reflection) can also be used to assess certain acoustical properties of the tissue in between these interfaces.

To understand the problems associated with the analysis of backscattered and reflected signals, it is necessary to create mathematical models of these signals. With the use of these mathematical models, concepts, like diffraction and radiation coupling, will be elucidated.

\subsection{Mathematical model for ultrasound signals}

\subsubsection{Sample volume}

Assume that an infinitely short pulse, emitted at time $t=0$, is partly reflected by a scatterer at distance $z_{1}$ from the transducer. The reflection will arrive back at the transducer at time $t_{1}=2 z_{1} / c$. The speed of sound $c$ is assumed to be uniform in the medium between the transducer and the scatterer. Also, in this approach the dimensions of the transducer are assumed to be small in comparison with the distance between the scatterer and the transducer. Similarly, the reflection from a scatterer at distance $z_{2}\left(z_{2}>z_{1}\right)$ from the transducer will arrive at the transducer at time $t_{2}=2 z_{2} / c$. Selection of a part of the received signal with $t_{1}<t<t_{2}$ then corresponds to investigating the signals reflected by scatterers in a certain region in front of the transducer. This region is confined by two spheres with radius $z_{1}$ and $z_{2}$, and the dimensions of the ultrasound beam (see figure 2.1). Now, if the emitted pulse is not infinitely short, but has a duration $T_{p}$, the part of the pulse emitted at $T_{p}$, reflected by a scatterer at distance $z$, arrives at the transducer at $t=2$ $z / c+T_{p}$. It can easily be derived that the scatterer, of which the tail of the reflection arrives at $t=t_{1}$, is located at $z=z_{1}-T_{p} c / 2$. This means that the region from which the scatterers contribute to the signal within a time window becomes larger if the duration of the pulse increases. If the duration of the time window is $z_{\text {zero, }}$ the region from which the scatterers contribute to the ultrasound signal at a given moment in time is not infinitely small for a pulse with non-zero duration. This latter region is called the sample volume.

\subsubsection{Backscattered signals: diffraction function}

Scattering is the effect that part of the energy of an acoustic wave is directed in different directions if some inhomogeneity with a small dimension (compared to the wavelength) is encountered. One way to describe the backscattered signal returned by a tissue region is to assume a number of discrete scatterers at 
different locations in the sound field, each with its own scattering property. For a given transducer, the echo signal s(t) which returns from a number of $N$ scatterers can then be modeled as a series of convolutions (Hother and Bernatets 1984):

with:

$$
s(t)=e(t) * p_{t}(t) * p_{r}(t) * \sum_{i=1}^{N} h\left(z_{i}, t\right) * a\left(z_{i}, t\right) * u\left(z_{i}, t\right) * a\left(z_{i}, t\right) * h\left(z_{i}, t\right)
$$

s(i) measured ultrasound signal

e(t) electrical input signal

$p_{t}(t)$ electro-acoustical coupling function of transducer in transmission

$p_{r}(t)$ electro-acoustical coupling function of transducer in reception

$h\left(z_{i}, t\right)$ diffraction impulse response for a given transducer. From this function the particle velocity and pressure can be calculated if the emitted waveform is known (see chapter 3).

$a\left(z_{i}, t\right)$ function describing the attenuation

$u\left(z_{i}, t\right)$ function describing the backscaltering properties of the individual scatterers

$\mathbf{z}_{\mathrm{i}} \quad$ position of a scatterer

with bold symbols representing vectors and an * denoting the convolution operation.

$e(t), p_{t}(t)$ and $p_{r}(t)$ do not depend on the properties of the insonified medium and are replaced by a single expression $w(t)$ :

$$
w(t)=e(t) * p_{t}(t) * p_{r}(t)
$$

$p_{l}(t)$ and $p_{r}(t)$ are gathered in the overall transducer impulse response (system impulse response) $f(t)$ :

$$
f(t)=p_{t}(t) * P_{r}(t)
$$

$w(t)$ is, apart from a scale factor, the same as the signal received after reflection by a single perfect point scatterer in a non altenuating medium, using a point source transducer. Using equation 2.2 , equation 2.1 is written as:

$$
s(t)=w(t) * \sum_{i=1}^{N} h\left(z_{i}, t\right) * a\left(x_{i}, t\right) * u\left(z_{i}, t\right) * a\left(z_{i}, t\right) * h\left(z_{i}, t\right)
$$

The diffraction and attenuation terms occur twice because the waves travel from the transducer to the scatterer and back again. Strictly speaking, combination of the frequency dependent attenuation and diffraction impulse response, like in 

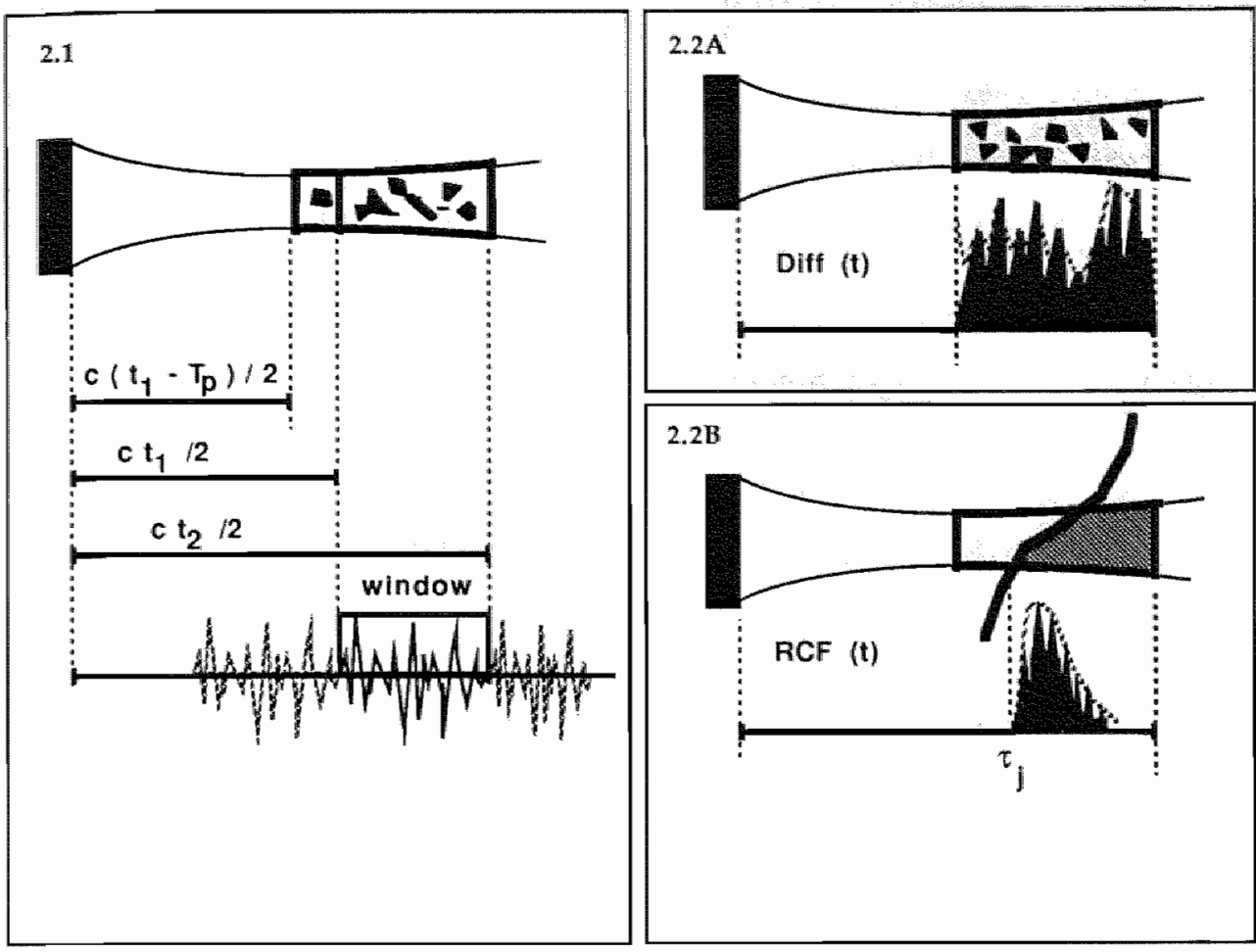

figure 2.1 The region from where the echo signal in time window $t_{1}<t<t_{2}$ originates from if the duration of the pulse is $t p$ is indicated with thick black lines. The left part in thick lines is present even it $t_{2}=11$. This region is called the sample volume.

figure 2.2 The impulse response of a region of scatterers (Diff( $(z, 1)$, top) in comparison with the impulse response (RCF (t), bottom) of a smooth surface.

equation 2.1, is incorrect. In the calculation of the diffraction impulse response function, one must either assume the attenuation to be zero, or the attenuation and diffraction must be combined in the calculation of a single diffraction/attenuation function. The reason for this is that the attenuation of a pulse originating from a point on the transducer surface depends on the distance traveled. However, the differences in pathlength, which cause the diffraction effect, are on the order of a few wavelengths. In biological tissues, the difference in attenuation due to such small differences in distance are negligible (frequencies $<20 \mathrm{MHz}$ ). Therefore, equation 2.1 is a good model for backscattered ultrasound signals used in diagnostic ultrasound. 
In the frequency domain equation 2.4 becomes:

$$
S(f)=W(f) \sum_{i=1}^{N} H\left(z_{i}, f\right) A\left(z_{i}, f\right) \cup\left(z_{i}, f\right) A\left(z_{i}, f\right) H\left(z_{i}, f\right)
$$

where capitals are used to indicate the Fourier transformed time signals.

If the signal is investigated within a short time window $\left(t_{1}<t<t_{2}\right)$, it is reasonable to approximate the attenuation function by a constant function $a\left(t, t_{2}, t\right)$, which represents the average attenuation for that region of interest. The signal windowed between $t_{1}$ and $t_{2}$ is written as $s\left(t_{1}, t_{2}, t\right)$. The attenuation terms can then be brought outside the summation:

$$
s\left(t_{1}, t_{2}, t\right)=w(t) * a\left(t_{1}, t_{2}, t\right) * a\left(t_{1}, t_{2}, t\right) * \sum_{j=1}^{M} h\left(z_{j}, t\right) * h\left(z_{j}, t\right) * u\left(z_{j}, t\right)
$$

It is remarked that the summation term has changed because only scatterers contributing to the signal within the time window are taken into account. For each time window, the expression with the summation sign must be evaluated separately. Here, this expression is called the diffraction function:

$$
\operatorname{Diff}(t, t, t)=\sum_{j=1}^{M} h\left(z_{j}, t\right) * h\left(z_{j}, t\right) * u\left(z_{j}, t\right)
$$

For each individual scatterer and for commonly used transducers, $h\left(z_{j}, t\right) * h\left(z_{j}, t\right) * u\left(z_{j}, t\right)$ is a function with a very short duration. Because of the discontinuous spacing between the scatterers, the summation of these functions results in a noise-like diffraction function $\operatorname{Diff}\left(t_{1}, t_{2}, t\right)$ (figure 2.2, upper part). Because the choice of a time window corresponds to a given tissue volume, the dependence of the diffraction function on the choice of the window will be expressed as:

$$
\operatorname{Diff}\left(t_{1}, t, t\right)=\operatorname{Diff}(\zeta, t)
$$

where $\zeta$ is the distance between the transducer and the center of the tissue volume which is determined by the choice of $t_{1}$ and $t_{2}$. The two attenuation terms in equation 2.6 are combined in a single function a $\left(\zeta_{,} t\right)$. Using a similar notation for the other signals depending on the choice of the window, equation 2.6 is then expressed as:

$$
s(\zeta, t)=w(t) * a(\zeta, t) * \operatorname{Diff}(\zeta, t)
$$

where $a(\zeta, t)$ is the roundtrip average attenuation. 

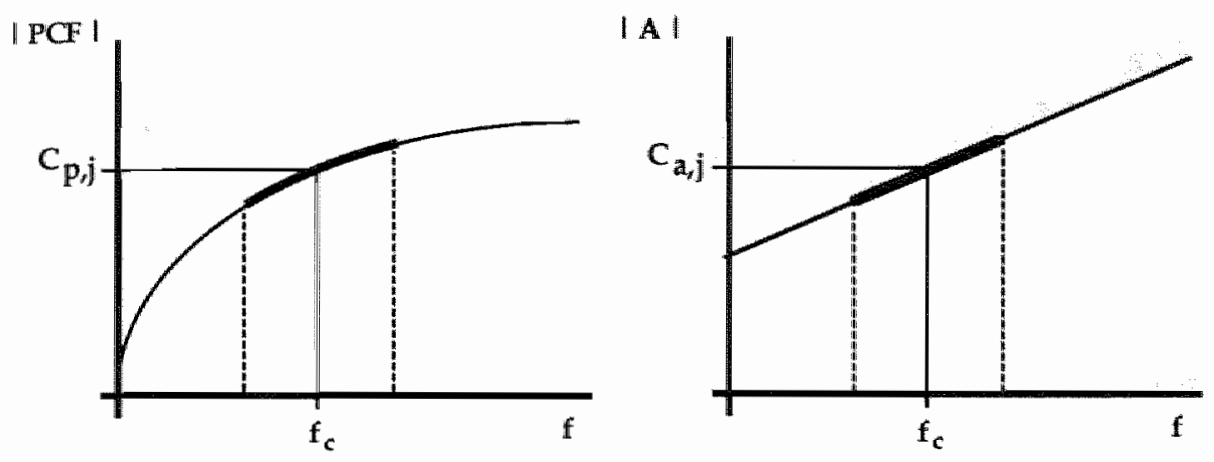

figure 2.3 Determination of $\mathrm{C}_{\mathrm{p}, \mathrm{j}}$ and $\mathrm{C}_{\mathrm{a}, \mathrm{j}}$ from the amplitude spectra of the Pressure Coupling Function ( $\mathrm{PCF}(\mathrm{f})$ ) and attenuation function ( $\mathrm{A}(\mathrm{t}))$, respectively, at center frequency $\mathrm{I}_{\mathrm{C}}$ and a bandwidth as indicated by vertical dotted lines.

\subsubsection{Reflected signals: Radiation Coupling Function}

Reflection occurs at the boundaries of tissue regions (with dimensions much larger than the wavelength of the ultrasound) with different acoustical impedances. The reflection from a boundary surface between two tissue regions is written in a way similar to equation 2.1. By regarding the surface as an infinite number of small, adjacent scatterers, the summation term in equation 2.5 transforms to an integration over the reflecting surface. Evaluation of this integral results in the Radiation Coupling Function $(R C F(t))$. The expression for the signal received from a single surface then becomes:

$$
s(t)=w(t) * a(\zeta, t) * R C F(t)
$$

In literature on the subject, the influence of the radiation coupling effect on the received ultrasound signal is generally expressed in terms of the Pressure Coupling function. The Pressure Coupling Function, PCF(t), is the time derivative of the radiation coupling function, multiplied by the mass density $\rho$ of the medium (Cassereau and Guyomar 1988, Rhyne 1977). Equation 2.10 is then written as:

$$
s(t)=w^{\prime}(t) * a(\zeta, t) * P C F(t)
$$

where $w(t)=p \frac{d w^{\prime}(t)}{d t}$.

Here again, the attenuation is approximated by a constant function where $\zeta$ is the distance between the transducer and the reflector. It should be noted that the pressure coupling function depends on both the sound field (transducer and medium in between) and the reflecting properties and shape of the interface 
(acoustical properties of the tissues on eilther side, geometrical shape and surface roughness).

For a continuous reflecting surface the distance between different points at the surface (i.e. infinitesimally small scatterers) and the transducer is a smooth function. Summation (integration) of the reflections from these points results in a smooth continuous function (figure 2.2 lower part). It should be well noted that the pressure coupling function must be evalluated for every transducer/reflector combination and for every position of the reflector in the sound field.

\section{2,2.4 Ultrasound signals from layered structures: vessel walls}

For frequencies below $10 \mathrm{MH} z$, the signal returned by a laminar structure like a vessel wall is dominated by the reflections of the interfaces between the different layers. The signal can therefore be written as the summation of a number of $\mathrm{N}$ individual reflections:

where

$$
s(t)=\sum_{j=1}^{N} \operatorname{ref}(t)
$$

$$
\operatorname{ref}_{j}(t)=w^{\prime}(t) * a_{j}(t) * \mathbb{P C F} j(t)
$$

ref $_{j}(t)$ is the signal that would be obtained if only the $j$-th reflecting surface is present in the sound field of the transducer (see equation 2.11). For notational simplicity, the accent of $w^{*}(t)$ in equation 2.13 will be omitted in the following. The attenuation of the forward and backward traveling pulses are gathered in an average attenuation function $\mathrm{a}_{\mathrm{j}}(\mathrm{t})$. PCF $\mathrm{PC}(\mathrm{t})$ is the pressure coupling function for the $j$-th tissue interface. If the reflection coefficient $C_{r, j}$ (the $r$ denotes "reflection") is uniform on the whole surface, $\mathrm{PCF}_{j}(\mathrm{t})$ is written as:

$$
P C F ;(t)=C_{r, j} P C F_{j}(t)
$$

If the different reflections do not overlap, it is possible to select a reflection from the received signal and calculate its spectrum:

$$
\operatorname{Ref}_{j}(f)=W(f) A_{j}(f) C_{r, j} P C F_{j}(\rho)
$$

Generally, the amplitude spectra of the pressure coupling functions of different reflectors $\left(\mathrm{PCF}_{\mathrm{j}}(\mathrm{f})\right)$ are not the same, but they can often be considered flat in a given frequency range. The attenuation function is also a smooth function of frequency. If $w(t)$ is a narrowband signal (see section 2.5), the behavior of $A_{j}(f)$ and $P_{C F}(f)$ is important only in a small frequency range. Within such a small frequency range (use of narrowband signals) the attenuation and $P C F(\cap)$ will be approximated by al constant function (see figure 2.3). Equation 2.15 then becomes:

$$
\operatorname{Ref}_{j}(\theta)=W(f) C_{r, j} C_{p, j} C_{a, j}
$$



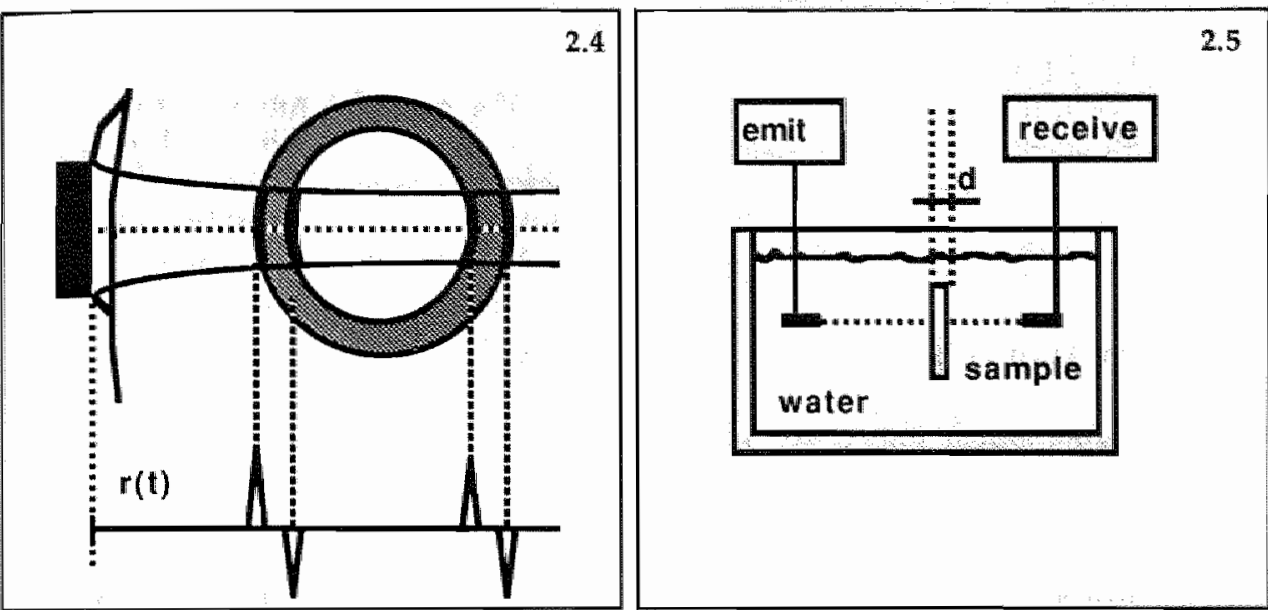

figure 2.4 Reflectivity function resulting from a number of ideal reflectors (uniform PCF).

figure 2.5 Determination of the attenuation of a piece of materiall (thickness $d$ ) in a water tank. The emitter and receiver circuils are indicated in the figure.

where $C_{p, j}$ and $C_{a, j}$ are the average values of the pressure coupling function and the attenuation function of the $j-t h$ reflector, respectively. These three constants are written as one constant $C_{j}$. The radiation coupling function of a reflector is zero until certain time $\tau_{j}$ which is the time corresponding to the shortest distance between the transducer and the reflecting surface (see figure 2.2). Because the pressure coupling function is the time derivative of the radiation coupling function, the first one is also zero until that time. Using equations 2.12 and 2.16 , the signal received from a number of $N$ reflectors is then written as:

$$
s(t)=w(t) *\left[\sum_{j=1}^{N} C_{j} * \delta\left(t-\tau_{j}\right)\right]
$$

The $\delta$-function accounts for the delay due to the travel time for a pulse to travel between the transducer and back. The term in square brackets will be called the reflectivity function $r(t)$.

$$
s(t)=w(t) * r(t)
$$

The reflectivity function for a number of ideal reflectors (tissue interfaces), like in figure 2.4, is depicted in the same figure. The spikes ( $\delta$-functions with amplitude $C_{j}$ ) correspond to the position of the reflectors and the amplitude of the spikes is determined by the attenuation of the waveform, reflected by that 
tissue interface, and by the pressure coupling function in the frequency bandwidth of the pulse. The conditions for which the attenuation and the pressure coupling functions can be regarded as a constant function in the frequency domain must be investigated.

\subsection{Differentiation of tissues}

\subsubsection{Possibilities for differentiation of tissues}

In the mathematical model for ultrasound presented in the previous sections, there are 3 components that depend on the acoustical properties of the insonified tissues. These components are:

1- The diffraction function Diff $(\zeta, t)$. This function depends on the backscattering properties $u(x, t)$ of the individual scatterers, but also on the spatial distribution of the scatterers that contribute to the signal in the time window.

2- The attenuation function $A(\zeta, t)$ which represents the average roundtrip attenuation between the transducer and the tissue region or the reflecting surface.

3- The Pressure Coupling Function PCF(t) of a reflecting surface depends on the reflection coefficient. The reflection coeffient of a tissue interface depends on the acoustical impedance of the tissues on either side.

All three components offer possibilities for differentiation of tissues.

\subsubsection{Backscatter analysis}

Models for backscattered signals are based on certain assumptions concerning the reflectivity (determined by shape and size) and the distribution of the scatterers (e.g. density) (Lizzi et al 1983 and 1986, Chivers 1977, Wagner et al 1988). From these models, Diff $(\zeta, 1)$ can be calculated. Assuming a frequency independent sound velocity, the wavelength $\lambda$ of the ultrasound signal is inversely proportional to frequency (equation 1.2). Summation of the backscattered waves from the different scatterers at the transducer surface (equation 2.7) then results in interference phenomena. The degree of destructive or constructive interference depends on the distribution of the interdistance between the scatterers in relation to the wavelength. The frequency dependent behavior of the backscatter signal is therefore related to the texture of the tissue and some investigators have developed methods to derive texture parameters from backscattered signals (Insana and Hall 1990a and 1990b, Insana et al 1990, Lizzi et all 1983 and 1986, Wagner et al 1988). A good example of the relation between backscatter and the structure of the tissue was recently demonstrated by De Kroon et al (1991), who found that the backscattering strength of vessel wall tissue depends on the angle between the orientation of the fibers in the tissue and the direction of the sound beam. 
The speckle pattern observed in B-mode images is also caused by the interference phenomena which determine the diffraction function. Because the envelope information of the B-mode image is readily available on most commercial ultrasound scanners, some investigators have tried to use certain statistical characteristics of the observed speckle patterns for tissue characterization (Jones and Kimme-Smith 1982, Kimme-Smith and Jones 1984). Only pathological changes which result in very large changes in the structure of organs can be found from the analysis of speckle patterns.

\subsubsection{Attenuation}

\subsubsection{Definition}

Where the analysis of backscattering properties of media is related to the local structure of the tissue, attenuation is a typical bulk parameter. It describes how the amplitude of a plane acoustic wave decreases when traveling through a biological material. This decrease in amplitude is characterized by the attenuation coefficient $\gamma\left[\mathrm{m}^{-1}\right]$ introduced in chapter 1 and defined below. If $\mathrm{Amp}_{0}(\mathrm{f})$ is the amplitude spectrum of a pulse at some reference position $\zeta=0$ (e.g. transducer surface), the amplitude spectrum of the pulse after traveling a distance $d_{1}$ will be expressed as:

$$
\operatorname{Amp}\left(d_{1}, f\right)=\operatorname{Amp}_{0}(f) \exp \left[-\gamma(f) d_{1}\right]
$$

The attenuation function $A(d, f)$ is then:

$$
\mathrm{A}(\mathrm{d}, \mathrm{f})=\exp [-\gamma(\mathrm{f}) \mathrm{d}]
$$

The value of $\gamma$ is generally frequency dependent:

$$
\gamma(f)=a_{0}+a_{1} f^{n}
$$

where $\mathrm{a}_{0}\left[\mathrm{~m}^{-1}\right]$ and $\mathrm{a}_{1}\left[\mathrm{~m}^{-1} \mathrm{~S}^{\mathrm{n}}\right]$ are constants.

In the formulation above, $\gamma$ is used to describe the overall attenuation and the power $\mathrm{n}$ is used to allow for nonlinear dependency on frequency. Velocity dispersion is ignored, which means that the attenuation is purely an amplitude modulating effect. There are two sources for ultrasonic attenuation. First, part of the acoustical energy is absorbed by chemical and viscous relaxation processes. The second cause for loss of acoustical energy in the direction of the beam is the scattering of acoustical waves in other directions.

The reason why attenuation and the frequency dependence of attenuation can be used to discriminate between tissue types is that the relaxation processes, which characterize most sources of attenuation (Jongen et al 1986), depend on the type of macromolecules present in the different types of tissue. Tuthill and associates 


\begin{tabular}{|l|l|l|}
\hline & $\begin{array}{l}\text { at, attenuation at } \\
1 \mathrm{MHz}\left[\mathrm{dB} \mathrm{cm}^{-1}\right]\end{array}$ & $\begin{array}{l}\text { frequency dependence } \\
n-1\end{array}$ \\
\hline water & 0.0022 & 2 \\
blood & 0.18 & 1.3 \\
brain & 0.85 & -1 \\
iat & 0.63 & -1 \\
muscle & $1.3 \cdot 3.3$ & 2 \\
skull bone & 13 & -1 \\
\hline lung & 41 & \\
\hline
\end{tabular}

table 2.1 Acoustic aftenuation parameters for different types of tissues (from Jones and Leeman 1984): $(1)=a_{1} f^{n}$

(1989), for example, showed the attenuation in the liver to be dependent on glycogen content. It was generally found that acoustic attenuation is enhanced with increased content of structural proteins (e.g. collagen), while in case of an increased water content it is lower (Jones and Leeman 1984).

\subsubsection{Measurement of attenuation in-vitro}

The most accurate way to determine the attenuation of a piece of material is the transmission technique. This technique is presented in figure 2.5. A specimen (sample) of the material with known thickness $d$ is placed between two transducers. The surrounding medium (often water) has a very low attenuation in comparison with the tissue material. The emitting transducer emits long almost continuous wave pulses at different frequencies. At each frequency, the amplitude received at the opposite transducer with and without the specimen in between are then compared. From this comparison the frequency dependence of the attenuation is evaluated by fitting an attenuation law model to the measured data (Hottier and Bernatets 1984). In a similar experimental set-up the second transducer is replaced by a plane reflector. The frequency dependence of the attenuation can then be assessed by comparing the spectrum of the reffected pulses with and without the specimen in between. Because the signals are measured in the same region of the sound field, there is no need for correction of diffraction effects (see section 2.4).

Some values for tissues measured with transmission or plane reflector techniques are presented in table 2.1. The data are from Jones and Leeman (1984). A large compilation of acoustic properties of different tissues is found in a paper by Goss et al (1978). Landini and associates found that different types of atherosclerotic lesions show significant changes in attenuation and backscattering coefficients in comparison with normal arterial wall tissue (Sarnelli et al 1986, Landini et al 1986). Although these in-vitro measurement methods are very accurate, the results are not necessarily representative of attenuation values in living tissues. Although changes occur when tissue is 
removed from the living body, these data indicate that attenuation values do depend on tissue type and pathology.

\subsubsection{Measurement of attenuation in-vivo}

Although transmission experiments provide the most accurate way to determine acoustic attenuation, this method is not applicable to most parts of the human body. The dimensions of the human body are so large that no measurable signal arrives at the opposite transducer. Further, the presence of bony structures or gas filled organs (lungs, intestines) result in a great loss in acoustic energy due to reflection and attenuation. Therefore, in most organs attenuation parameters must be derived from reflected (backscattered) ultrasound signals. In this context ultrasound imaging that uses the transducer both for emission and reception is generally called reflection mode imaging.

In reflection mode imaging, ultrasound attenuation can be measured by comparing the signals from two regions along the same A-line, which are some distance apart. The attenuation as a function of rrequency is then found by comparing the power spectrum of the signals in the two regions (see figure 2.6). The processing of the windowed signals is elucidated in section 2.3.3.4.

Several methods have been developed to establish the frequency dependence of the attenuation. Basically the difference between the methods is the selection of the regions from which the spectra are compared. Ophir and coworkers used a number of adjacent A-lines and analyzed the difference between the spectra in two fixed windows (figure 2.7a) (Maklad et al 1984, Ophir and Maklad 1978). Kuc et al compared the signal spectra from a pair of windows at a fixed distance. By shifting the begin of the first window allong the received signal a large number of attenuation functions was averaged (figure 2.7b) (Kuc 1984, Kuc and Schwartz 1979). If a linear dependence on frequency $(n=1$ in equation 2.21) and a Gaussian shaped spectrum of the pulse is assumed, a relationship can be calculated between the center frequency and depth. Fink and colleagues (1984 b) proposed the use of short-time Fourier analysis in a sliding window along an A-line (figure $2.7 \mathrm{c}$ ). The center frequency of the signal in the window was then plotted as a function of depth. The decay in center frequency per unit of distance is then used to differentiate between different types of tissue.

\subsubsection{Aftenuation "in a layered tissue (vessel walls)}

To measure the attenuation by a thin tissue layer (e.g. vessel wall) one has to use the reflections from the boundaries on both sides of the layer, or one has to compare the backscatter spectra of the tissue regions just in front and just after the tissue layer(s) (figure 2.6). Likewise, the attenuation in large homogeneous organs is also assessed by comparison of the backscatter signals from different tissue regions (see previous section). The reflections of the boundaries can only be used for spectral analysis, if they are clearly separated and not disturbed by backscatter signals from the tissue on both sides of the boundaries. 

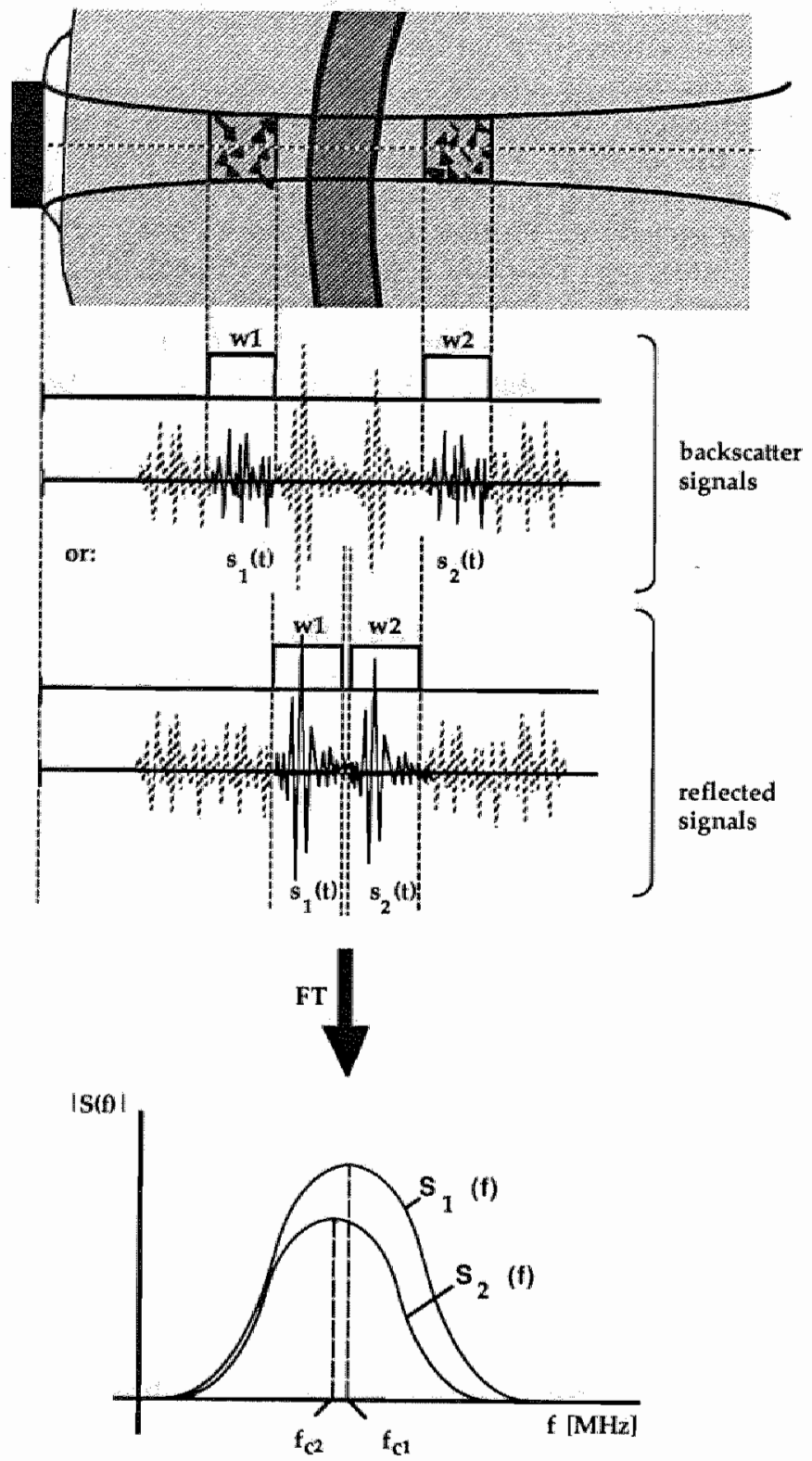

figure 2.6 The signals originating from different tissue regions along the ultrasound beam are selected by windowing. Comparison of the spectra allows determination of the attenuation of the tissue in between the two regions. 

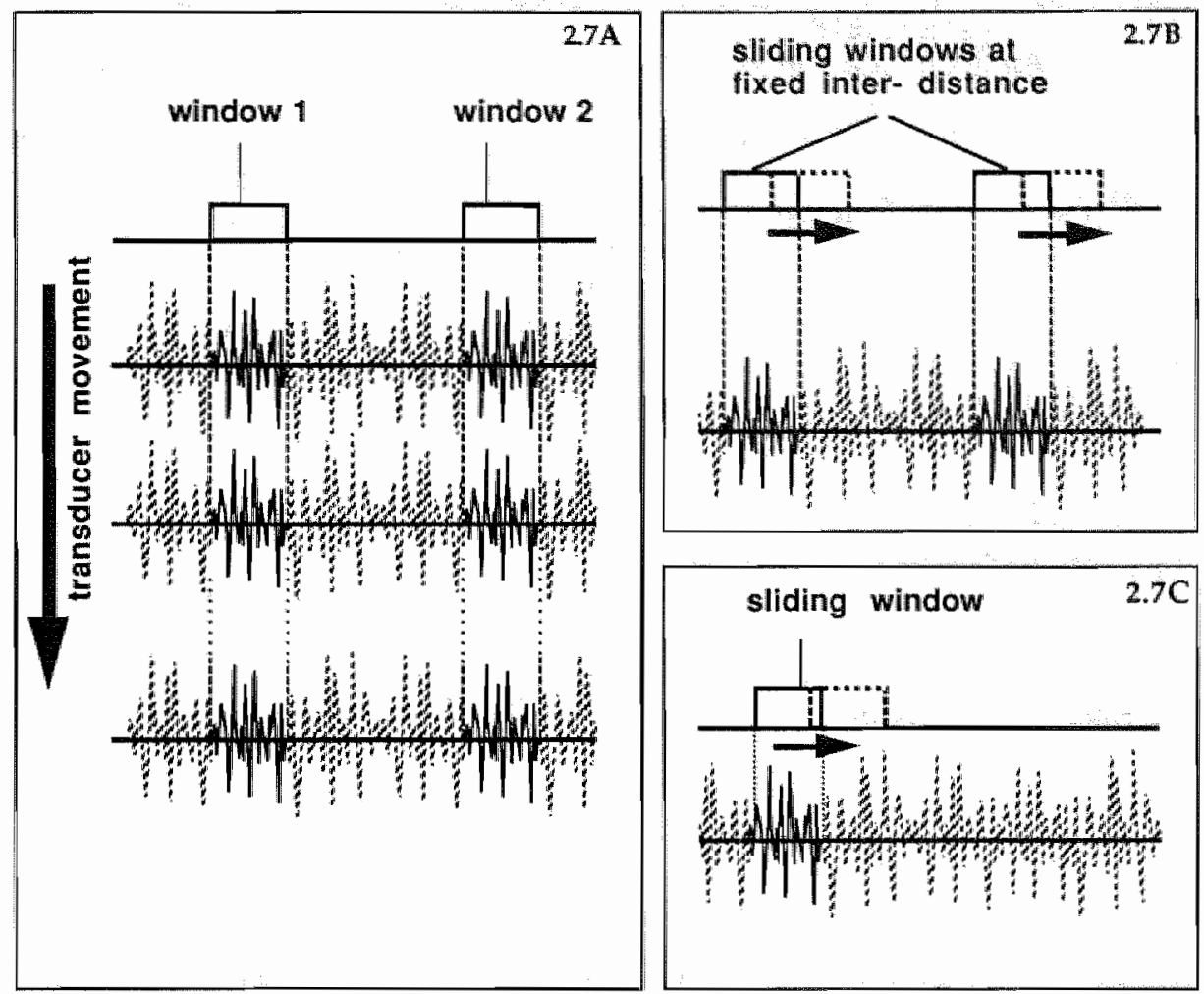

figure 2.7 Different methods of comparing short segments of ultrasound data.

2.7a: The transducer is moved in a certain plane. The received ultrasound signals of two fixed time windows are evaluated for every transducer position.

2.7b: The signall from two windows, which are a fixed distance apart from each other, are evaluated for different positions of the lirst window.

2.7c: The variation of the spectrum of the signal in a sliding time window is investigatied.

The spectrum of the backscattered signals from different tissue regions (see figure 2.6) is expressed as (see equation 2.9):

$$
\begin{aligned}
& S_{w 1}(f)=W(f) A_{1}(f) \operatorname{Diff}_{1}(f) \\
& S_{w 2}(f)=W(f) A_{2}(f) \operatorname{Diff}_{2}(f)
\end{aligned}
$$

$S_{w j}(f), A_{j}(f)$ and $D_{i f f}(t)$ are short notations for the spectrum of $s(t)$ in the time windows $w_{j}$, the attenuation function between the transducer and the $j$-th tissue region and the diffraction function of the j-th tissue region, respectively. 
Equivalent expressions are found if $s_{w 1}(t)$ and $s_{w 2}(t)$ are reflections from two tissue interfaces (see equation 2.12):

$$
\begin{aligned}
& S_{w 1}(f)=\operatorname{Ref}_{1}(f)=W^{\prime}(f) A_{1}(f) \operatorname{PCF}_{1}(f) \\
& S_{w 2}(f)=\operatorname{Ref}_{2}(f)=W^{\prime}(f) A_{2}(f) \operatorname{PCF}_{2}(f)
\end{aligned}
$$

Here, the pressure coupling functions of the different interfaces are denoted by $\mathrm{PCF}_{1}(\mathrm{t})$ and $\mathrm{PCF}_{2}(\mathrm{t})$. Again, the attenuation terms for the forward and backward traveling waves are incorporated in a single attenuation function. If the pressure coupling functions (or the diffraction functions) are the same, it is possible to evaluate the attenuation of the tissue in between the tissue interfaces by comparison of the two spectra:

$$
\log \left(\frac{A_{2}(f)}{A_{1}(f)}\right)=\log \left\{\frac{S_{2}(f)}{S_{1}(f)}\right\}
$$

With an exponential attenuation law, like equation 2.18 , and $\left(\mathrm{d}_{2}-\mathrm{d}_{1}\right)$ being the "distance" between the two reflecting tissue layers lor backscattering tissue regions), it follows that (accounting for the round-trip path of a pulse between the interfaces, or the tissue regions, Hottier and Bernatets 1984):

$$
\gamma(\mathrm{l})=\frac{-1}{2\left(\mathrm{~d}_{2}-\mathrm{d}_{1}\right)} \log \left(\frac{\mathrm{A}_{2}(\mathrm{f})}{\mathrm{A}_{1}(\mathrm{f})}\right)
$$

Assuming an attenuation model like in equation 2.21, the parameters can then be found from curve fitting. The derivation given above only holds if the pressure coupling functions or the diffraction functions in the two expressions for the received signals are the same. If they are not, it is necessary to compensate for the influence of these functions. This aspect will be dealt with in section 2.4.

\subsection{Influence of Diffraction and Radiation Coupling}

Especially for broad band signals, the assumption that the diffraction function of two different tissue regions is the same is generally not valid. Analogously, the pressure coupling functions of two different reflectors will hardly be the same in a broad frequency range. To assess the attenuation, according to the derivation given in section 2.3.3.4, it is necessary to compensate for the difference in pressure coupling functions between the surfaces $\left(\mathrm{PCF}_{1}(f)\right.$ vs. $\mathrm{PCF}_{2}(\mathrm{f})$ ) or, for backscattered signals, for the difference in diffraction function between both regions (Diff ${ }_{1}(f)$ vs. Diff $f_{2}(f)$. Equation 2.26 is then replaced by:

or:

$$
\log \left\{\frac{A_{2}(f)}{A_{1}(f)}\right)=\log \left(\frac{S_{2}(f)}{S_{1}(f)} \frac{\operatorname{PCF}_{1}(f)}{\operatorname{PCF}_{2}(f)}\right)
$$

$$
\log \left(\frac{A_{2}(f)}{A_{1}(f)}\right)=\log \left(\frac{S_{2}(f)}{S_{1}(f)} \frac{D_{i f f}(f)}{\operatorname{Diff}_{2}(f)}\right)
$$


Much literature has appeared on the influence of the diffraction effect and the possibilities for correcting the spectra from different tissue regions (O'Donnel 1983, Fink and Cardoso 1984, Fink et al 1984a and 1984b, Verhoef et al 1984, Laugier et al 1987). The concept of most methods is to use signal spectra obtained from a plane reflector placed at different distances from the transducer or spectra of backscatter signals from tissue mimicking phantoms, placed at different depths. One of the spectra (generally the one from the focal distance of the transducer) is taken as the reference spectrum $\operatorname{Diff}_{2}(f)$ in equation 2.28. For each distance this reference spectrum is then divided by the local spectrum. In this way for each distance (time window) a diffraction correction filter is obtained. Multiplication of the measured spectrum at different depths with the diffraction correction filter results in a spectral estimate from which much of the diffraction error is eliminated. Although the variance in attenuation parameter estimates decreases when these methods are used, none of the methods is mathematically or physically correct. Ophir introduced a method which avoids the use of diffraction correction filters (Ophir and Mehta 1988). Most recently, the use of non-diffracting transducers was suggested by Greenleaf and associates (Lu and Greenleaf 1990). The pressure distribution in a plane perpendicular to the axis of the sound beam of a non-diffracting transducer does not change over a long distance range. All these methods were developed for large tissue regions with constant acoustic properties.

Because most tissue characterization methods known from literature are based on analysis of backscattered signals, the pressure coupling function of tissue interfaces was never a major topic of interest. Cassereau and Guyomar (1988) and Rhyne (1977) investigated the pressure coupling function of smooth and that surfaces, respectively. If it is possible to calculate the influence of the geometry of the reflecting tissue interface and the position in the sound field on the pressure coupling function, some first order correction according to equation 2.29 may be possible. The morphology of the interface (rough or smooth) and the shape (geometry) also influence the pressure coupling function (Chivers and Santosa 1986), but these effects are difficult to take into account.

\subsection{Conventional (broadband) and narrowband ultrasound}

As explained in section 2.1, the sample volume depends on the duration of the pulse. In order to be able to discern two adjacent reflectors from the amplitude of the received echo signal, the duration of the pulse is generally chosen as short as possible. In the frequency domain such a short pulse has a relatively broad spectrum and is therefore called a broadband pulse. Narrowband pulses have a very narrow frequency spectrum (see figure 2.8). Broadband pulses have several disadvantages if reflections from tissue interfaces are to be used for tissue characterization. Firstly, although the reflections may be well separated, it is in general not possible to select the individual reflections from a noisy backscattered signal without making windowing-errors (truncation of the reflections at different places). Secondly, the spectral SNR and resolution of short data segments are very poor. Thirdly, the assumption that the pressure coupling function and the attenuation function can be approximated by constants is 
generally not walid if the frequency range of the input signal is very wide: the simple signal model according to equation 2.17 is not valid.

Narrowband signals have a higher spectral SNR than broadband signals if the power of the signal in the time domain is the same. Narrowband signals also have some disadvantages. Firstly, the duration of a signal in time is inversely proportional to the bandwidth in the frequency domain. Narrowband signals therefore have a long duration, which results in a poor temporal resolution: reflections from closely spaced tissue interfaces will overlap. The resolution must be restored by deconvolution. Secondly, because emission of a narrowband pulse will give information only within its own narrow frequency band, the spectral information about the attenuation function, for example, must then be obtained by using different emission frequencies. A great advantage of narrowband signals, however, is that the signal model according to equation 2.18 is valid if the pulse is sufficiently narrowbanded.

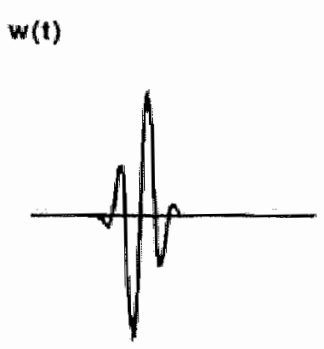

$w(t)$

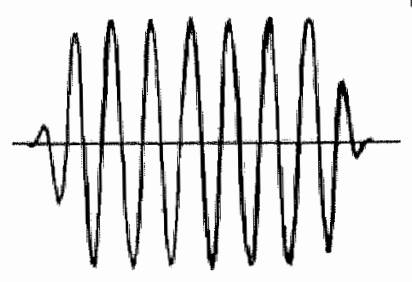

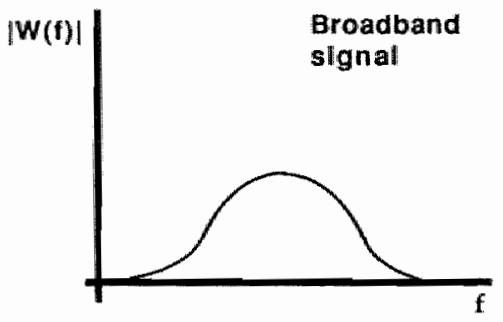

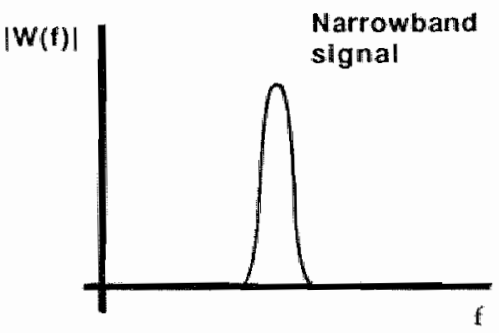

figure 2.8 Broadband signal (upper panels) and a narrowband signal (lower panels) and their amplitude specira.

\subsection{Vessel wall tissue characterization}

There are different approaches to characterize the acoustical properties of the arterial wall. Assuming the attenuation of the blood to be negligible when compared to tissue (Goss 1978), the attenuation caused by the vessel wall can be calculated from the integrated power in the regions corresponding with the anterior and posterior walls (regions 2 and 4 in figure 2.9). 
If the tissue in front of the artery and the tissue at the back are the same, another possibility is to calculate the power for each frequency in regions just in front and just behind the vessel walls (regions 1 and 5 in figure 2.9). If these regions do not contain specular reflections, this method may be less susceptible to variations in reflectivity due to the orientation of the transducer. However, the situation that there are absolutely no specular reflectors in both regions is likely to be rare.

The acoustic properties of blood are not expected to vary drastically from person to person. Therefore, a third method is to compare of signals in one of the wall regions ( 2 and 4 ) with the signal returned by the blood in the lumen (region 3 ). The spectrum of the signals originating from the lumen are then used as a reference.

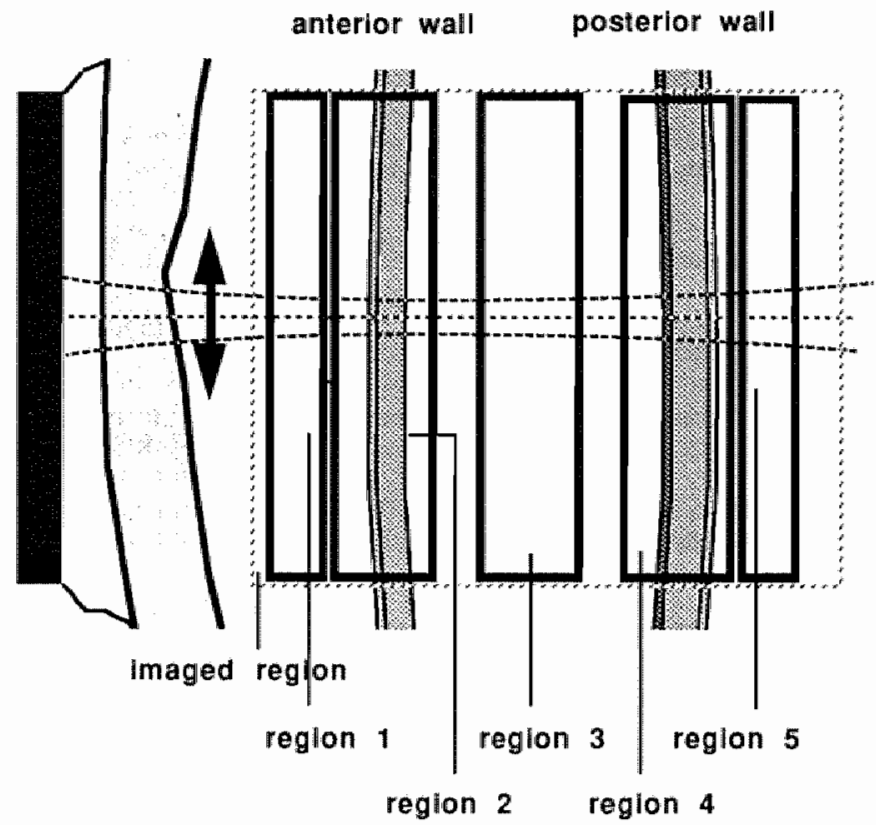

figure 2.9 Schematic presentation of a blood vessel, investigated with a linear array Iransducer. The imaged region is indicated as well as different regions that can be used for tissue characterization. 


\section{References}

Cassereau D. and Guyomar D. (1988): Computation of the impulse diffraction of any obstacle by impulse ray madelting - Prediction of the signal distortion. $d x$, Acoust. Soc. Amer. 84 1504-1516

Chivers F.C. (1977): The scattering of ultrasound by human tissues - some theoretical models. utrasound in Med. Biol. 3 1-13

Chivers R.C. and Santosa F. (1986): Numerical considerations for interface reflections in medical ullirasound. Physs. Med. Biol. 31 19-37

Fink M.A. and Cardoso J.-F. (1984): Diffraction effects in pulse-echo measurement. IEEE Trans. Sonics and Uitrason. SU-31 313

Fink M. Hottier F. and Cardoso J..F. (1984a): Ultrasonic signal processing for in wivo attenuation measurement: short time Fourier analysis. Uitrasonic Imaging 5 117-135.

Fink M. Cardoso J.F. and Laugier P. (198:4b): Diffraction effect analysis in medical echography. Acta Electronica $26 \quad 59-80$

Goss S.A. Johnston R.L. and Dunn F. (197B): Comprehensive compilation of empirical ultrasonic propenties of mammalian tissues. J. Acoust, Soc. Am. 64 423-457

Hottier F. and Bernatets J.L.L. (1984): Estimation of ultrasonic attenuation in biological tissues. Acta Electronica $2633-58$

Insana M.F. and Hall T.J (1990a): Characterizing the microstructure of random media using ultrasound. Phys. Med. \& Biol. 35 1373-1386

Insana M.F. and Hall T.J.(1990b): Parametric ultrasound imaging from backscatter coefficient measurements: image formation and interpretation. Uitrasonic Imaging 12245

Insana M.F. Wagner R.F. Brown D.G. and Hall T.J. (1990): Describing small-scale structure in random media using pulse-echo ultrasound. J. Acoust. Soc. Am. $87179-192$

Jones J.P. and Kimme-Smith C. (1982): An analysis of the parameters affecting texture in a B-mode ultrasonogram. Acoustical /maging 10 295-308

Jones J.P. and Leeman S. (1984): Utrasonic tissue characterization: a review. Acta Electronica 26 331

Jongen H.A.H. Thijssen J.M. Vant den Aarssen M. and Verhoel W.A. (1986): A general model for the absorplion of uttrasound by biological tissues and experimental verification. J. Acoust. Soc. Am. 79 $535-540$

Kimme-Smith C. and Jones $\downarrow$.P. (1984): The relative effects of system parameters on texture in grayscale ultrasonograms. Uttrasound in Med. \& Biol. 10 299-307

De Kroon M.G.M. Van der Wal L.F. Gussenhoven W.J. and Bom N. (1991): Angle-dependent backscatter from the arterial wall. Utrasound in Med. \& Biol. 17 121-126

Kuc R. (1984): Eslimating acoustic attenuation from reflected ultrasound signals: comparison of spectral-shitt and spectral-difference approaches. IEEE Trans. Acoust. Speech and Signal Process. ASSP-32 $1-6$

Kuc R. and Schwartz M. (1979): Estimating the acoustic attenuation coetficient slope for liver from reflected ultrasound signals. IEEE Trans. Sonics and Utrason. SU-26 353-362 
Landini L. Sarnelli R. Picano E. and Salvador M. (1986): Evaluation of frequency dependence of backscatter coefficient in normal and atherosclerotic aortic walls. Uttrasound in Med. B Biol 12397. 401

Laugier P. Berger G. Fink M. and Perrin J. (1987): Diffraction correction for focused transducers in attenuation measurement in wivo. Ultrasonic Imaging 9 248-259

Linssen F.M.J. Hoeks A.P.G. and Brands P.J. (1991): The use of narrowband ultrasound for characterization of tissue in a thin layer. Phys. Med. Biol. 36 1319-1330

Lizzi F.L. Greenebaum M. Feleppa E.J. and Elbaum M. (1983): Theoretical framework for spectrum analysis in ultrasonic tissue characterization. $J$. Acoust. Soc. Am. 73 1366-1373

Lizzi F.L. Ostromogilsky M. Feleppa E.J. Rorke M.C. and Yaremko M.M. (1986): Relationship of ultrasonic spectral parameters to features of tissue microsctructure. IEEE Trans. Uitrason. Ferroelec. Freq. Contr. UFFC-33 $319-329$

Lu J.Y. and Greenleaf J.F. (1990): Ultrasonic non-diffracting transducer for medical imaging. IEEE Trans. Uitrason. Ferroelec. Freq. Contr. UFFC-37 438-447

Maklad N.F. Ophir J. and Balsava V. (1984): Attenuation of ultrasound in normal liver and diffuse liver diseases in vivo. Uttrasonic /maging $6117-125$

O'Donnel M. (1983): Effects of diffraction on measurement on the frequency-dependent ultrasonic attenuation. IEEE Trans. Biomed. Eng. BME-30 320-325

Ophir J. and Mehta D. (1988): Elimination of diffraction error in acoustic attenuation estimation via axial beam translation. Utrasonic Imaging $10139-152$

Ophir J. and Maklad N.F. (1978): A new stochastic C-scan technique for attenuation measurements in tissue equivalent material. Proc. 23rd Annu. Meet. Am. Inst. Ultrasound in Medicine. AluM Publ.

Rihyne T.L. (1977): Riadiation coupling of a disk to a plane and back or a disk to a disk: an exact solution. J. Acoust. Soc. Amer, $61318-324$

Sarnelli R. Landini L. and Squartini F. (1986): Atherosclerotic detection by ultrasounds. Appl. Path. 4 $270 \cdot 275$

Tuthill T.A. Baggs R.B. and Parker K.J. (1989): Liver glycogen and water storage: effect on ultrasound attenuation. Ulitrasound in Med. \& Biol. 15 621-627

Verhoef W.A. Cloostermans M.J.T.M. and Thijssen J.M. (1984): The impulse response of a focussed source wilh an arbitrary, axisymmetric surface velocity distribution. $J$. Acoust. Soc. Amer. 751716 . 1721

Wagner R.F. Insana M.F. and Smith S.W. (1988): Fundamental correlation lengths of coherent speckle in medical ultrasonic images. IEEE Trans. Uttrason. Ferroelec. Freq. Contr. UFFC-35 34-44 

CHAPTER 3

\section{ESTIMATION OF TRANSDUCER PARAMETERS FROM SOUND FIELD MEASUREMENTS}

The contents of this chapter is based on the publication:

Title: $\quad$ Transducer characterization from pressure amplitude distribution measurements using a Kaiman filter as parameler estimalion algorithm.

Authors: Linssen F.M.J. and Hoeks A.P.G.

In: $\quad$ Uitrasonic Imaging 12 (1990) 309-323 
Chapter 3 


\subsection{Introduction}

As already pointed out in chapter 2 , backscattered and reflected ultrasound signals depend on the sound field at the positions from where the signals are backscattered or reflected (equations 2.9 and 2.10). Much literature has appeared on the correction for the influence of the diffraction function (ODonnel 1983, Kuc and Regula 1984, Verhoef et al 1985, Laugier et al 1987). If the ultrasound signal is dominated by signals reflected at tissue interfaces, a similar correction is necessary for the influence of radiation coupling effects (chapter 2, section 2.4). The Pressure Coupling Function (PCF) depends on the sound field in the points at the surface of the reflecting surface (tissue interface). Measurement of the PCF is very cumbersome because it must be repeated for every combination of a reflector and a transducer and for every possible position of the reflector in the sound field. To investigate the influence of the position (orientation) and geometry of the reflector on the PCF, it seems more appropriate to develop a computer model from which the PCF of a given transducer/reflector combination can be calculated (chapter 4). To this end, the sound field at the reflecting surface must be calculated from a sound field model. Methods to calculate the sound field of a transducer are well known from literature (Stepanishen 1971, Penttinen and Luukkala 1976, Arditi et al 1981, Verhoef et al 1984). Some of the parameters necessary in these models, however, are unknown for a given existing transducer. In this chapter, a method will be developed by which it is possible to estimate the relevant transducer parameters from measurement of the pressure amplitude distribution in discrete points of the sound field. One of the most important factors, which influence the sound field, the Surface Velocity Amplitude Distribution (SVAD) of a transducer, in particular, is rarely known.

The SVAD may be obtained by backpropagation methods (Stepanishen and Benjamin 1982, Waag et al 1985, Schafer and Lewin 1989). Backpropagation methods are based on the relation that exists between the two dimensional spatial Fourier transforms of the pressure amplitude distributions in two parallel planes. Using this relation, the pressure amplitude distribution at the transducer surface can be calculated from the measurement of the pressure amplitude distribution in a plane parallel to the transducer surface. The relation on which the method is based, however, is only valid if the sound field is generated by monochromatic sources in continuous wave excitation (only one frequency), situated in a plane parallel to the plane where the pressure amplitude distribution is measured. Also, noise in the measurements greatly affects the results. The advantage of backpropagation methods is that the velocity amplitude is calculated at many discrete points on the transducer surface and therefore, no parametric model for the SVAD is needed. However, for some transducer geometries the SVAD may be described by a polynomial with only a few parameters. For a circular concave transducer such a model was described by Verhoef and coworkers (1984). The transducer parameters necessary for the calculation of the sound field have to be derived from measurements in a medium with parameters that are known a priori (water). Since the transducer parameters 
cannot be calculated directly from the measured data by some inverse model, it is necessary to apply a parameter estimation algorithm. A Kalman filter provides a robust method for extracting the transducer parameters from pressure amplitude measurements performed in a water tank. Its major advantage over other parameter estimation methods is the straightforward way in which the estimation process takes into account assumptions made regarding the validity of the measurements and the validity of the estimates for the parameters.

\subsection{Sound field model and measurements}

\section{2 .1 Transducer model}

The transducer used to evaluate the performance of the estimation algorithm was a circular concave transducer $(\mathrm{KB}-$ Aerotech $5 \mathrm{MHz}, 6 \mathrm{~mm}$ diameter, approximately $35 \mathrm{~mm}$ radius of curvature). The model for such a transducer, as previously described by Verhoef and coworkers (1984), assumes a circular disc, where the surface velocity $\mathrm{v}_{\mathrm{n}}\left(\mathrm{z}_{0}, \mathrm{t}\right)$ can be separated into a time-independent Surface Velocity Amplitude Distribution $f\left(z_{0}\right)$ and a position-independent velocity waveform $v(t)$ :

$$
v_{n}\left(z_{0}, t\right)=f\left(z_{0}\right) v(t)
$$

where $\mathbf{z}_{0}$ is the position of a point on the transducer surface. In this model the SVAD is assumed to be axisymmetric (Verhoef et al 1984). It may be approximated by a polynomial in $r_{0}^{2}$ :

$$
f\left(z_{0}\right)=a_{0}+a_{1} r_{o}^{2}+a_{2} r_{0}^{4}
$$

where $r_{0}$ is the radial distance from a point on the transducer surface to the axis of symmetry $\left.\left(z_{0}=\llbracket x_{0}, y_{0}, z_{0}\right]^{\mathrm{T}}, r_{0}=\sqrt{x_{0}^{2}+y_{0}^{2}}\right)$. The parameters $a_{0}, a_{1}$ and $a_{2}$ have to be estimated from measurements.

The sound pressure field at a point in the field with coordinate vector $\mathbf{z}$ can be described by a velocity potential function $\Phi(\mathbf{z}, t)$ (O'Neil 1949, Stepanishen 1981, Verhoef et al 1984, ). Under the assumptions made above, this function can be calculated by convolving the so-called diffraction impulse response function $h\left(z_{,} t\right)$ and the velocity waveform $v(t)$ :

$$
\Phi(z, t)=\iint_{S} \frac{v_{n}\left(z_{0}, t-r / c\right)}{2 \pi r} d S=v(t) * h\left(z_{t} t\right)
$$

where

$$
h(z, t)=\iint_{S} \frac{f\left(z_{0}\right) \delta(t-r / c)}{2 \pi r} d S
$$

and the integration is performed over the transducer surface $S$. 

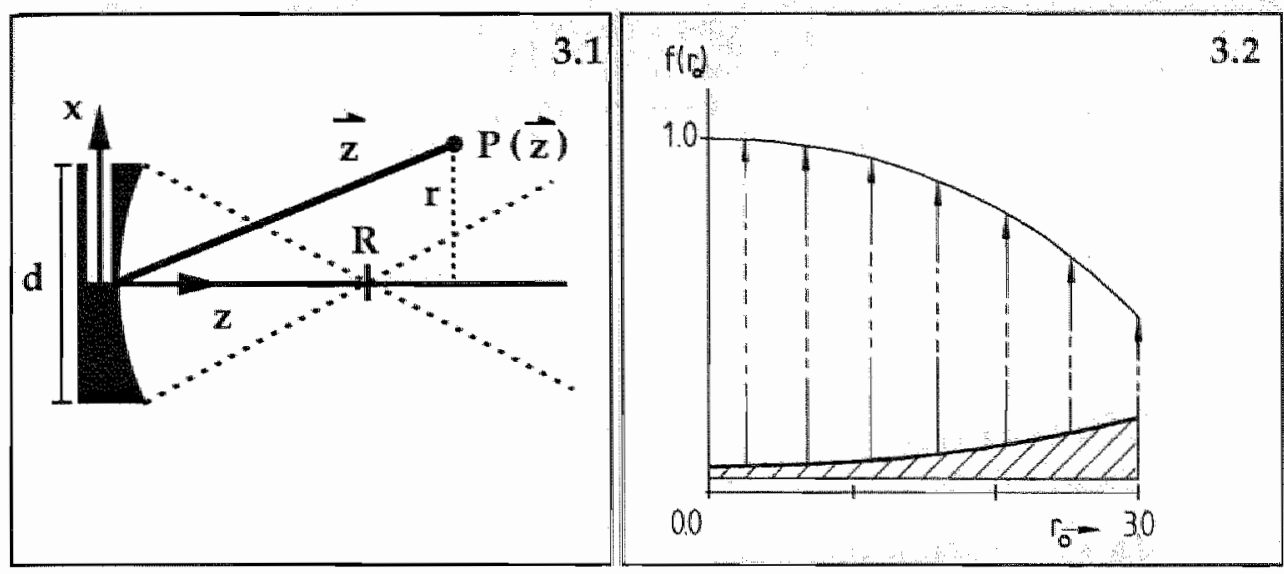

figure 3.1 Dimensions of the Iransducer.

figure 3.2 Distribution of the velocily amplitude $f\left(r_{0}\right)=1-0.044 r_{0}^{2}-0.00 * 4 r_{0}^{4}$

The impulse response $h(\mathbf{z}, t)$ depends on the geometry of the transducer (diameter and radius of curvature) and on the velocity amplitude distribution $\mathrm{f}\left(\mathrm{r}_{0}\right)$. Assuming a negligible attenuation in the medium (water), and a known velocity of sound, the transducer parameters necessary to calculate $h\left(z_{s} t\right)$ (figures 3.1 and 3.2 ) are:

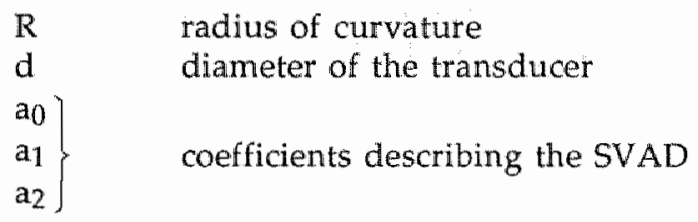

The pressure $p(\mathbf{z}, t)$ as a function of time at a certain point $z$ in the field follows from the calculated impulse response $h(z, t)$, the density of the medium $p$, and the velocity waveform $v(t)$ :

$$
p(\mathbf{z}, t)=p \frac{\partial}{\partial t}[h(z, t) * v(t)]
$$

In a continuous wave situation the velocity waveform is a harmonic signal ( $v(t)$ $=\cos (\omega t+\sigma))$. The amplitude of the expression between the square brackets in equation 3.5 can be found by calculating two values $I_{1}$ and $I_{2}$ according to the equations below:

$$
\begin{aligned}
& \mathbb{I}_{1}=\int h\left(z_{x} t\right) \cos (\omega t) d t \\
& \mathbb{I}_{2}=\int h\left(z_{x} t\right) \sin (\omega t) d t
\end{aligned}
$$


For a given frequency, differentiation with respect to time results only in a scaling of the amplitude and a 90 degree phase shift. Because only the pressure amplitude distribution is used in the remainder of this chapter, the pressure amplitude distribution $\mathbb{P}(\mathbf{z}, \omega)$ for a given frequency $\omega$ can be calculated from:

$$
P\left(z_{,}, \omega\right)=\sqrt{I_{1}^{2}+I_{2}^{2}}
$$

The impulse response function $h(z, t)$ is the result of a surface integral on the surface of the transducer (equation 3.4). This integral will be calculated using the algorithm described by Verhoef and coworkers (1984).

\subsubsection{Parameter estimation}

The Kalman filter is well known in signal analysis; it can also be used as a parameter estimation algorithm if some of the system parameters are not known (Jazwinsky 1970, Brammer and Siffling 1975). The use of the algorithm just for the determination of constants is less common, presumably because more obvious methods are available (least square, maxumum likelihood, etc.). However, these algorithms offer marginal possibilities for influencing the estimation process. A Kalman filter does offer these possibilities in a rather straightforward way, as can be understood from the explanation in this section and in section 3.2 .3 .

Considering a vector $\mathrm{x}$ containing the $\mathrm{n}$ values of the parameters, the covariance matrix $P$ of this vector and its estimate $\hat{x}\left(e . g . x^{T}=\left[R, d_{1} a_{0}, a_{1}, a_{2}\right]\right.$ ) is defined by the following expression:

$$
P=E\left((x-\hat{x})(x-\hat{x})^{T}\right)
$$

where $\mathrm{F}$ denotes the expected value operation.

The pressure amplitudes, which are measured at different positions for different emission frequencies, depend on the $n$ parameters in the vector $x$. This can be expressed as:

$$
y=g(x)+v
$$

where $\mathrm{v}$ is a vector containing $\mathrm{m}$ zero mean random values with a Gaussian distribution (noise and measurement errors). The expression $\mathbf{g}(\mathbf{x})$ is also used $\mathrm{t}$ represent the calculation of $\mathrm{m}$ pressure amplitudes gathered in the vector $\hat{y}$ which is the estimation of the measured values in $y$, using the model as described in section 3.2 (see also appendix). Conventionally, $x$ is called the state vector and its components are a function of time. Here, $x$ contains time-independent parameter values and the relation between the state vectors at the discrete times $k$ and $k+1$ is expressed as follows:

$$
x(k+1)=x(k)+w(k)
$$


In this equation, $w(k)$ is a Gaussian zero mean random vector. The covariances of the vectors $v$ and $w$ are gathered in the matrices $R$ and $Q$ :

$$
\begin{aligned}
& \mathbf{R}=E\left[v v^{T}\right] \\
& \mathbf{Q}=\mathbb{E}\left[w w^{T}\right]
\end{aligned}
$$

In the estimation algorithm the number of the iteration plays the same role as the discrete time in equation 3.9. The estimation algorithm takes the optimal estimate $\hat{x}(k-1)$ resulting from the previous iteration as a starting value $*(k)$ for the calculation of the new optimal estimate $\hat{x}(\mathrm{k})$ :

$$
\text { * }(k)=\hat{x}(k-1)
$$

and

$$
\stackrel{\mathbf{P}}{\mathbf{P}}(\mathrm{k})=\hat{\mathrm{P}}(\mathrm{k}-1)+\mathbf{Q}
$$

Here, "means a suboptimal estimate ${ }^{n}{ }^{*}$ means an optimal estimate. The filter algorithm will now try to find a vector $\hat{x}(k)$ that minimizes the trace of the matrix P from:

$$
\hat{\mathbf{x}}(k)=* * k(k)+K(k)[y-\hat{y}(k)]
$$

where $\hat{y}(k)$ is an estimate of the m measured values that can be calculated numerically from the model under the assumption that the model parameters are the $\mathrm{n}$. values gathered in the vector $x(\mathrm{k})$. The last term in equation 3.13 is called the innovation. The matrix $K(\mathrm{k})$ is solved from the equation:

$$
\left[C(k) \hat{P}^{*}(k) C(k)^{T}+R\right] K(k)^{T}=C(k) P^{*}(k)
$$

For a linear system it can be proved that the values of $\mathbb{K}(k)$, solved from the equation above, minimize the trace of the matrix $\mathbf{P}(\mathrm{k})$ (Jazwinsky 1970 , Brammer and Siffling 1975). The matrix $C(k)$ is an estimate of the derivative of the function $g(x)$, at $x=* *(k)$ (see appendix). An improved estimate of $P(k)$ can be found from:

$$
\mathrm{P}(\mathrm{k})=[\mathrm{I}-\mathrm{K}(\mathrm{k}) \mathrm{C}(\mathrm{k})] \mathbf{P}(\mathrm{k})[\mathbf{I}-\mathbf{K}(\mathrm{k}) \mathrm{C}(\mathrm{k})]^{\mathrm{T}}+\mathbf{K}(\mathrm{k}) \mathbf{R} \mathbf{K}(\mathrm{k})^{\mathrm{T}}
$$

Subsequently, the next iteration can be calculated starting at equation 3.11 .

\subsubsection{Practicall aspects}

If the starting value of $x$ is not too far from its "true" value, and if the model is not overdetermined by the parameters chosen, the algorithm will converge to a minimum variance estimate of the parameters. Overdetermination in this sense means that two or more parameters produce a similar effect on the vector $\hat{y}(k)$. In this situation, an increase in one pirameter may be compensated for by a decrease in the other parameter. 
The equations of the Kalman filter are based on the assumption that the vector $y$ depends linearly on the parameters in vector $x$. Since the model used here is not linear, it is linearized for small variations of the parameters around their actual values. The consequence is that there is an unknown interval in which parameters converge to the optimal values (minimum variance). For initial values outside this interval, the innovations resulting from equation 3.13 may be so large that some parameters do not remain in that interval. Therefore, it is useful to set boundaries between which the parameters have to be scrutinized (based on physical insight), making the problem of choosing the right initial values less critical.

The equations for the iterative part of the filter algorithm are summarized below:

Prediction:

$$
\begin{aligned}
& * x(k)=\hat{x}(k-1) \\
& \hat{P}(k)=\hat{P}(k-1)+Q
\end{aligned}
$$

Solve $\mathbf{K}(\mathrm{k})$ :

$$
\left.[C(k))^{\tilde{P}}(k) C(k)^{T}+\mathbb{R}\right] K(k)^{T}=C(k) \tilde{P}^{\tilde{P}}(k)
$$

Improve:

$$
\begin{aligned}
& \hat{x}(k)=* x^{x}(k)+K(k)[y-\hat{y}(k)] \\
& \hat{P}(k)=[I-K(k) C(k)] \tilde{P}(k)[I-K(k) C(k)]^{T}+K(k) R K(k)^{T}
\end{aligned}
$$

Considering equation $3.19 \mathrm{it}$ is obvious that the estimation process is governed by the values in the matrix $K(\mathrm{k})$. The values in this matrix greatly depend on some assumptions that have been made. The assumption made about the values in the matrix $Q$ will cause the vallues in $P(k)$ to tend to zero or to stabilize at a certain level (equation 3.17). If the values in $\mathrm{CPC}^{\mathrm{T}}$ are small compared to those of the matrix $R$ it can be seen from equation 3.18 that the values of $K(k)$ are proportional to those of $P(k)$. Because the Kalman filter will try to minimize the values of $\mathbf{P}(\mathrm{k})$, the innovation process will abort itself because the values in $\mathbf{K}(\mathrm{k})$ will decrease too (equation 3.18). To avoid abortion it is necessary to set the diagonal values in $\mathbf{Q}$ to a minimum value. These minimum values are the squares of the errors allowed in the values of the corresponding parameters in $\mathbf{x}$. Then the right hand side of equation 3.18 will always contain non-zero values because of equation 3.17 .

The estimation process also depends on the assumption made regarding the measurement noise. Assuming very accurate measurements and thus setting the values in the matrix $\mathbb{R}$ at very low values, $K(k)$ will be almost equal to $\mathbf{C}(k)^{-T}$. This will result in drastic correction the parameter estimates. Of course, this is 


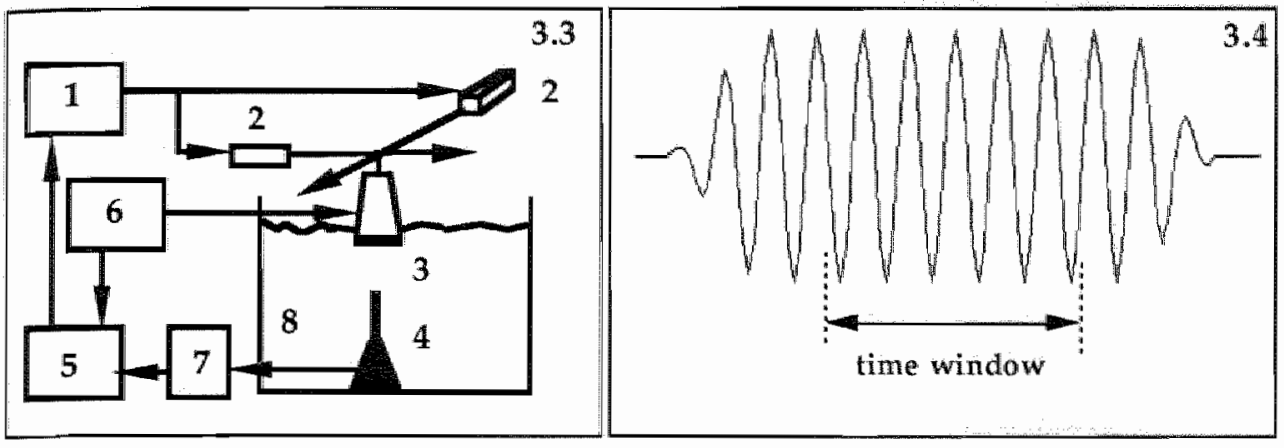

figure 3.3 Measurement set-up. $1=$ Torch minicomputer, $2=$ stepper motors, $3=$ transducer, $4=$ PVDF hydrophone, $5=$ peak detector, $6=$ signal generator, $7=$ preamplifier, $8=$ water tank.

figure 3.4 Typical example of a received hydrophone signal. The delay time and length of the window can be chosen to ensure measurement of the pressure amplifude in the part of the signal reflecting the continuous wave situation.

very dangerous if the assumption about accuracy is wrong or if the system is strongly nonlinear. In general, the best policy is to use $\mathbf{R}$ for what it originally represents: an estimate of the deviations in the measurement.

\subsubsection{Measurements}

The "continuous wave" pressure amplitude distribution in a plane perpendicular to the ultrasound beam axis is measured using a PVDF hydrophone with a small aperture $(0.6 \mathrm{~mm})$. The transmitting probe is mounted on an $\mathrm{x}-\mathrm{y}$ table construction with two computer controlled stepper motors (figure 3.3). The size of the steps is $0.1 \mathrm{~mm}$ in both directions. A peak detector measures the peak amplitude of the received hydrophone signal. The emission frequency is selected manually. By setting the appropriate delay and window length of the peak detector it is possible to select a part of the hydrophone signal that represents the continuous wave situation (figure 3.4). During this time window the transient effects of the frequency response of the transducer are negligible. The pressure amplitudes, measured in $\mathrm{dB}$ 's, with discrete steps of $0.375 \mathrm{~dB}$, are stored by the same computer used to control the stepper motors.

After the hydrophone has been positioned by hand at a certain distance from the transducer, the computer searches for the center of the beam (i.e. the position with the highest pressure amplitude). This position becomes the center of a square plane that is scanned $(6.4$ by $6.4 \mathrm{~mm}$ in steps of $0.1 \mathrm{~mm})$. The amplitude values measured at each position are averaged ( 4 times) and stored for further processing. To obtain a three-dimensional pressure amplitude distribution for several frequencies within the bandwidth of the transducer the pressure amplitude distribution was scanned in planes at distances of $21,27,33,39$ and $45 \mathrm{~mm}$ 


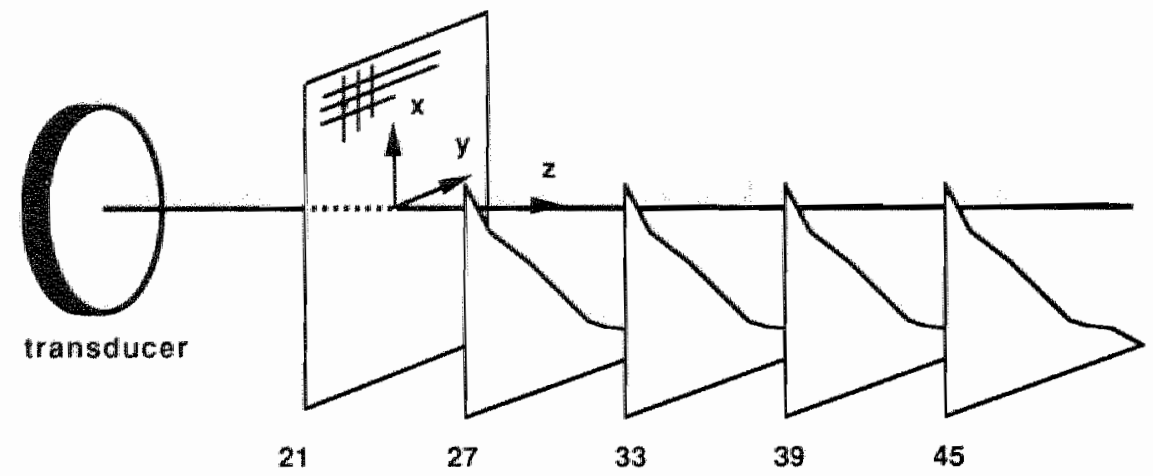

figure 3.5 Contiguration of the five planes scanned at discrete points situated on a $0.1 \mathrm{~mm}$ grid in both directions. The planes were scanned at emission frequencies of $3.5,5.0$ and 6.5 $\mathrm{MHz}$.

from the transducer (figure 3.5). The emission frequencies were $3.5,5.0$ and 6.5 $\mathrm{MHz}$. The velocity of sound was found to be approximately $1484 \mathrm{~ms}^{-1}$.

The computer model assumes a sound field with a circular symmetry. Indeed, there were no significant deviations visible in the pressure amplitude distributions (beam plots) of the scanned planes. In order to obtain a vast data reduction as well as some smoothing of the data, the following steps are taken: first, the "real" position of the center of the beam in a scanned plane is determined by averaging the $x$ and $y$ coordinates of the points where the measured level is less than $6 \mathrm{~dB}$ below the maximum level in that plane. Subsequently, the distances between the center of the beam and the points where a given level has been measured are averaged. In this way, the radial distances belonging to 12 amplitude levels in the main lobe are determined for each of the scanned planes and emission frequencies. The measurement vector $\mathrm{y}$ is structured as shown below:

$$
\begin{aligned}
& y=l y(1,1,1) \ldots y(1,1,12), \quad \quad \ldots . . \quad \text {,y }(1, n p, 1) \ldots y(1, n p, 12), \\
& \text { plane } 1 \text { plane np } \\
& \text { frequency } 1 \\
& y(n f, 1,1), \quad \ldots \quad \text {,y }(n f, n p, 1), \ldots, y(n f, n p, 12)] \\
& \text { plane } 1 \text { plane np }
\end{aligned}
$$

\section{frequency $\mathrm{nf}$}

where $y(i, j, 1)$ is the pressure amplitude, measured in the $j$-th plane, for the $\mathrm{i}$-th emission frequency, at the 1 -th radial distance. 
The model can calculate the pressure amplitudes in $y$ at the radial and axial distances that are determined as described above. This calculation is performed as described in section 3.2.1. Because the peak detector results are available in dBs, the computed pressure distribution resulting from equation 3.6 is also transformed to a $\mathrm{dB}$ scale.

\subsection{Results}

The algorithm and the transducer model were implemented on the same computer that was used for the measurements. A first run of the algorithm on the data acquired showed that the model was overdetermined if the diameter of the transducer $d$ as well as the values $a_{1}$ and $a_{2}$ were included in the state vector $x$. Because the diameter is easily measured, this parameter was excluded from the estimation process. Scaling of the velocity amplitude would only result in a proportional scaling of all the pressure values. Therefore, ao was excluded from the estimation process too. The value of an was set to one. The vector $x$ therefore contained the parameter $a_{1}, a_{2}$ and $R$.

A typical example of an estimation process is shown in figure 3.6. It can be seen. that the estimation process will not result in a single stable estimate, but that the values vary around some ultimate value. This is caused by the fact that the values of the matrix $\mathbf{Q}$ were set at a minimal value, in order to keep the innovation process going (see section 3.2.3). Also, it can be seen that there is no significant innovation of the estimates after the tenth iteration. In this example, there was simply an upper limit to the number of iterations, but it is possible to define an abortion criterion based on the values in $P(k)$ or the relative change in the estimate. Figure 3.7 shows the difference between the calculated values (based on the last estimate) and the measured values. Taking into account that the discretization level is $0.375 \mathrm{db}$ (dashed line), the results are very good.

A more illustrative figure is figure 3.8 , where the calculated beam profiles are compared to those measured. The main difference between the calculated and the measured beam profiles is that the amplitudes measured outside the main lobe are systematically higher. This is caused by the fact that a peak detector was used for the amplitude measurement. Any additional noise will give rise to a positive bias in the measurement, particularly if the signal-to-noise ratio is low. The positions of the side lobes agree very well. In figure 3.8 , a horizontal bar is drawn representing the width of the aperture of the hydrophone. It is obvious that the aperture is very wide in comparison with the beamwidth. This is a source of systematic measurement errors at positions where the pressure distribution changes rapidly within the aperture (e.g. at the local minima).

\subsection{Discussion}

It can be concluded that the Kalman filter is an adequate parameter estimator for a system in which the influence of the parameters is not easy to predict. The facthat the resulting estimate varies around some mean value may be regarded as a disadvantage, but it also provides insight into the validity of the model. It 

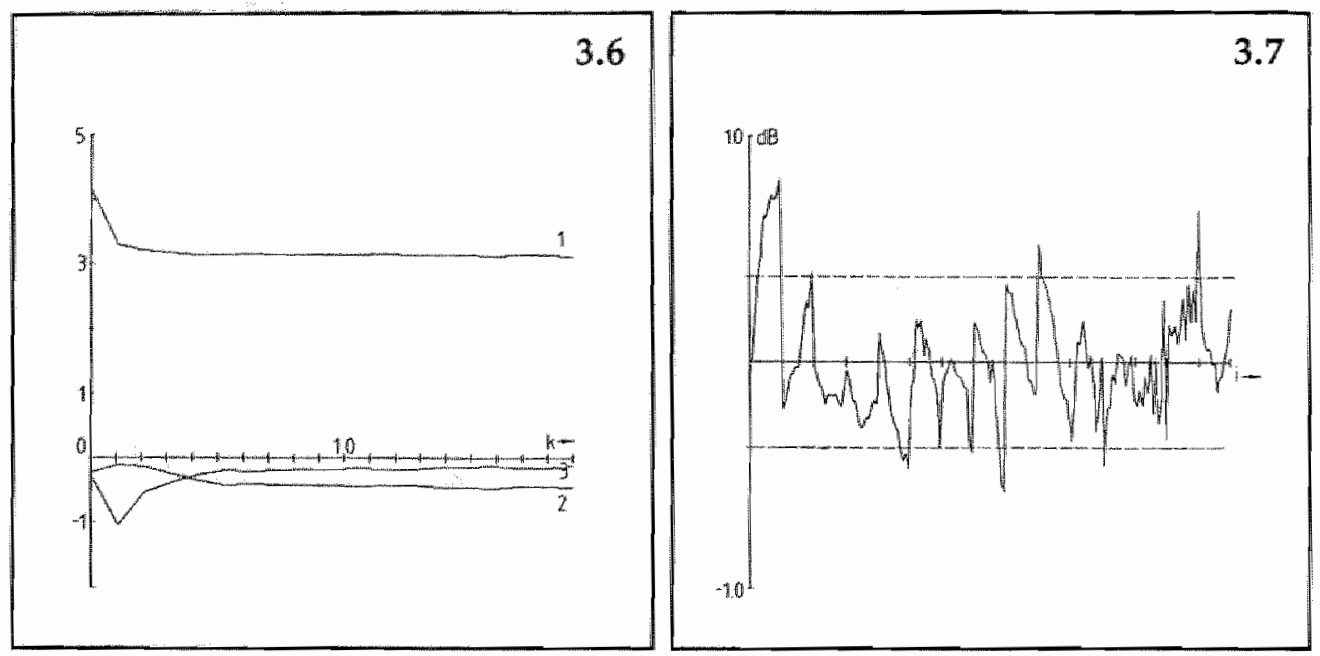

figure 3.6 The estimation process. The values of the estimates after the $k$-th iteration are plotted as curves. The parameters plotted are $\mathrm{A}_{\mathrm{x}} \mathrm{a}_{1}$ and $\mathrm{a}_{2}$ (lines 1,2 and 3 respectively).

figure 3.7 Difference between 180 measured values $y(i)$ and the corresponding calculated pressure amplitudes $\hat{y}(i)$ in $\mathrm{dB}$ 's.
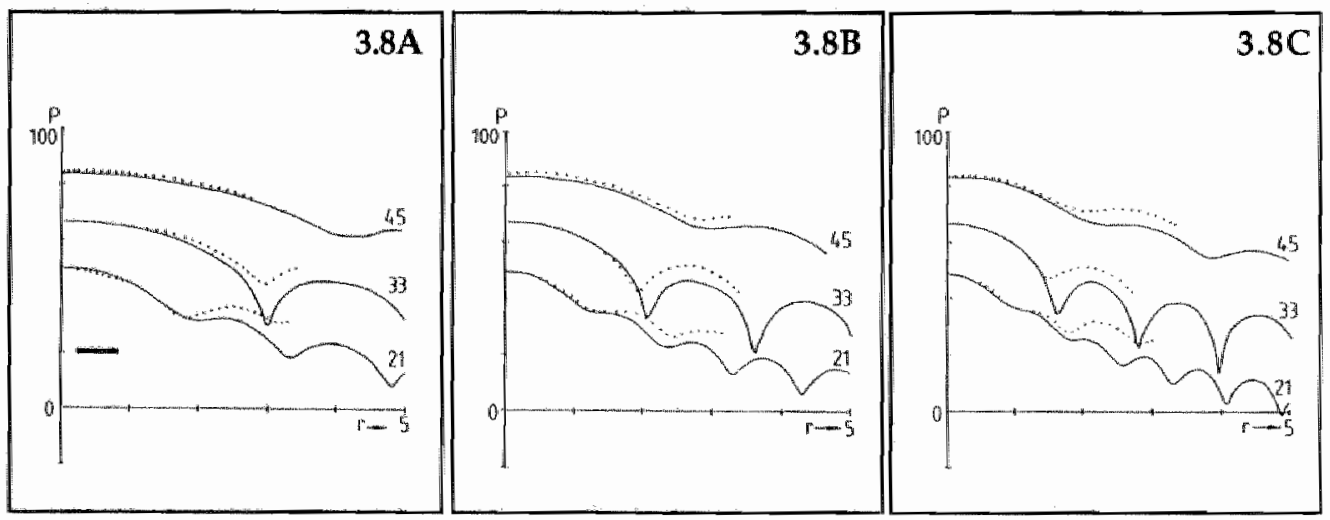

figure $3.8 \quad$ Measured (...) and calculated $(\ldots)$ beam profiles. The distance to the transducer is given in the ligure. The three ligures are at emission frequencies of $3.5,5$ and 6.5 $\mathrm{MHz}$, respectively. 
shows the significance of the different parameters for the calculation of the sound field. An important parameter does not exhibit great variations as this will produce great deviations between the measurement and the outcome of the model.

Unlike the backpropagation method, this method estimates a Surface Velocity Amplitude Distribution (SVAD) based on measurements over the whole region of interest, whereas the backpropagation method calculates the SVAD based on measurement in a single plane. Also, the backpropagation method does not combine the measurements obtained at different frequencies. The backpropagation method calculates a different SVAD for every emission frequency. The results of the method proposed in this paper suggest, however, that the SVAD does not depend greatly on the emission frequency. The results presented in this paper validate transducer models based on the calculation of the impulse response assuming a SVAD independent of the emitted waveform.

Another great advantage of the method proposed is its relatively low sensitivity to random (zero mean) errors in the measurement. In particular, the mechanical accuracy of the measurement set-up can be much lower than is necessary for the backpropagation method, which is very sensitive to measurement noise and undersampling (Schafer and Lewin 1989).

In the method presented, two sources of systematic measurement errors can be identified: the width of the hydrophone aperture with respect to the local inhomogeneity of the field, and the method of amplitude measurement. It should be possible to correct for the effect of the width of the hydrophone by averaging the calculated pressure amplitudes over the hydrophone surface instead of calculating the pressure amplitude at the center of the hydrophone. This was not done because it would not result in a major improvement of the estimated parameter values, because these are estimated from pressure amplitude values in the main lobe. The effect of the use of a peak detector for the amplitude measurement cannot be taken into account in the model, but it should be possible to use a different method of amplitude measurement. However, in view of the fact that the main lobe can be calculated very accurately in the region examined, this seems superfluous.

The overall agreement between measured and calculated data (especially for the main lobe) suggests that it should be possible to calculate the sound field at a reflecting surface for different frequencies. From this it may be possible to calculate the effect of the position and geometry of a reflector on the frequency content of a reflected ultrasound pulse (Pressure Coupling Function, see chapter 4). The transducer parameters found may be used for the calculation of the near field, but this is a very tentative operation. Calculation of the sound field is most reliable in the region actually scanned. The medium parameters necessary for this computation (velocity of sound, attenuation, density) can be found in the literature, or, if possible, may be obtained by measurement. A far more serious problem is encountered when the reflecting properties of tissue interfaces have to be modelled. Since not much literature is available on this subject, the problem needs further investigation. 
The results are a validation of the transducer model proposed by Verhoef and coworkers (1984) for circular concave transducers. The assumption that the SVAD does not depend on the emission frequency of emitted waveform seems to be valid. The method of measurement and estimation proposed in this chapter yields reliable results allowing calculation of diffraction and radiation coupling effects (see chapter 4 ). The parameter estimation algorithm is very robust and the requirements regarding the accuracy of the scanning equipment are not as great in comparison with other methods.

\section{References}

Arditi M. Foster F.S. and Hunt J.W. (1981): Transient fields of concave annular arrays. Utrasonic Imaging $377-61$

Brammer $K$. and Siffling G. (1975): Kalman Bucy Filter: deterministische Beobachtung und stochastische Filterung. ( $\mathrm{A}$. Oldenbourg, Munchen and Wien 1975) (in German).

Jazwinsky A.H. (1970): Stochastic Processes and Filtering Theory. (Academic Press, New York 1970).

Kuc $R$ and Regula ir. D.P. (1984): Diffraction effects in reflected ultrasound spectral estimates. IEEE Trans. Biomed. Engin. BME-31 537-545

Laugier P. Berger G. Fink M. and Perrin J. (1987): Diffraction correction for focused transducers in attenuation measurement in vivo. Uitrasonic Imaging 9 248-259

Linssen F.M.J. and Hoeks A.P.G. (1990): Transducer characterization irom pressure amplitude distribution measurements using a Kalman filter as parameter estimation algorithm. U/trasonic Imaging 12 $309-323$

O'Donnel M. (1983): Effects of diffaction on measurement on the frequency-dependent ultrasonic attenuation. IEEE Trans. Biomed. Engin. BME-30 320-325

O'Neil H.T. (1949): Neartield and tarfield of pulsed acoustic radiators. J. AC. Sac. Am. 21 516-526

Penttinen $A$ and Luukkala $M .(1976)$ : The impulse response and pressure neartield of a curved ultrasonic radiator. U. Phys. D. 9 1547 -1557

Schafer M.E. and Lewin P.A. (1989): Transducer characterization using the angular spectrum method. d. Acoust. Soc. Amer. 85 2202-2214

Stepanishen P.R. (1971): Transient radiation from piston in an infinite ptanar baffle. J. Acoust. Soc. Amer. 49 270-275

Steparishen P.F. (1981): Acoustic transients from planar axisymmetric vibrators using the impulse response approach. J. Acoust. Soc. Amer. 70 1176-1181

Slepanishen P.R. and Benjamin K.C. (1982): Forward and backward projection of acoustic fields using FFT methods. J. Acoust. Soc. Amer. 71 803-812

Verhoer W.A. Cloostermans M.J.T.M. and Thijssen J.M. (1985): Diffraction and dispersion effects on the estimation of ultrasound attenuation and velocity in biological tissues. IEEE Trans. Biomed. Engin. BME-32 521.529

Verhoef W.A. Cloostermans M.J.T.M. and Thijssen J.M. (1984): The impulse response of a focused source with an arbilrary axisymmetric surface velocity distribution. J. Acoust. Soc. Amer. 751716 1721 
Waag R.C. Campbell J.A. Fidder J. and Mesdag P.P. (1985): Cross-Sectorial measurements and ex. trapolations of ultrasonic fields. IEEE Trans. Sonics Uitrasonics SU-32 26-35 


\section{Appendix}

The Kalman filter needs a model by which it is possible to predict the measured data, given an estimate for the unknown parameters. Here, the model used to calculate the diffraction impulse response function $h(\mathbf{z}, t)$ in a point $\mathbf{z}$ is the one described by Verhoef et al (1984). The pressure amplitude at the different emission frequencies is calculated according to the derivations in section 3.2. At the point $z$ the pressure amplitude of the sound field is measured with a hydrophone. The formal expression which represents the model to relate the set of unknown parameters $x$ and the actual pressure amplitude values $y$ is expressed as:

$$
y=g(x)
$$

where $\mathrm{g}$ is a functional expression.

Further, to improve the estimate of the parameters, the Kalman filter needs the sensitivity (matrix C) of the functional $\mathrm{g}$ with respect to the parameter values at $x=\hat{x}:$

$$
C=\frac{\partial g}{\partial x} \quad \text { at } x=\hat{x}
$$

If $\mathbf{g}(\mathbf{x})$ is an analytical expression, it is possible to calculate the derivative of $\mathbf{g}(\mathbf{x})$ with $x=\hat{x}$. Here, we use a numerical model to calculate the pressure amplitude values. A first order estimate of the matrix $\mathrm{C}$ is obtained by perturbation of the different parameter values. The $\mathrm{i}$-th column of $\mathbf{C}, \mathbf{C}_{\mathbf{i}}$, is then:

$$
C_{i}=\frac{g\left(\hat{x}_{i}\right)-g(\hat{x})}{\Delta x_{i}}
$$

where $\hat{\mathbf{x}}_{i}$ is equal to $\hat{\mathbf{x}}$, except that the $\mathrm{i}$-th parameter is augmented by $\Delta \mathrm{x}_{\mathrm{i}}$. 
CHAPTER 4

\section{COMPUTATION OF PRESSURE COUPLING FUNCTION}

This chapter is based on a manuscript, which has been accepted for publication:

Title: Computation of the signal distortion of reflections from specular reflectors by impulse Auy modeling.

Authors: Linssen F.M.J. and Hoeks A.P.G.

In: Journal of the Acoustical Society of America 
Chapler 4 


\subsection{Introduction}

In order to determine the acoustical properties of a tissue from signals obtained from specular reflectors, it is necessary to be aware of the influence of the Pressure Coupling Function (PCF) on the data. The amplitude spectrum of a broad band pulse may be distorted by a non-uniform Pressure Coupling Function and the amplitude spectrum of a sufficiently narrowband signal may be amplitude modulated (see chapter 2). Investigation of the characteristics of the PCF from experiments is a time consuming task, because measurements must be repeated for every reflector at every possible position in the sound field. Therefore, a computer simulation model based on a sound field model, using realistic transducer parameters is more appropriate. In this chapter a method will be presented that allows computation of the PCF if the transducer and reflector configurations are known. Apart from the geometry of the reflector and the dimensions of the transducer, the PCF depends on the sound field at the transducer surface (the reflection coefficient is not taken into account in the PCF, see equation 2.14). To obtain realistic simulations, the parameters of a common medical ultrasound transducer are used for the calculation of the sound field. A method to estimate these transducer parameters for an existing transducer was described in chapter 3. There, for example, it was found that the Surface Velocity Amplitude Distribution, (SVAD) is non-uniform, in contrast to what is generally assumed. In practical situations the unknown influence of the tissue and lack of information about the geometry of the reflecting tissue interface do not allow an exact calculation, but it may be possible to perform some first order corrections on the data or, alternatively, find a measurement situation in which the influence of the PCF is minimal.

The theoretical background for the calculation of the sound field is well known (Stepanishen 1971, Penttinen and Lukkala 1976, Verhoef et al 1984, Sung 1989, Ocheltree and Frizzell 1989) and for some special transducer/reflector configurations it is possible to arrive at an analytical solution for the Radiation Coupling Function (RCF, Rhyne 1977). The RCF is the integral of the impulse response function over a surface. In order to be independent of the restrictions on geometry and some other assumptions necessary to calculate an analytical solution, a numerical procedure for solving the problem will be presented. The procedure used is a modification of the impulse-ray method as described by Cassereau and Guyomar (1988). The major difference between the two methods is in the distribution of the acoustical energy in space. The method proposed in this chapter is straightforward and allows a great reduction in computation time. To test the procedure, the RCF of a configuration, for which an analytical result was available, was calculated. The results derived from the numerical approach were in agreement with the theoretical results. The influence of some parameters on the accuracy of the calculated RCF will be discussed. From the RCF it is possible to calculate the Pressure Coupling Function (PCF) by performing a differentiation with respect to time. The PCF can be used to calculate the dis. tortion of a given waveform after reflection. This distortion will be investigated 
in the frequency domain. The PCF's of some transducer/reflector combinations will be calculated and the results discussed.

\subsection{Derivation of numerical model}

\subsubsection{Radiation and pressure coupling}

The sound field is determined by the velocity potential function. The velocity potential 0 in a point $z$ in front of a transducer with surface $S$ is calculated from the Ralyleigh surface integral over $S$ (Verhoef et al 1984):

$$
\Phi(z, t)=\iint_{S} \frac{v_{n}\left(z_{0}, t-r / d\right)}{2 \pi r} d S
$$

Here, $v_{\mathbf{n}}\left(\mathbf{z}_{0}, \mathbf{t}\right)$ is the normal surface velocity at a point of the transducer and $\mathrm{r}$ is the distance between the point with coordinates $\mathbf{z}$ and a point on the transducer surface $z_{O}$. The velocity of sound is $c$ and the attenuation is assumed to be zero.

If the normal velocity $w_{n}$ can be expressed as the product of an amplitude distribution $f\left(z_{0}\right)$ and a velocity waveform $v(t)$, the expression for $\Phi\left(z_{,}, t\right)$ becomes:

$$
\Phi(z, t)=v(t) * \iint_{S} \frac{f\left(z_{0}\right) \delta(t-r / c)}{2 \pi r} d S=v(t) * h(z, t)
$$

Here, $r$ is the distance between $z$ and $z_{0}$ and * denotes the convolution operation. The surface integral over the transducer surface is a function, $h(z, t)$, of the position $z$ in the field and of time $t$ (chapter 3 ). This function is called the diffraction impulse response at point $z$. The separation of the normal velocity $v_{n}$ into a Surface Velocity Amplitude Distribution (SVAD) and a velocity waveform $v(t)$ was validated by the results of chapter 3 : the calculated pressure amplitude distribution of the sound field using only one SVAD was consistent with the measured pressure amplitude distribution for the whole frequency bandwidth of the transducer. The pressure function at a point $\boldsymbol{z}$ as a function of lime is derived from the velocity potential at that point according to:

$$
p(\mathbf{z}, t)=\rho \frac{\partial \Phi(\mathbf{z}, \mathbf{l})}{\partial t}
$$

where $p$ is the density of the medium.

For $v(t)$ being a Dirac function, the waveform as detected by a pressure sensitive surface $A$ in the sound field can be calculated from a surface integral operation. 
over that surface. Taking into account the local sensitivity in reception $f_{r}(z)$, it is possible to calculate the Pressure Coupling Function PCF(t):

$$
\operatorname{PCF}(t)=\iint_{A} f_{r}(z) p(z, t) d A
$$

Using equations 4.2 and 4.3 , the PCF is calculated by differentiating the Radiation Coupling Function $\mathrm{RCF}(\mathrm{t})$ :

$$
P C F(t)=\rho \frac{\partial R C F(t)}{\partial t}
$$

where the Radiation Coupling Function is calculated from:

$$
\operatorname{RCF}(t)=\iint_{A} f_{r}(z) h(z, t) d A
$$

The deformation of the signal waveform $v(t)$ can be regarded as a linear filtering process operating on that waveform (see equation 2.10). The amplitude spectrum of the reflected waveform is calculated from the amplitude spectrum of the emitted waveform by multiplication with the amplitude spectrum of the Pressure Coupling Function. The differentiation with respect to time (equation 4.5 ) is equivalent to a multiplication by jo in the frequency domain. Therefore, in the frequency domain, the filter can be calculated from the spectrum of the RCF according to:

$$
\operatorname{PCF}(f)=j \omega F|\operatorname{RCF}(\mathrm{t})|
$$

where $F$ denotes the Fourier transform operation and $\omega=2 \pi f$.

\subsubsection{Calculation of the Radiation Coupling Function}

A reflector in front of a transducer returns part of the emitted acoustical waves to the transducer surface, resulting in a response in a part of the transducer surface. This process can be described by a radiation coupling process, but now the geometry and the reflecting properties of the reflector have to be taken into account. The pressure sensitive surface in equation 4.4 and 4.6 is then the transducer. Rhyne (1977) has calculated the Radiation Coupling Function (RCF) for a configuration of a flat circular transducer and a flat parallel plane in front of it (figure 4.1). If the transducer and the reflector have an arbitrary geometry, it is hardly possible to find an analytical expression for the RCF. Therefore, Cassereau and Guyomar (1988) proposed a numerical approach which they called the impulse ray model. A similar approach will be presented here. 

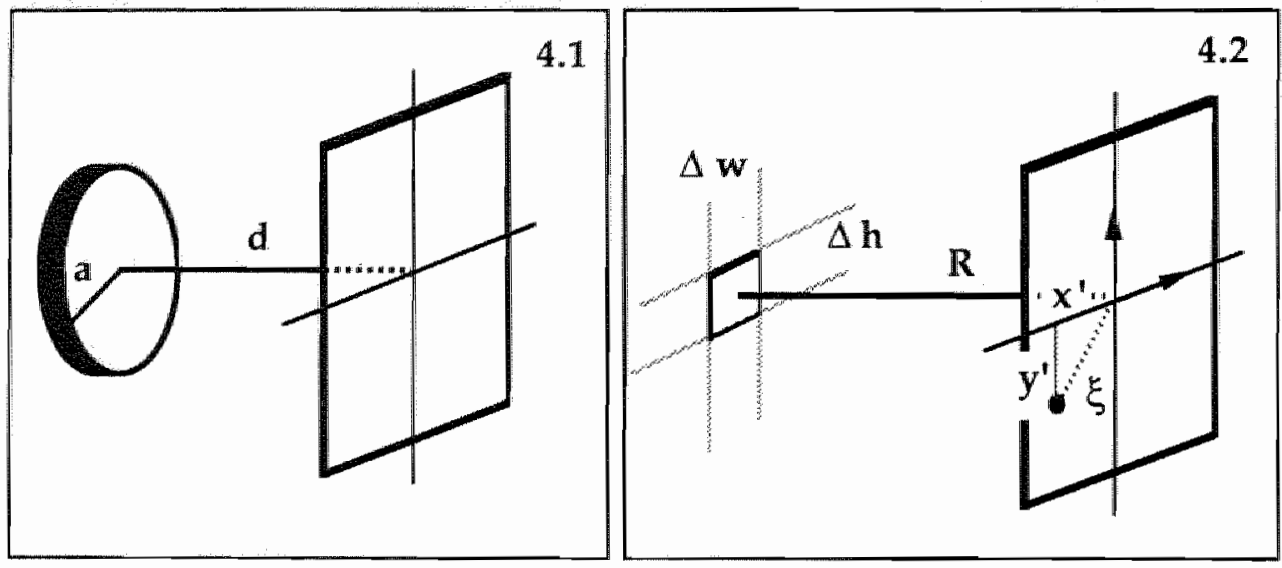

figure 4.1

Planar reflector in tront of a flat circular transducer, a = radius of transducer, $d=$ distance between reflector and transducer

figure 4.2 Elementary source, dimension $\Delta \mathrm{h} * \Delta \mathrm{w}$. Point at axial distance $\mathrm{R}$ and llateral distance $\xi$

The surface integral in equation 4.2 , from which the impulse response at a particular point is calculated, will be approximated by dividing the transducer surface into small elementary sources and summing the impulse response functions from all elementary sources (Sung 1989). Under certain conditions (see section 4.2.4) it is allowed to regard these sources as point sources. The radiation of acoustical waves is then modeled by a number of acoustical rays originating from the center of each elementary source. Each ray has a certain strength and orientation. The reflection of the acoustical rays at a reflecting surface is modeled using simple reflection laws. The path that a ray follows after reflection can be easily calculated. If a ray relurns to the transducer surface the contribution of that ray to the RCF will be calculated.

Cassereau and Guyomar attributed a number of rays to each elementary source and calculated the path of every ray after reflection at the reflecting surface. The orientation of the rays was fixed and the same for every elementary source. Then for every ray the point where it intercepts the reflecting surface must be calculated. In the approach presented in this chapter, the reflector is also divided into small elementary reflectors. It is then possible to calculate the path of all the rays which originate from the center of an elementary source and travel to the center of every elementary reflector. Hence, the orientation of the rays is different for every elementary source, and therefore every ray has a weighing factor according to its strength. This process is then repeated for every elementary source. The contribution dRCF $(k, 1, t(k, l))$ to the RCF of the $k$-th elementary source in combimation with the 1-th elementary reflector depends on the strength of the elementary source, the portion of the acoustical energy that arrives at the l-th elementary reflector and the sensitivity of the transducer at the point where the reflected 
ray arrives. Summation of the contributions of all source/reflector combinations will yield the RCF as a function of time:

$$
\operatorname{RCF}(\mathrm{t})=\sum_{\mathrm{k}} \sum_{1} \mathrm{dRCF}(\mathrm{k}, \mathrm{l}, \mathrm{t}(\mathrm{k}, \mathrm{l}))
$$

Here, $t(k, 1)$ is the time, counting from the emission of the pulse, when the ray traveling from $k$ to $l$ arrives back at the transducer surface.

\subsubsection{Calculation of dRCF}

The strength $\phi(k)$ of an elementary source is proportional to the local value $f_{s}(k)$ of the surface velocity amplitude distribution (SVAD) and the area $\mathrm{dA}_{\mathrm{S}}(\mathrm{k})$ of this elementary source:

$$
\phi(k)=f_{S}(k) d A_{S}(k)
$$

For a non-uniform SVAD, $\mathrm{f}_{\mathrm{S}}(\mathrm{k})$ is taken in the center of the elementary source.

A point source distributes its acoustical energy uniformly in all directions. Therefore, the amplitude $A$ of a spherical wave is inversely proportional to the distance traveled:

$$
A=A_{0} \frac{r_{0}}{r}
$$

where $A_{0}$ is the amplitude at distance $r_{0}$ from the source.

If the elementary reflector is regarded as a source, the strength $\gamma(\mathrm{k}, \mathrm{l})$ of this source is proportional to its area $\mathrm{dA}_{\mathrm{r}}(1)$ and the normal component of the velocity amplitude of the impinging waves (Hottel and Sarofim 1967). With $\alpha$ the angle between the vector pointing from the center of the elementary source to the center of the elementary reflector and the direction of the normal vector $\mathbf{n}$ on the surface, the expression for the source strength becomes:

$$
\gamma(k, 1)=\frac{f(k) d A_{r}(1) \cos (\alpha)}{2 \pi r}
$$

The parameter description of a reflected ray originally traveling from a point with coordinate vector $\mathbf{z}_{1}$ on the transducer to a point with coordinate vector $\mathbf{z}_{2}$ on the reflector is expressed as:

$$
\mathbf{z}=z_{2}+\lambda\left(\left(z_{2}-z_{1}\right)-2\left[\left(z_{2}-z_{1}\right) \cdot n\right] \mathbf{n}\right)
$$

where $\lambda$ is the parameter and $\mathbf{n}$ is the normal vector with unit length. 
It is then possible to calculate the point $z_{\mathbb{r}}$ where the reflected ray hits the transducer surface.

The contribution dRCF( $k, 1, t(k, l))$ to the $R C F$ of the $k$-th elementary source in combination with the $1-$ thi reflector $\operatorname{dRCF}\left(k_{p} l\right)$ is calculated from:

$$
\operatorname{dRCF}\left(k_{,}, 1,4(k, l)\right)=\gamma(k, l) f_{r}\left(z_{1}\right)
$$

where $f_{I}$ is the pressure sensitivity at the point $z_{r}$, where the ray from point $k$ to point 1 hits the transducer. Here it is assumed that for sufficiently small elementary reflectors, the incoming wavefront can be regarded as a plane wave. The wave, reflected by the elementary reflector and its neighbours is then also a plane wave. The contributions of the distinct elementary reflectors to the reflected wave are them regarded as pencil-shaped beams, of which the amplitude does not decrease with distance.

The requirement that the space angle over which the rays are reflected is limited (pencil shape beam) results in a constraint on the dimension $\Delta \mathrm{d}$ of the elementary reflectors. This constraint is derived in the appendix. The result is:

$$
\Delta \mathrm{d} \leq \frac{\kappa-\gamma}{1+\gamma} \cdot \Delta \mathrm{w}
$$

where $k \Delta w$ is the maximum dimension of the area where the rays arrive (see appendix).

\subsubsection{Constraints}

An aspect that has to be taken into account is the validity of the assumption that the subareas on the transducer can be regarded as point sources. Ocheltree and Frizzel (1989) showed that the deviation from the point source assumption for a reclangular elementary source (hamonic excitation) can be expressed by a factor Q:

$$
Q=\operatorname{sinc}\left(\frac{k x^{\prime} \Delta h}{2 R}\right) \operatorname{sinc}\left(\frac{k y^{\prime} \Delta h}{2 R}\right)
$$

where $\mathbb{R}$ is the distance (along the $z$-coordinate) from the point in the field to the center of the subarea. $\Delta h$ and $\Delta w$ are the dimensions of the subarea in the $x$ and $y$ directions respectively, and $k$ is the wavenumber $k=2 \pi f / c$, where $f$ is the excitation frequency). $x^{\prime}$ and $y^{\prime}$ are the lateral distances from the point in the field to the center of the subarea in the $x$ and $y$ directions, respectively (figure 4.2). If $\Delta \mathrm{h}$ and $\Delta w^{\prime}$ are of equal size, and under the assumption that $x^{\prime \prime}$ and $y^{\prime}$ are of the same order of magnitude, the maximum value of the argument of the sinc functions has to be 0.24 if one wishes to keep the deviation below 2 percent. The maximum value of $\mathrm{k}$ follows from the maximum frequency within the bandwidth of the transducer. The minimum value for $\mathbb{R}$ follows from the minimum distance 
from a point on the transducer surface to the reflector. The maximum value of $\Delta \mathrm{w}$ and $\Delta \mathrm{h}$ then follow from:

$$
\Delta W=\Delta h \leq 0.24 \frac{2 \mathrm{R}}{\mathrm{x}_{1} \mathrm{k}}
$$

where $\mathrm{x}_{1}$ is the estimated maximum lateral distance between a point on the transducer and a point on the reflector.

A similar constraint on the size of the elementary sources is found in the same paper by Ocheltree and Frizzel, which states that the reflector should lie in the far field of the elementary source. With $\lambda$ the shortest wavelength to be expected, an expression for $\Delta \mathrm{w}$ and $\Delta \mathrm{h}$ is found from:

$$
\Delta w=\Delta h \leq \sqrt{\frac{4 \lambda R}{F}}
$$

where $\mathrm{F}$ is an arbitrary factor (typically larger than 10 ).

\subsection{Algorithm}

\subsubsection{Basic description}

For a transducer and a reflector with a simple geometry it is easy to create a regular distribution of sample points. The coordinates of the centers of the transducer subareas and the values of the areas are stored in arrays. In addition to the coordinates of the center of each reflector subarea, the value of the area and the normal vector are calculated.

A straightforward implementation of equation 4.11 will take a large amount of computation time (proportional to the number of reflector elements times the number of source elements). However, there are many combinations of elementary sources and elementary reflectors that do not contribute to the RCF because the reflected ray does not return to the transducer surface. If the transducer is a smooth surface with a concave edge, it can be seen that those subareas on the reflector for which the line 1 does not intercept the transducer surface will not contribute to the RCF. Line $l$ is a line through the center of the subarea in the direction of the normal vector in the center of the reflector (see figure 4.3). On the basis of this criterion it is possible to select only the relevant points on the reflector.

Also, for a given elementary reflector the points contributing to the RCF on a smooth transducer surface are situated in a single closed region. One point that definitely lies within this region is the point where line 1 , described as above, intercepts the transducer surface. If the transducer points are on a regular grid it is possible to scan this region, starting at the discrete point on the surface nearest to the interception point. 

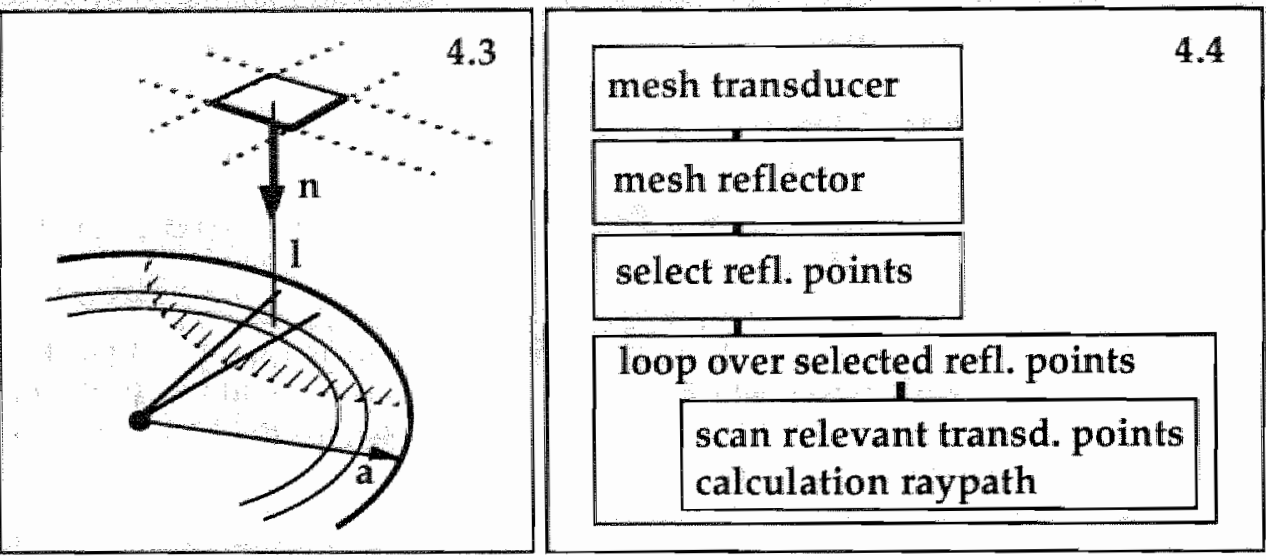

figure 4.3 Elementary reflector with line in the direction of the normal vector $\mathbf{n}$. The region on the transducer for which the rays in the direction of the elementary reflectors return to the transducer is indicated.

figure 4.4 Flowchart of proposed algorithm

By making a loop over all the selected relevant reflector subareas and scanning only the relevant points on the transducer surface for each reflector subarea, the redundant computation of ray paths, that in the end do not contribute, is minimized. A flowchart of the resulting algorithm is shown in figure 4.4 .

\subsubsection{Performance of the algorithm}

In order to evaluate the performance of the proposed numerical method, the calculated Radiation Coupling Function (RCF) of a configuration for which an analytical solution is available was compared with the analytical solution. This analytical solution is given by Rhyne (1977) for the case of a flat circular transducer with a uniform amplitude distribution in combination with a flat parallel plane as a reflector. The analytical solution for a plane reflector at distance $d$ from the transducer with radius a is given by:

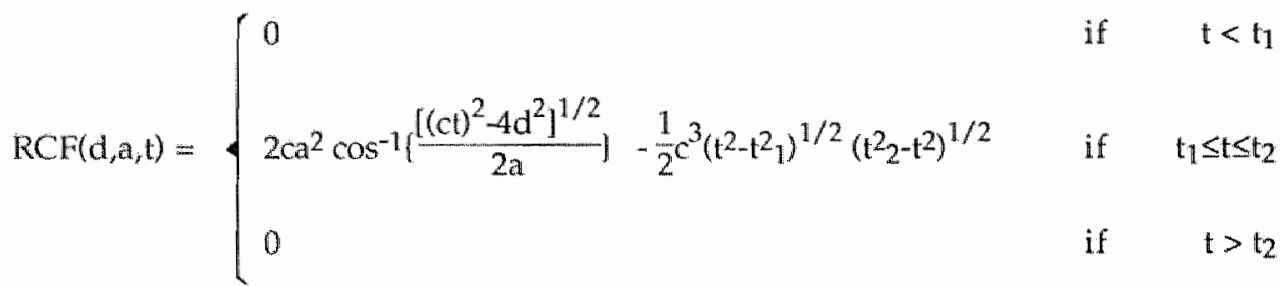



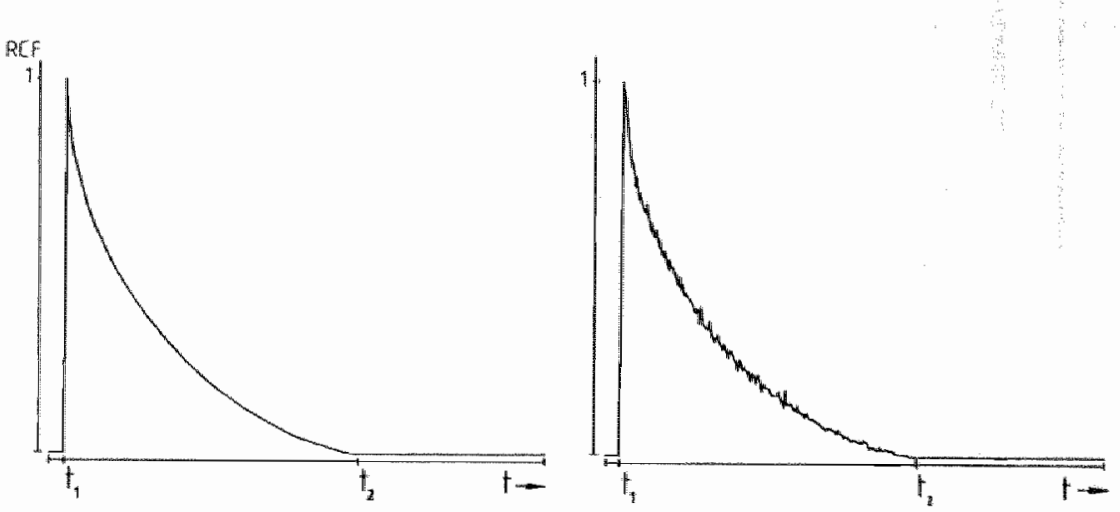

figure 4.5

Analytical solution according to equation 4.19 (smooth curve) and the result of the proposed numerical procedure (noisy curve). Both curves are scaled with respect to their peak value.

with $t_{1}=2 d / c$ and $t_{2}=\left(\frac{4 a^{2}+4 d^{2}}{c^{2}}\right)^{1 / 2}$. The value of $a$ is $3.175 \mathrm{~mm}, d$ is $15 \mathrm{~mm}$ and $c$ is $1484 \mathrm{~ms}^{-1}$.

In figure 4.5 the analytical solution according to equation 4.18 is compared to the results of the proposed numerical procedure. It can be seen that the algorithm performs well. Apart from a scale factor there is only numerical noise due to the temporal and spatial sampling (as discussed in the following section). Another property of the analytical solution is that the value at $t=t_{1}$ does not depend on the distance between the transducer and the reflector. This result was also found using the numerical approach suggested in section 4.2. Onlly if the number of elementary reflectors was chosen too small, deviations were found (see last paragraph of section 4.2.3.).

\subsubsection{Noise}

Looking at figure 4.5 it can be seen that the calculated RCF curves suffer from numerical errors. There are two causes for this phenomenon. The first is the sampling in the time domain, the second is the spatial sampling on both the transducer and the reflector.

After calculation of the almost exact time of arrival of a ray, the contribution from that ray (equation 4.13) is attributed to the preceding sample point. However, in the vicinity of a discontinuity large errors may occur if the amplitude of the real RCF curve changes rapidly within the time interval $\Delta t$. The result of choosing too large a sampling interval is shown in figure 4.6. The computation time is not influenced by the choice of the sampling interval 

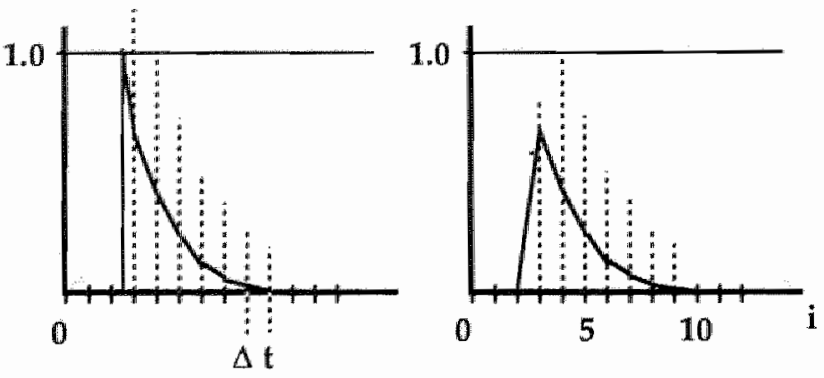

figure 4.6 Effect of undersampling (sampling points indicated) of a function with a discontinuity (left panel) on the sampled function (right panel)

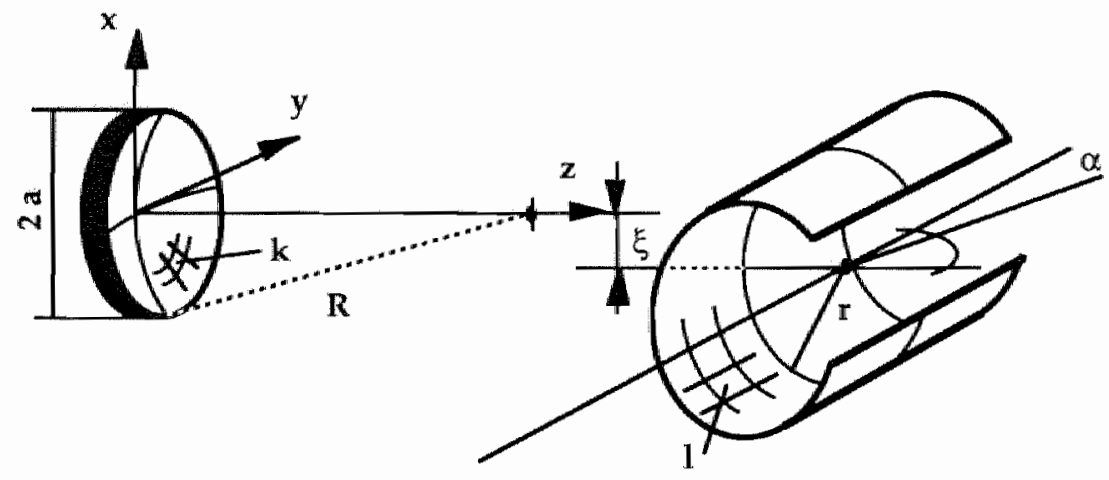

figure 4.7 Geometrical configuration for which RCF curves are calculated. $r=$ radius of cylindrical reflector, $z=$ axial position of the center of the reflector, $\xi=$ lateral displacement. $R=$ radius of curvature of transducer, $a=$ radius of transducer, $\alpha=$ tithing angle.

in the time domain. Therefore, it is possible to choose smaller time intervals in the vicinity of a discontinuily.

The amplitude errors on the smooth parts of the curves are caused by the spatial sampling process. There is only a limited number of combinations of transducer and reflector subareas. Therefore, there is also a limited number of possible traveling distances. The consequence is that the contributions of the source/reflector combinations are not evenly distributed over the sampling points on the time axis. Especially if the distribution of elements on a flat transducer and a flat reflector is the same, a large number of equal traveling distances will be found. The RCF will then have a spiky appearance. Enlarging the number of 

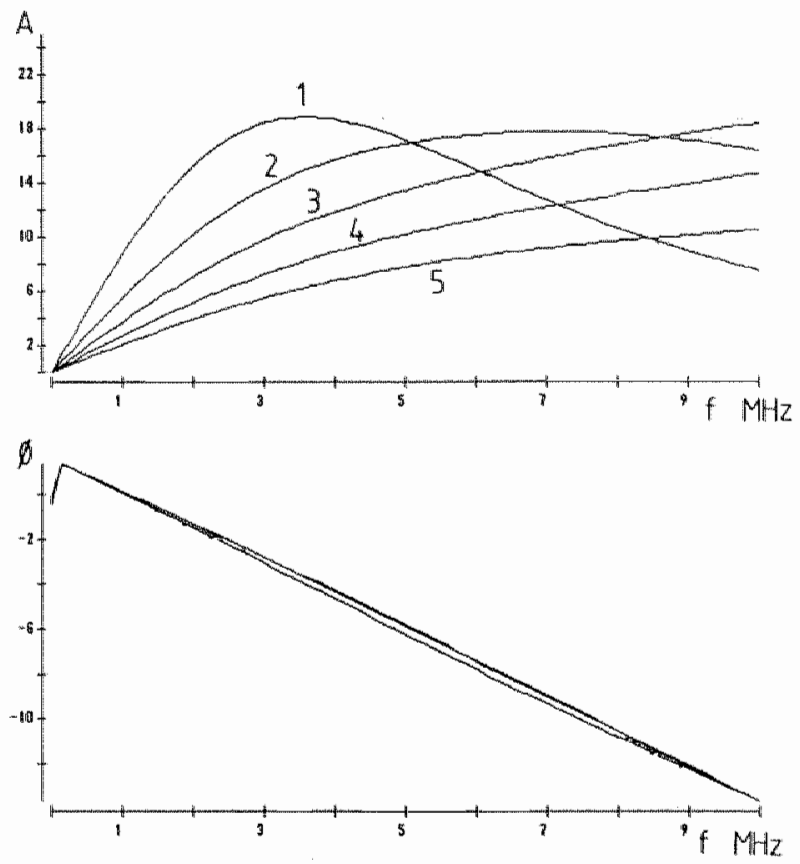

figure 4.8 Influence of axial position of the reflector on the amplitude spectrum of the PCF. The center of curvalure is at $z=20,25,30,35$ and $40 \mathrm{~mm}$ (curves 1 through 5 ). $r=4 \mathrm{~mm}, \alpha$ $=0^{\circ}$ and $\xi=0 \mathrm{~mm}$.

sampling points on the transducer and the reflector will improve the result, but will also lead to longer computation times.

In practica】 situations the problem is not very serious, because most transducers and tissue interfaces are (slightly) curved. The RCF curves for such configurations do not show discontinuities. If the discretizations in both the spatial and the temporal domain are chosen adequately, the spectral components due to numerical noise will be found far beyond the transducer bandwidth, and smoothing of the curves is allowed. Furthermore, the regularity in the traveling distances will be minimal.

\subsection{Results}

As already mentioned in the introduction, the effect of pressure coupling can be regarded as a linear filtering process (see equation 2.10 ). In this section the 

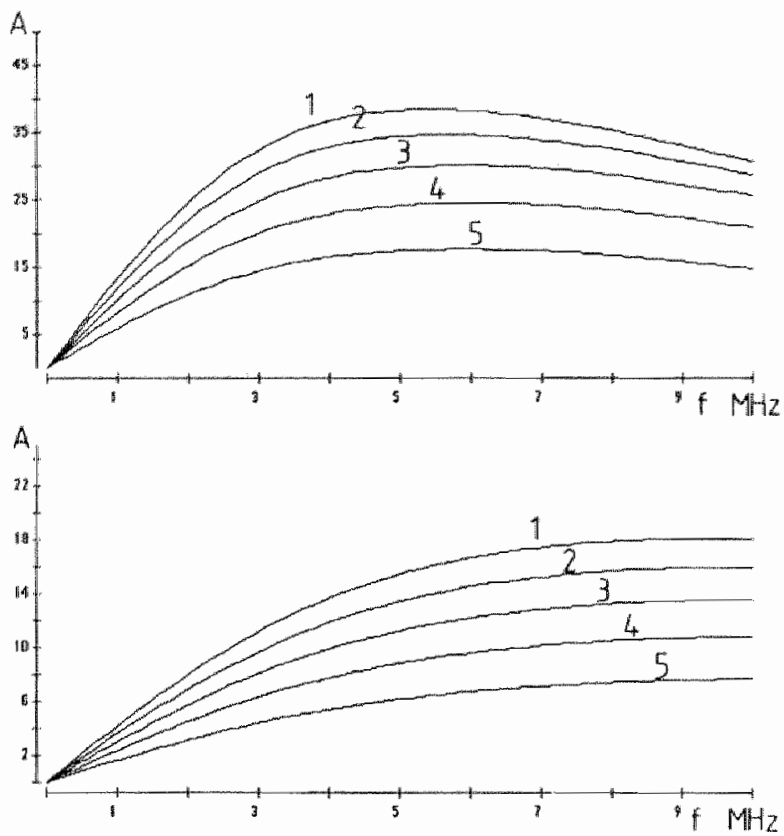

figure 4.9 Influence of the curvature of the reflector on the amplitude spectrum of the PCF. $\mathrm{r}=$ 12, 10,8,6 and $4 \mathrm{~mm}$ (curves 1 through 5). The point of the reflector nearest to the transducer is at $z=20 \mathrm{~mm}$ for the upper and at $z=40 \mathrm{~mm}$ for the lower panel. $\alpha=0^{\circ}$ and $\xi=0 \mathrm{~mm}$ in both panels. (note the difterent scales).

influence of the axial and lateral positions of cylindrical reflectors is studied, as well as the influence of the radius and the orientation of the curvature. The transducer used in the numerical simulations is a circular concave transducer with a non-uniform SVAD. The dimensions are those of a transducer commonly used in the investigation of the carotid artery. The diameter of the transducer is $6 \mathrm{~mm}$, the radius of curvature is $30 \mathrm{~mm}$. The nominal emission frequency is $5 \mathrm{MHz}$. The sensitivity in the reception mode is assumed to be equal to the SVAD. The reflector is a cylindrical surface with the axis perpendicular to the axis of symmetry of the transducer. The radius is $4 \mathrm{~mm}$ in most simulations, which is approximately the radius of the carotid artery (figure 4.7).

The sampling frequency in time is $333 \mathrm{MHz}$. The Fourier transform in equation 4.7 is performed with a Fast Fourier algorithm. Because of the limited duration of the RCF (50-300 sample points), the frequency resolution is in the order of 1-2 $\mathrm{MHz}$. Interpolation is achieved by adding a number of zeroes to the tail of the calculated RCF (up to 4096 sample points). The spectrum of the PCF is found from the spectrum of the RCF by multiplication with jw. From the derivation given in section 2.2 .3 , it follows that the distortion of a reflected pulse is minimal 


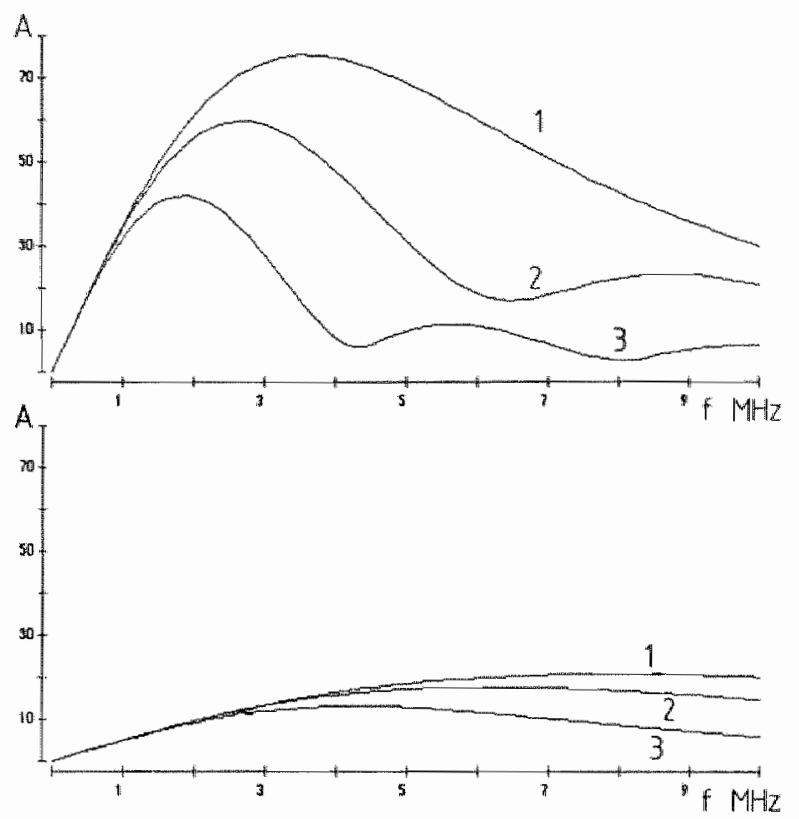

tigure 4.10 Influence of lateral displacement of reflector on the amplitude spectrum of the PCF. d $=0,1$ and $2 \mathrm{~mm}$ (curves 1 through 3 ) $. z=20$ and $40 \mathrm{~mm}$ for the upper and lower panels respectively, $r=4 \mathrm{~mm}, \quad \alpha=0^{\circ}$ and $\xi=0 \mathrm{~mm}$ in both panels.

if the amplitude spectrum of the RCF is flat, or, equivalently, if the amplitude spectrum of the PCF is a linear function of frequency.

The centers of the elementary sources lie on a regular grid of cylindrical coordinates. The number of segments in the direction of the radius is 15 , while in the angular direction there are 60 segments. The largest dimensions are then approximately $0.35 \mathrm{~mm}$. The reflecting surface is divided into rectangular sections, of which the largest dimension is less than $0.1 \mathrm{~mm}$. These dimensions are within the limitations derived in section 2.4.

The frequency response curves are calculated from the unsmoothed RCF curves, as the frequency of the numerical noise $(>50 \mathrm{MHz})$ lies far beyond the frequencies that are of interest $(0-15 \mathrm{MHz}$ or $0-10 \mathrm{MHz})$.

Figure 4.8 shows the influence of the axial position (z-coordinate) of the reflecting surface on the amplitude and the phase as a function of frequency. Amplitude modulation may occur especially if the transducer is situated in the near field or near the focal point (curves 1 and 2). The phase spectrum appears to be almost linear with frequency and the differences between the configurations are marginal. 

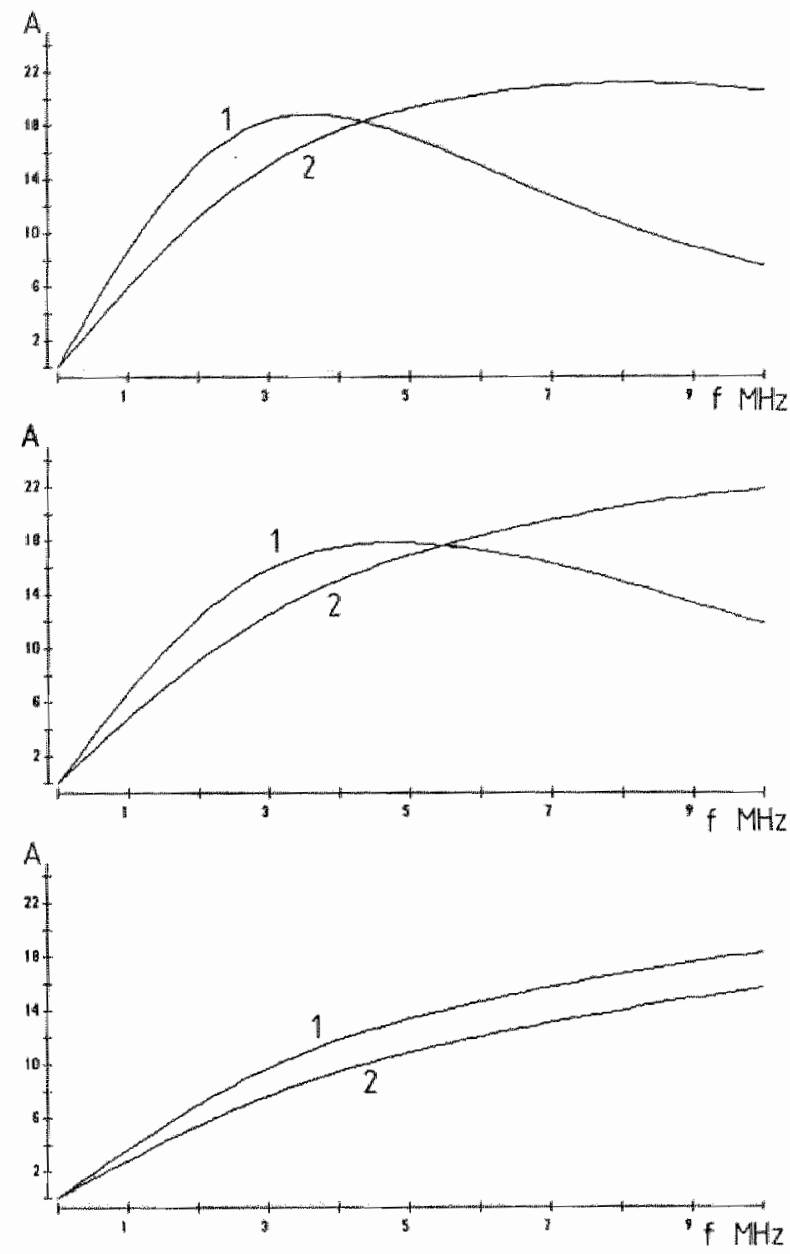

figure 4.11 Difference between the amplitude spectra of the PCF for the anterior wall "curve 11) and the posterior wall (curve 2) of a tube. The axial positions of the center of the tube are 20,25 and $30 \mathrm{~mm}$ for the upper through the lower panels. $r=4 \mathrm{~mm}, \mathrm{a}=0^{\circ}$ and $\xi=$ $0 \mathrm{~mm}$ in all panels.

The influence of the curvature of the reflector is shown in figure 4.9. The dislances to the transducer are $20 \mathrm{~mm}$ and $40 \mathrm{~mm}$, respectively. The most prominent difference between the various configurations is a decreasing amplitude for a decreasing radius of the reflector.

Far more significant are the amplitude spectra shown in figure 4.10. Here the influence of the lateral displacement of a reflector at a distance of $20 \mathrm{~mm}$ is shown. If a cylindrical reflector is not centered on the transducer axis, several frequency components may be strongly reduced ( $>50 \%$ reduction). 

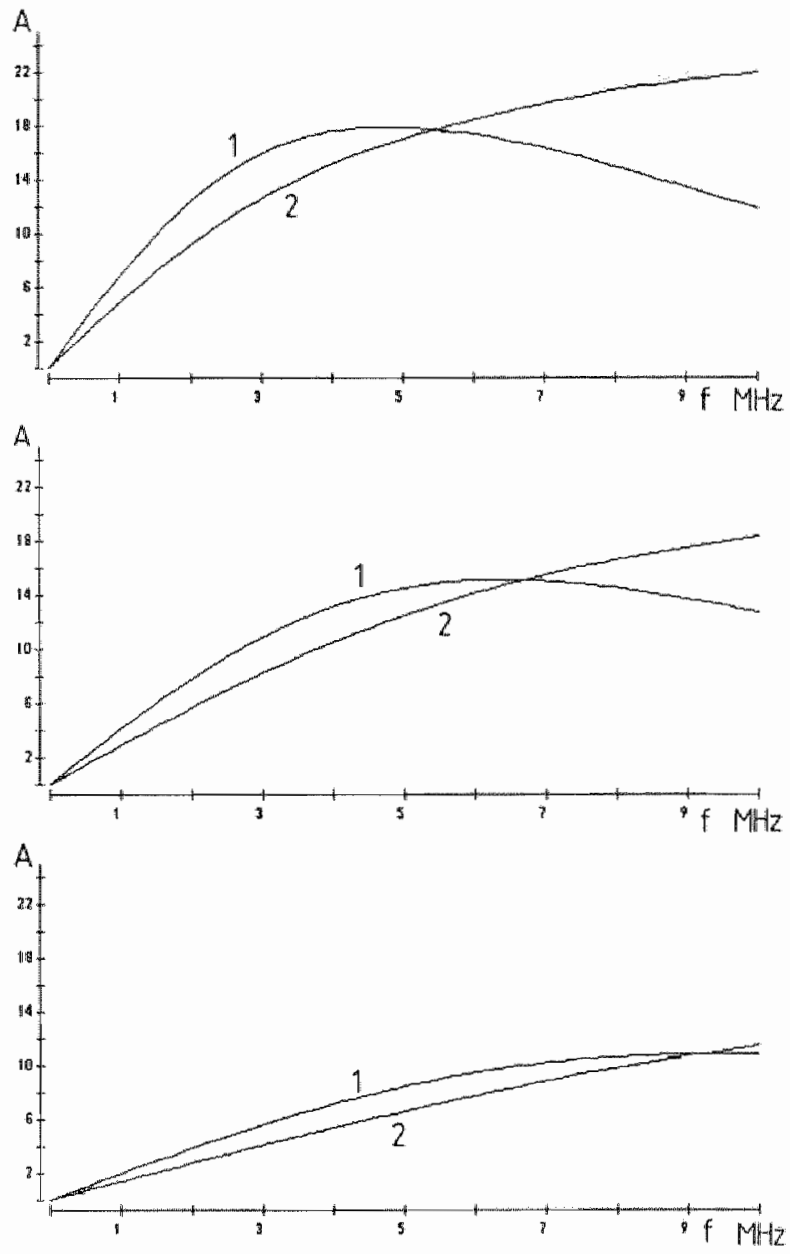

figure 4.12 Influence of angulation of a fube at axial distance $z=20 \mathrm{~mm}$ (anterior wall is curve 1 . posterior wall is curve 2). The tilting angles are $0.0,1.43$ and 2.86 degrees for the upper through the lower panels. $r=4 \mathrm{~mm}$ and $\xi=0 \mathrm{~mm}$ in all panels.

The transducer model is that of a transducer commonly used in the investigation of superficial arteries. Figure 4.11 shows the amplitude spectra of the reflections of the posterior and anterior wall of such a cyllindrical tube (e.g. artery). The axial distances are 20,25 and $30 \mathrm{~mm}$ for figures 11a through 11c. The first two configurations are in the near field of the transducer and clear differences can be observed between the curves of the anterior (curve 1) and posterior walls (curve 2). In figure $4.11 \mathrm{c}$ the center of the tube is in the focal point and mainly an amplitude scaling is observed. 
The effect of an angle of less than 90 degrees between the axis of the tube and the axis of the transducer is showm in figure 4.12 . The axial distance is $20 \mathrm{~mm}$ (point where the two axes intercept). Even a slight deviation from a perpendicular configuration results in great differences in frequency content of the received echoes.

The PCF of most transducer/reflector combinations acts as a bandpass filter. The width of the bandpass is inversely proportional to the effective duration of the RCF. Only if the RCF has a discontinuity the reflection process will have the highpass characteristics described by $\mathbb{R}$ hyme (1977). It should be noted that there are great differences in the Pressure Coupling Functions for the different configurations within the frequency band used in medical transducers (in this case $3.5-7.0 \mathrm{MHz}$ ). Therefore, great care has to be taken in interpreting echo signals reflected from specular reflectors. Major signal distortions can be expected especially in the pre-focal zone.

\subsection{Discussion}

\subsubsection{Discussion on numerical method}

Apart from some simple geometrical configurations, the influence of the geometry of a reflector on the distortion of a reflected ultrasound pulse cannot be solved analytically. The numerical method proposed in this chapter was found to agree well with the analytical results for a given simple configuration. By taking into account some geometrical considerations it is possible to avoid the computation of redundant information, as may occur in the impulse ray method proposed by Cassereau and Guyomar (1988). Some of the causes of the numerical noise have been discussed, but this numerical noise does not influence the frequency response of the radiation coupling filter in the frequency range of interest.

There are several constraints on the size of the discretization on the transducer and reflector surfaces, imposed by the point source approximation. Violation of these constraints will result in errors.

\subsubsection{Discussion on results}

The deformation of a pulse depends on the characteristics of the PCF in the bandwidth of the pulse. Local minima and maxima result in reduction and enthancement of certain spectral components, respectively. The relation between distortion and bandwidth is illustrated in figure 4.13 . The large variation in the values of the PCF in the bandwidth of the broadband pulse results in a serious deformation. The variation of the PCF in the bandwidth of the narrowband signals is so small that it is allowed to approximate the relevant part of the PCF by a constant. Of course the value of this constant depends on the center frequency of the narrowband pulse. A reflected (sufficiently) narrowband signal can therefore be written as an amplitude scaled version of the emitted waveform (see section 2.4). 

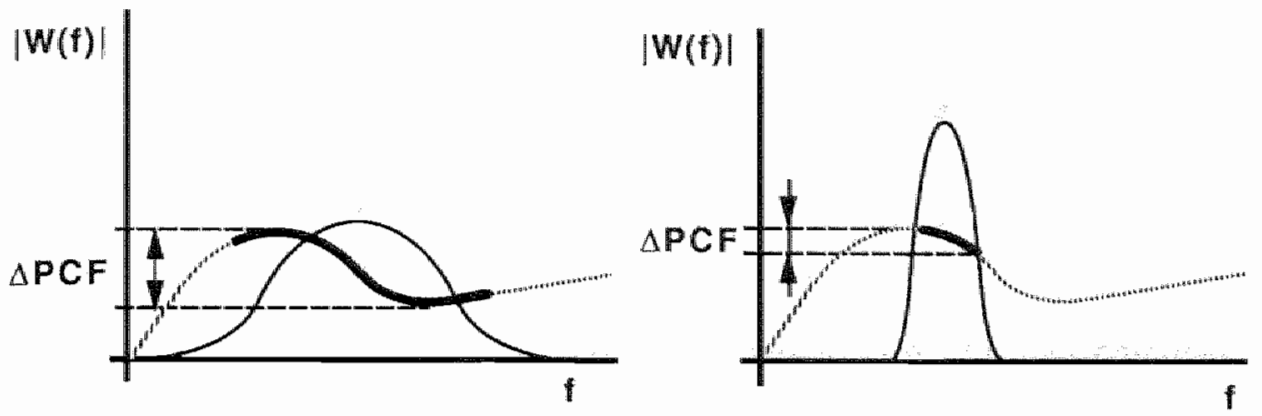

figure 4.13 Distortion due to PCF, for broadband signal (upper graph) and narrowband signal (lower graph). The relevant part of the (fichive) PCF (dotted curve) is indicated by al bolld line.

The results of the calculation of the PCF for several transducer/reflector combinations show that there are major signal distortions, especially within the bandwidth of ultrasound transducers commonly used in medical applications. The frequency content of the PCF of the anterior and posterior walls of a tube (blood vessel) show great differences especially if the tube is situated in the pre-focal zone of a probe. The amplitude spectra of the PCF's of reflectors in the far field of the transducer are much smoother, which means that there is less distortion.

In the far field, the curves have a similar shape, which means that they can be regarded as scaled versions of each other. Variations in the position of the reflector then do not result in a different shape of the reflected signal, but only in a different amplitude.

\section{References}

Cassereau D. and Guyomar D. (1988): Computation of the impulse diffraction of any obstacle by im. pulse ray modelling - Prediction of the signal distortion. J. Acoust. Soc. Amer. 84 1504 -1516

Hottel H.C. and Sarofim A.F. (1967): Radiative Transfer. (Mc Graw.Hill, New York)

Ocheliree K.B. and Frizzell L.A. (1989): Sound field calculations for rectangular sources. IEEE Trans. on Ultrason. Ferroelec and Freq. Control UFFC-36 $242-248$

Penttinen A. and Luukkala M. (1976): The impulse response and pressure nearfield of a curved, ultrasonic radiator. J. Phys. D: Appl. Phys. 9 1547-1557

Rhyne T.L. (1977): Radiation coupling of a disk lo a plane and back or a disk to a disk: an exact solution. J. Acoust. Soc. Amer. 61 318-324

Sung S.H. (1989): Acoustic wave-field analysis of ultrasonic transducers. J. Acoust. Soc. Amer. 86 $1595-1601$

Stepanishen P.R. (1971): Transient radiation trom pistons in an intinite planar, baffle. J. Acoust. Soc. Aner. 49 1629-1638

Verhoef W.A. Cloostermans M.J.T.M. and Thijssen J.M. (1984): The impulse response of a focussed source with an arbitrary, axisymmetric surface velocity distribution. $J$. Acoust. Soc. Amer. 751716 . 1721 


\section{Appendix}

\section{Derivation of maximum dimensions of elementary reflector}

Consider a two-dimensional situation as depicted in figure 4.A1. The rays that have the greatest deviation from the ray traveling between the centers of the lementary source (width $2 \Delta w$ ) and the elementary reflector (width $2 \Delta d$ ) are indicated. The places where these rays hit the transducer surface $(z=0)$ can be calculated as a function of the dimension and the location of the elementary source and the elementary reflector. For low values of $\alpha$ and $\beta, \sin (\alpha)$ and $\tan (\beta)$ can be approximated by their arguments. The expression for the $x$-coordinates $x_{L}$ and $x_{\mathrm{r}}$ is:

$$
\begin{aligned}
& x_{L}=\xi-\Delta d-R \sin \left(2 \alpha-\beta_{L}\right) \\
& x_{T}=\xi+\Delta d-R \sin \left(2 \alpha-\beta_{r}\right)
\end{aligned}
$$

with $\beta_{\mathrm{L}} \equiv \frac{(\xi+\Delta \mathrm{d}+\Delta w)}{\mathrm{R}}$ and $\beta_{\mathrm{r}} \equiv \frac{(\xi-\Delta \mathrm{d}-\Delta w)}{\mathrm{R}}$

The width $2 \Delta r$ of the area where the reflected rays arrive is:

$$
2 \Delta \mathrm{r}=2 \Delta \mathrm{d}+2 \mathrm{R} \cos \left(2 \alpha-\frac{5}{\mathrm{R}}\right) \sin \left(\frac{(\Delta \mathrm{d}+\Delta \mathrm{w})}{\mathrm{R}}\right)
$$

By taking into account that a ray has to return to the transducer surface, the maxinum value for $\alpha$ is approximately $\xi / R$. The maximum value of $\Delta \mathrm{r}$ follows from the restraint that it must be possible to approximate the sensitivity in the reception mode by a constant over the region where the reflected rays arrive. The resulting maximum value can be related to the maximum dimension $\Delta w$ of the elementary sources: $\Delta r \leq k \Delta w$. The maximum value of the reflector can then be found to be:

$$
\Delta d \leq \frac{\kappa-\gamma}{1+\gamma} \cdot \Delta w
$$

Here, $\gamma$ is an approximation for $\cos (\xi / R)$ :

$$
\gamma=1-\left(\frac{\xi}{R}\right)^{2}
$$


In the case of a concave reflector $\Delta \mathrm{d}$ is overestimated by equation 4.A3. For a convex reflector, $\Delta \mathrm{d}$ has to be chosen larger than the calculated value. However, it should be noted that $\Delta \mathrm{d}$ has to be so small that the subarea can be regarded as a flat surface. The value calculated by equation 4.A3 is just an indication.

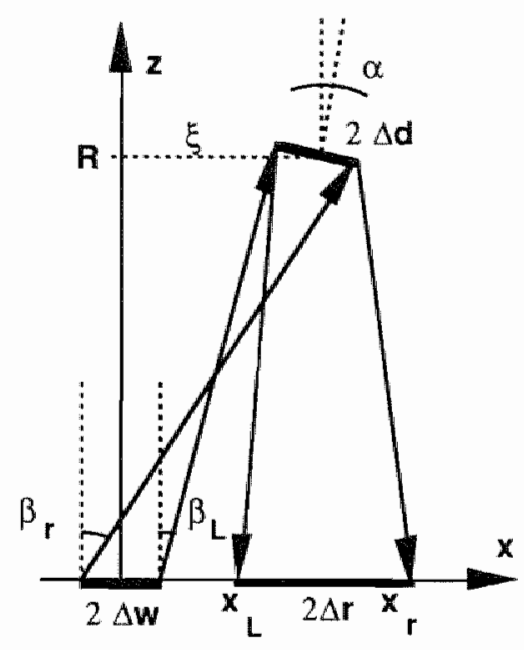

figure 4.A1 Two-dimensional representation of the paths of rays traveling from the edges of an elementary source (width $2 \Delta w$ ) to the edges of an elementary reflector (width $2 \Delta d$ ) and back to the plane in which the elementary source lies. 


\section{NARROWBAND MULTI-FREQUENCY \\ METHOD}

This chapter is based on the publication:

Title: $\quad$ The use of narrowband ultrasound in the characterization of tissue in a thin layer.

Authors: Linssen F.M.J. Hoeks A.P.G. an. Brands P.J.

In:

Phys. Med. Biol. 36 (1991) 1319-1330 


\subsection{Introduction}

Many investigators have attempted to assess the frequency dependence of tissue parameters by analyzing short (broadband) pulses. This is an obvious approach in view of the fact that most ultrasonic scanning equipment uses short interrogating waveforms in order to obtain high axial resolution. Good results were obtained in the assessment of the frequency dependence of the ultrasonic attenuation in such large organs as the liver and the spleen. For such large organs the received echograms have a stationary character. It is then possible to average a large number of spectra of data segments (windows) from different parts of the organ (Kuc and Schwartz 1979, Fink et al 1984, Kuc 1984, see also chapter 2).

This approach is not possible if a thin tissue layer, like that of the carotid artery wall, has to be characterized. In that case only the reflections from the two interfaces are available. If short (broadband) pulses are used the reflections may be separated in time, but a Fourier transform on such a small part of the echogram (Short Time Fourier Analysis, Fink et al 1984) results in a poor spectral resolution and spectral signal to noise ratio (SNR). In this thesis the spectral SNR is defined as the ratio between the peak amplitudle of the spectrum of the signal and the average value of the amplitude of the noise. For example, a 32 - point Fast Fourier transform on a signal, digitized at a sampling rate of $20 \mathrm{MHz}(\sim 1 \mathrm{~mm}$ thick tissue layer), results in a spectral resolution of only $0.625 \mathrm{MHz}$. The accuracy of the data in the frequency domain is not sufficient to resolve small differences in the acoustic parameters at different frequencies (spectral leakage). Also, it is difficult to discriminate between the reflections from the tissue interfaces and the backscatter signal from adjacent tissue, so that selection of the relevant part of the echogram is rather subjective. If there is a signal with non-zero amplitude at the begin or end of the data segment, selection of the window position influences the calculated spectrum obtained by STFA.

To achieve a high spectral SNR with broadband pulses, the amplitude of the signal would have to be extremely large. Not only the decrease in duration, but also the spreading of the power over a larger frequency band would have to be compensated for by an increase in signal amplitude. The instantaneous pressures generated with such a pulse would certainly become larger than what may be considered safe, since the peak pressure depends on the maximum derivative of the emitted pulse form and thus on amplitude and instantaneous frequency (which are both high for such a short pulse). Further, propagation of sound becomes non-linear for large amplitude signals. Apart from this problem, the design of a truly broad banded transducer is very difficult, if not impossible.

Another way to obtain a high spectral SNR may be the use of long, almost monochromatic, pulses. The frequency dependence of the observed phenomena can be assessed by emitting pulses with different central emission frequencies. Instead of one input waveform (electrical input signal), a number of different waveforms is emitted with the transducer in the same position. A separation in frequency of $0.5 \mathrm{MHz}$ can easily be realized, so that the spectral resolution is 
similar to that obtained using Short-time Fourier Analysis (STFA) of broadband signals. This new method will be called the NarrowBand Multi-Frequency method (NBMFmethod). Since the spectral power is concentrated around the central emission frequencies of the different pulses, there, the spectral SNR will improve drastically. There may be a limitation to the energy per pulse due to restrictions on the heating of the insonified tissue, but if the pulse repetition frequency is not too high, a pulse may be elongated without having to drastically decrease the amplitude. However, if the reflecting surfaces are a short distance apart, the reflections of a long pulse will overlap and the temporal (axial) resolution is lost (see figure 5.1). In order to obtain quantitative data for the different reflectors, the influences have to be separated in time again. This can be achieved by deconvolution of the received echogram. After perfect deconvolution, the amplitudes of the peaks in the envelopes of the deconvolved signals represent the reflectivity of the reflections of the different reflectors (tissue interfaces) at the different center emission frequencies.
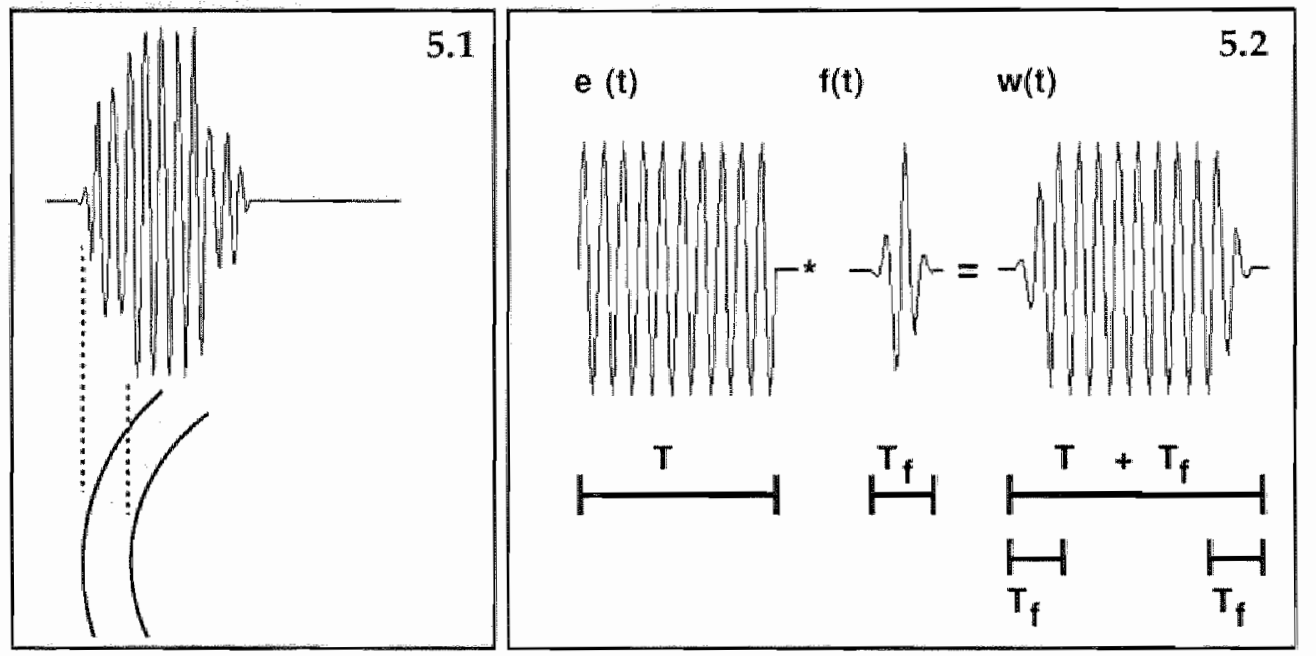

figure 5.1 Simulated reflection of two closely spaced reflectors $\left(f_{C}=7 \mathrm{MHz}, T \sim 14\right.$ periods)

figure 5.2 From left to right: electrical imput signal e(t), overall impulse response $f(t)$, and received waveform w(t) (all simulaled).

The major problem in all deconvolution algorithms is the band-limited character of the emitted waveform (wavelet) and the received signal. In the case of specular reflectors the reflectivity function (see section 2.4, equation 2.17) can be regarded as a series of $\delta$-functions. The amplitude spectrum IR(f) I of such a relectivity function extends over the whole frequency domain. This means that a relatively large part of $R(f)$ cannot be reconstructed from the band-limited spectrum of the received signal. At first sight, this seems to be the major problem in the deconvolution of monochromatic pulses of long duration. However, the high spectral SNR of these waveforms, in combination with correct phase 
information in the frequency band where the spectral SNR is high, provides for a sufficient restoration of the resolution. This is the reason why ordinary Wiener inverse filtering, in combination with a correlation method to reduce artefacts, gives good results for long (narrowband) pulses.

\subsection{Theoretical background: convolution model and deconvolution}

In the narrowband multi-frequency method, as discussed below, the different electrical input signals will be indicated by an index $i$. The individual pulses can differ in center emission frequency and duration (bandwidth). Assuming a single reflector at distance $\zeta_{\mathrm{i}}$, the echo signal refij $(\mathrm{t})$ received upon emission of the $\mathrm{i}$-th pulse can be written as (see section 2.4):

$$
\operatorname{ref}_{i j}(t)=w_{i}(t) * a\left(\zeta_{j,}, t\right) * P C F_{j}(t)
$$

where accents have been omitted for the sake of notational simplicity.

$w_{i}(t)$ is written as:

where

$$
w_{i}(t)=e_{i}(t) * f(t)
$$

$$
f(t)=p_{t}(t) * p_{r}(t)
$$

If $\operatorname{PCF}(t)$ and $a\left(\zeta_{j}, t\right)$ have a smooth and flat amplitude spectrum within the bandwidth of the electrical input signal, the signal $\mathrm{s}_{\mathrm{i}}(\mathrm{t})$ received from a number of reflectors can be written as the summation of the individual reflections (see section 2.4):

or

$$
s_{i}(t)=w_{i}(t) *\left[\sum_{j} C_{j i} \delta\left(t-\tau_{j}\right)\right]
$$

$$
s_{i}(t)=r_{i}(t) * w_{i}(t)
$$

Here, $C_{j i}$ represents the "reflection strength" of the $j$-th reflector at the $i-t h$ frequency. The value of $\mathrm{C}_{\mathrm{j}}$ incorporates the average value of the altenuation function, the average value of the pressure coupling function, and the reflection coefficient for the $j$-th rellector within the frequency band of the $i$-th pulse (see equation 2.15). $\tau_{j}$ is the delay in reception due to the distance between the transducer and the $j$-th reflector. The term in square brackets is the reflectivity function $r_{j}(t)$ and $w_{i}(t)$ is the reflected waveform (often called wavelet in seismological literature). The peaks in the reflectivity function occur at positions where a strong reHector is present. The amplitude of the peak is the reflectivity of that interface at the center emission frequency of the pulse. To obtain $r_{i}(t)$ if $s_{i}(t)$ has been measured, a deconvolution procedure is necessary. It is then assumed that $w_{i}(t)$ is known and $w_{i}(t)$ is called the deconvolution signal. Deconvolution of $s_{i}(t)$ with $w_{i}(t)$ should therefore result in $r_{i}(t)$. For the sake of notational simplicity, the index $\mathrm{i}$, indicating the different electrical input signals used, will be deleted in the following. 
In the frequency domain equation 5.5 is written as:

$$
S(f)=W(f) R(f)
$$

Deconvolution can be easily performed in the frequency domain by dividing the spectrum $S(f)$ of the received signal by the spectrum $W(f)$ of the deconvolution signal:

$$
R(f)=\frac{S(f)}{W(f)}
$$

or

$$
R(f)=S(f) \frac{W^{*}(f)}{|W(f)|^{2}}
$$

where $W^{*}(f)$ is the complex conjugate of $W(f)$. The signal $r(t)$ can be retrieved by inverse Fourier transformation of $R(f)$.

For broad band signals, the shape of the signal will be modified while traveling through the medium, because of the frequency dependence of the acoustical parameters of the tissue. In the case of specular reflections there is also a deformation of the waveform depending on the geometrical dimensions of the transducer and the reflector (Pressure Coupling Function, see chapter 4). The signal modlel according to equation 5.4 is then no longer valid. This is the main reason why deconvolution algorithms have failed to significantly improve the axial resolution of broadband B-mode images (Jeurens et al 1986, Loupas et al 1989, Herment et al 1987). Although determination of the emitted transducer waveform may be possible, this is not a solution to the problem, as the acoustical parameters of the tissue and the geometrical configuration of the transducer and reflector are rarely known in advance. This problem and the problem of the poor spectral SNR can be eliminated by using a narrowband interrogating waveform. This only holds if the pressure amplitude of the emitted acoustical pulse does not exceed the limit for linear propagation.

$w(t)$ is the convolutional product of the electrical input signal e(t) and the overall impulse response $f(t)$ (equation 5.2). If $T_{f}$ is the duration of $f(t)$, and $T$ is the duration of $\mathrm{e}(\mathrm{t})$, it follows from the definition of the convolution that the duration of $w(t)$ is $T+\mathbb{T}_{f}$. The distorted parts (non harmonic) of $w(t)$ are only the first and last $\mathrm{T}_{\mathrm{f}}$ seconds (see figure 5.2 ). This means that the electrical input signal $\mathrm{e}(\mathrm{t}$ ) is a good approximation of $w(t)$ if $T>>T_{f}$. e(t) can then be used as the deconvolution waveform for the whole echogram. For the Wiener-inverse filter there is then no need to correct the measured data for frequency dependent attenuation, diffraction and pressure coupling effects, or to use different deconvolution waveforms for different parts of an echogram. For sufficiently narrowband signals, and smooth attenuation and Pressure Coupling Functions, the peaks in the resulting reflectivity function are proportional to the "reflectivity" $C_{j}$ of the corresponding tissue interface (reflector) (see section 2.4). 


\subsection{Wiener-inverse filtering}

The inverse filter formulation in equation 5.7 or 5.8 will result in large errors if the received signal is disturbed by noise. At frequencies where $W(f)$ approaches zero, the spectrum $s(t)$ will be dominated by noise terms. Division by the locally very small values of $W(f)$ will result in amplification of the noise components. Therefore, a Wiener-inverse filter will be used instead of the inverse filter of equations 5.7 or 5.8. Using $e(t)$ as the deconvolution signal $(e(t) \approx w(t))$, the Wiener-inverse filter for deconvolution becomes:

$$
\left.R(\rho)=\left[\frac{S(\rho)}{E(\rho)}\right] \mid \frac{|E(\theta)|^{2}}{|E(\rho)|^{2}+w}\right]
$$

or

$$
R(f)=S(f) \frac{E^{*}(f)}{|E(f)|^{2}+v}
$$

where $\mathrm{v}$ is the noise cut-off level (Berkhout 1977, Ziolkowsky 1984). This level should be higher than the power spectrum of the noise (within the bandwidth of the emitted signal) to prevent the noise components of the received signal to appear strongly in the deconvolved signal. The first term in square brackets in equation 5.9 is the inverse filtering part, and the second term in square brackets is the Wiener filtering part. From inspection of equation 5.9 it can be derived that the amplitude of $R(f)$ approaches zero if $|E(f)| 2$ is much lower than $v$. Because $v$ is higher than the power spectrum of the noise, amplification of the noise terms is thereby prohibited. An estimate $r(t)$ of the true reflectivity function is obtained after inverse Fourier transformation of the result of equation 5.10. In B-mode imaging, the envelope of the received ultrasound signals is displayed. The envelope of $r(t)$ can be calculated from a Hilbert Transform, which can be readily performed in the frequency domain prior to inverse Fourier transformation.

Because of the narrowband character of the exitation waveform eit $(t)$, the received signal $s(t)$ will also have a narrow bandwidth. It is therefore not possible to calculate $R(f)$ in the whole frequency domain. The result is that the peaks in the envelope of $r(t)$ are not $\delta$-functions (uniform amplitude spectrum in the whole frequency domain), but have a certain width $w$, while side peaks appear as well (see figure 5.3). The influence of the bandwidth of the emitted pulse on the temporal resolution will be discussed in section 5.3.1. The performance of the Wiener filter also depends on the length of the data segment taken into account and on the sampling rate. These effects and the influence of the bandwidth on the accuracy of the peak values in the deconvolved signal will be discussed in section 5.3.2. The side peaks of a large reflection may obscure the main peaks of a nearby weaker reflector. Therefore, section 5.3 .3 will introduce a method by which it is possible to discriminate between real peaks and side peaks and to reduce the amplitude of the side peaks significantly. 


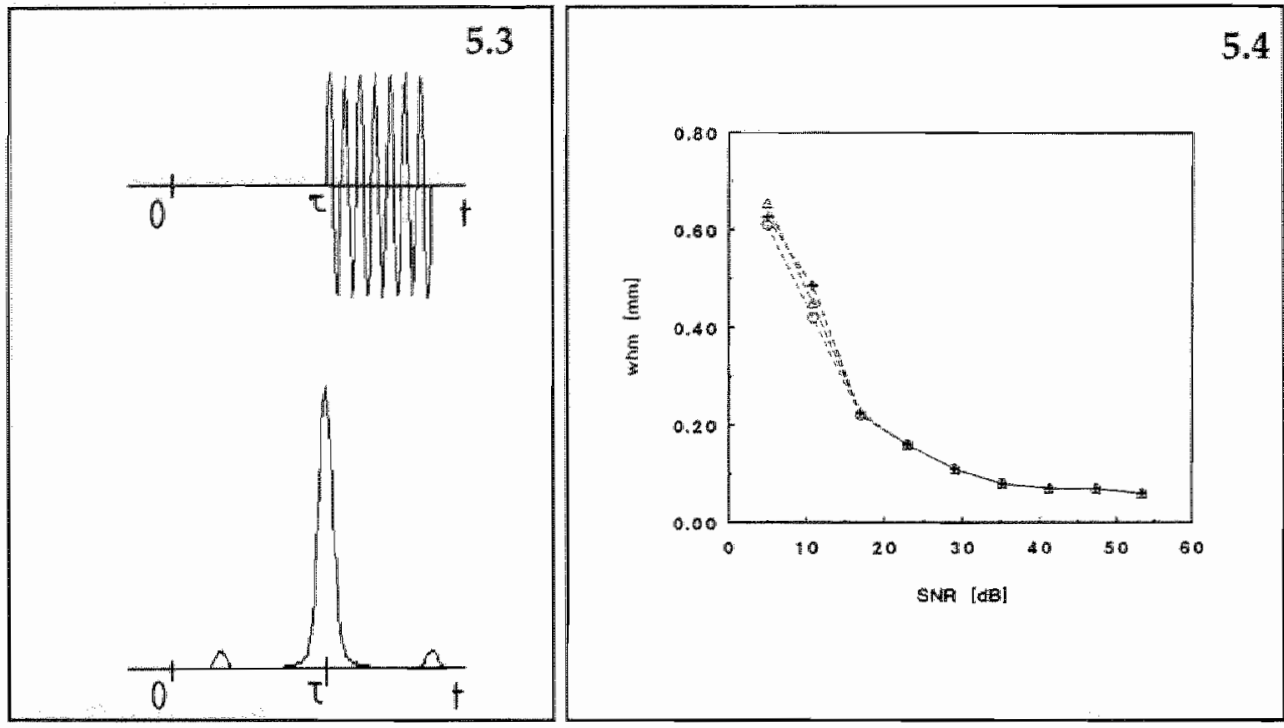

flgure 5.3 Result $r(t)$ (bottom) of Wiener deconvolution of shifted version of electrical input signal e(t- $(t)$ (top).

figure 5.4 Width of the main lobe of a deconvolved signal as a function of temporal RMS ratio of signall and noise. $v$ is equal to spectral RMS of while noise. Pulse duration: $+\sim 1 \mu \mathrm{s}$, $\Delta \sim 2 \mu s, \quad 0 \sim 4 \mu \mathrm{s}$.

\subsubsection{Temporal resolution}

Ideally, the reflectivity function $\mathrm{r}(\mathrm{t})$ is a series of $\delta$-functions (Dirac comb). This can only be realised if the spectrum of $r(t)$ is known in the whole frequency domain (the amplitude spectrum of a $\delta$-function is uniform in the whole frequency spectrum). In practice, $R(f)$ can only be estimated for those frequencies where $S(f)$ has an amplitude which is larger than the noise cut-off level v (see equation 5.9). The result is that the $\delta$-functions are broadened. The width of the broadened peaks (e.g. at half maximum) determines the axial (temporal) resolution. The resolution is the minimum distance (expresssed in travel time or distance) two large reflectors have to be separated before they can be distinguished as separate reflectors in the (deconvolved) ultrasound signal.

The width of the frequency interval, for which the spectral amplitude of the deconvolved signal is significant, determines the temporal resolution. This width depends on the ratio of $\mathrm{v}$ and the power spectrum of the emitted signal. In figure 5.4 the influence of the noise cut-off level on the width of the envelope of the deconvolved signal at half maximum is shown for different lengths of the emitted pulses (1,2 and $4 \mu \mathrm{s}$ ). These pulses are simulated pulses (see section 5.4.1) with white noise added at different levels. The noise cut-off level $v$ is set at the expected value of the spectral power of the noise. This was calculated for a total duration of $s(t)$ of $12.8 \mu$ s or 512 sample points at a sampling rate of $40 \mathrm{MHz}$. The 
temporal Root-Mean-Square (RMS) value of the noise was $\sigma$ and A was the RMS amplitude of the signal in the interval containing a signal.

It is clear from figure 5.4 that a higher Signal-to-Noise Ratio (SNR) (relatively low value of $\mathrm{v}$ or $\sigma / \mathrm{A}$ ) results in narrower peaks. It is also apparent that the duration of the emitted signal (different overlapping curves) does not influence the width of the peaks if the amplitude of the emitted signal remains the same. The amplitude spectrum of a pure harmonic function of duration $T$ is a sinc function. The zeroes of that function can be found periodically at distances from the center frequency which are multiples of $2 \pi / T$. Depending on the spectral SNR a number of side lobes can be taken into account effectively in the deconvolution operation. For the part of the spectrum where $\left|\mathrm{E}(\mathrm{f})^{2}\right|>\mathrm{v}$, the second term in square brackets in equation 5.9 approaches unity. Deconvolution of the corresponding part of $S(f)$ then results in an amplitude spectrum of which the expected value may be approximated by a box function (see figure 5.5). For a box function the inverse transform is a sinc function. The width of the main lobe of that sinc function is inversely proportional to the width of the box in the frequency domain. If the box function is $2(n+1)$ side lobes wide it can be derived that for the width $w$ of the main lobe of the deconvolved function holds:

$$
w \approx \frac{T}{(n+1)}
$$

An increase in duration $T$, results in a decrease of the width of the lobes of the spectrum of $e(t)$. If the amplitude of $e(t)$ is kept the same, however, the amplitude spectrum will increase. This increase in amplitude causes that the width of the frequency interval in which the power spectrum of $e^{(t)}$ is larger than $v$ remains more or less constant. This explains the result shown in figure 5.4, that an increase in the duration of the pulse does not result in a poorer resolution of the deconvolved signal.

Because the spectral SNR depends on the actual length of the recorded signal, it is necessary to window $s_{i}(t)$ in order to increase the spectral SNR. A negative influence of increasing the duration of the emitted signal may be that it is necessary to enlarge the time window, thereby lowering the spectral SNR (see section 5.3.3).

\subsubsection{Data collection and accuracy}

If there is no noise in a stationary signal, the performance of the Wiener filter does not depend on the length of the selected data segment. Normally this is not the case, and it should be kept in mind that the signal power does not necessarily increase if the data segment is longer. The RMS value of the noise in the spectrum will increase and therefore degrade the performance of the algorithm. This leads to the conclusion that the selected part of the echogram has to be as short as possible. Special care must be taken to prevent truncated reflections occurring at the end or the beginning of the selected part of the echogram (see appendix). If this is not possible, a rather large smoothing window should be applied to the end parts of the data segment. 


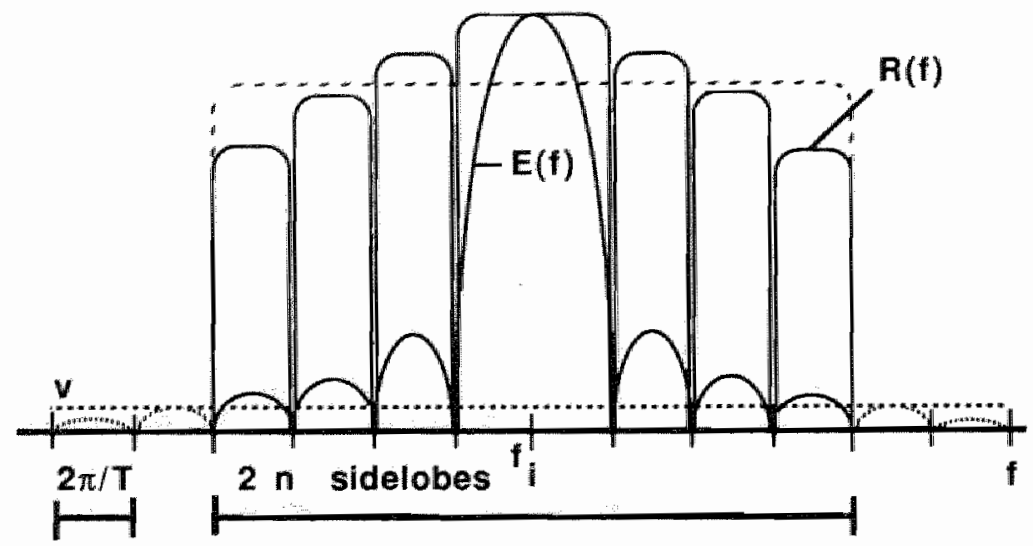

figure 5.5 Amplitude spectrum of a narrowband pulse (time gated harmonic of duration T). If the same spectrum is used for the deconvolution, the amplitude spectrum of the deconvolved signal approximates a box function, with dips at the zeroes of the spectrum of the deconvolution signal.

To prevent noise-aliasing, the sampling rate should be chosen sufficiently high. If this is not possible, a bandpass filter should be applied to the received echograms. The temporal (phase) resolution will also improve if a high sampling rate is used.

As pointed out in the introduction of section 5.3 , it can be expected that the relative influence of the deformed first and last part of a reflection decreases as the duration of the emitted pulse increases. The reason for this is the concentration of spectral power in the parts of the spectrum in which both amplitude and phase information are not distorted by any frequency dependent effects. If there is no limitation on the emitted energy per pulse, it is favourable to choose a pulselength that is twice the thickness of the layered structure. Then the influence of side peaks is always beyond the part of the echogram investigated.

Because of the fact that the acoustic energy in a pulse cannot be increased to an unlimited extent, the amplitude of the signals can be adjusted to the increase in duration. This will result in a decrease of the spectral SNR, and the resolution will become worse. The energy per pulse $\mathrm{E}_{\mathrm{p}}$ is proportional to the duration of the pulse and proportional to the square of the amplitude:

$$
E_{p}-A^{2} T
$$

If the emitted energy per pulse is at its limit, a further increase in duration must be compensated for by an appropriate decrease in amplitude. 


\subsubsection{Detection of side peaks}

As already mentioned, the necessity of a noise cut-off level results in the appearance of side peaks in the deconvolved signal. These side peaks appear at distances from the main peaks that are multiples of the duration of the emitted waveform signal. This is the result of the periodicity of the spectrum of a narrowband pulse: around the frequencies where $|E(f)|$ approaches zero, the amplitude spectrum $R(f)$ is also drawn to zero values (see figure 5.5). This feature, however, can be used to distinguish between the major reflections and the side peaks. By emitting a pulse of the same frequency, but with a larger or smaller number of periods, the main peaks will appear at the same positions after deconvolution. The side peaks, however, will move with respect to their previous positions, and thereby reveal their nature.

A rapid way to reduce the amplitude of the side peaks is by calculating the crosscorrelation value for zero time shift of two deconvolved signals (same center emission frequencies, but different durations of the emitted pulses). The crosscorrelation of the two signals is calculated within a sliding window. The length 1 of the window is approximately one wavelength (at the average frequency within the frequency range used).

$$
R_{r r}(m)=\sum_{k=m}^{m+1-1} r(k) r_{s}(k)
$$

where the index $s$ refers to the result from the shorter pulse. Only the peaks that have not moved will result in large values, whereas the other peaks almost disappear. Because the Hilbert transform $p(t)$ of $r(t)$ is readily available, the same calculation is repeated for $p(t)$ :

$$
R_{\rho \rho}(m)=\sum_{k=m}^{m+l-1} \rho(k) \rho_{S}(k)
$$

From these two signals $\left(R_{\mathrm{rr}}\right.$ and $R_{\rho \rho}$ ) an envelope signal can be calculated. In the presence of noise the values in the region of a side peaks in one of the two signals is multiplied with a noisy signal part in the other signal. The result of the averaging operation (summation term in equations 5.13 and 5.14) will be that the side peaks approach the surrounding noise level. In figure 5.6 a typical result of this method is shown.

\subsection{Testing of the NarrowBand Multi-Frequency method}

\subsubsection{Numerical simulations}

Reflections from specular reflectors were simulated by convolving a harmonic signal (with an integer number of periods) with an impulse response function with a Gaussian envelope. The carrier frequencies used ranged from 3.5 to 7.0 

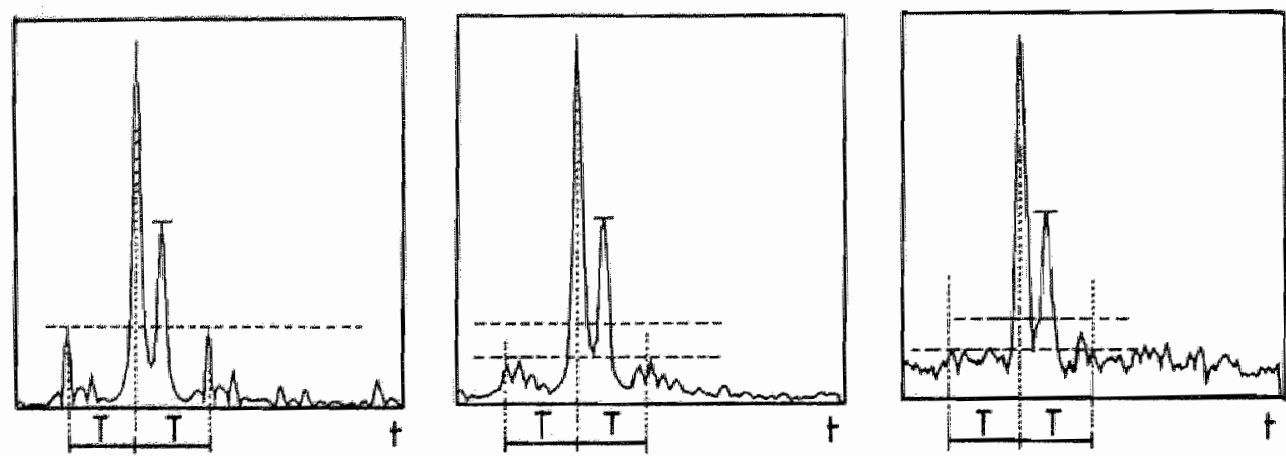

figure 5.6 Effect of multiplying the deconvolution results of signals from emission of pulses with different lengths. The signals consist of 2 simulated reflections with an amplitude ratio of 2 . $\left(I_{c}=5 \mathrm{MHz}_{1} 10\right.$ and 12 periods respectively). Envelope of Wiener-inverse filtered signal of 10-period pulse, no noise (leit). Wiener-inverse filtered result of 10 and 12 period signals correlated, no noise (middle). Same as middle panel, but with noise added in simulated signals (right).

MHz in steps of $0.5 \mathrm{MHz}$. The duration of the pulses was $2 \mu$ s $(80$ samples at a sampling rate of $40 \mathrm{MHz}$ ), which means a duration of 10 periods at an emission frequency of $5 \mathrm{MHz}$.

The impulse response function was created by inverse Fourier transformation of a spectrum with a Gaussian amplitude envelope and zero phase:

$$
|F(f)|=\exp \left(\frac{-\left(f-f_{0}\right)^{2}}{2 \sigma^{2}}\right)
$$

The width of the spectrum was determined by choosing a $\sigma$ of $1.5 \mathrm{MHF}$. The central frequency fo was $5 \mathrm{MHz}$.

Two reflections with different amplitudes and time shifts were added to simulate the signal received from two closely spaced interfaces. The time shif: used was 40 sample points. The amplitude ratio of the two signals was 2.0 in all cases. Noise with a white spectrum was added to the simulated overlapping reflections. The amplitude of the sinulated electrical input signals and the temporal RootMean-Square (RMS) value of the noise were the same for all center frequencies. The temporal Signal-to-Noise Ratio (SNR), as listed in table 5.1, is the ratio between the RMS value of the continuous wave part (middle) of the largest reflection and the RMS value of the noise signal. The differences in Signal to Noise Ratio for the different center frequencies can be calculated from the spectrum of $F(t)$. The value of $v$ was chosen equal to the RMS value of the spectral noise amplitude. The performance of the Wiener-inverse filter was tested by averaging the result of 16 deconvolutions with different noise signals (same temporal power). The results are listed in table 5.1. 


\begin{tabular}{|l|l|l|l|}
\hline $\begin{array}{l}f \\
{[\mathrm{MHz}]}\end{array}$ & $\begin{array}{l}\text { SNR } \\
{[\mathrm{dB}]}\end{array}$ & $\begin{array}{l}\mathrm{Av} \text {. ratio } \\
\mathrm{N}=16\end{array}$ & $\begin{array}{l}\mathrm{SD} \\
\mathrm{N}=16\end{array}$ \\
\hline & & & \\
\hline 3.5 & 9.1 & 2.05 & 0.16 \\
4.0 & 11.5 & 1.90 & 0.10 \\
4.5 & 12.9 & 2.11 & 0.09 \\
5.0 & 13.4 & 1.96 & 0.09 \\
5.5 & 12.9 & 2.12 & 0.13 \\
6.0 & 11.5 & 1.97 & 0.13 \\
6.5 & 9.1 & 2.05 & 0.14 \\
7.0 & 5.6 & 1.93 & 0.14 \\
\hline
\end{tabular}

table 5.1 Average ratio of the peak amplitude values (third column, 16 simulated signals) and standard deviation (fourth column) of Wiener-inverse filtered simulated reflections as a function of carrier frequency. The duration of $e(t)$ was $2 \mu \mathrm{s}$ for all frequencies and the amplitude ratio of the simulated reflections was 2.0 (see text). The temporal SNR (ratio of continuous wave amplitude and RMS-value of the noise) of the smallest reflection is listed in the second column.

As expected, the Wiener-inverse algorithm gives amplitude values which are sensitive to noise. The deviation of the amplitude ratio of the peaks in the Wiener-inverse filtered signals and the true amplitude ratio is rather high (up to $5 \%$ ). Although smoothing of the envelope signal may reduce the random character of the calculated peak values, these peak values still do not represent the actual continuous wave amplitude values at the different frequencies. There seems to be a large bias (appr. 10\%) in the results at the different frequencies, due to the approximation that $e(t) \approx w(t)$ (neglection of the influence of $f(t)$ ). This can be expected, as the spectrum of $r(t)$ becomes wider due to the excellent spectral SNR of the signals. Within such a wide frequency band, the spectrum of $f(t)$ is non-uniform and will therefore have a negative influence on the amplitude of the peaks.

Although there were deviations in the peak amplitude values, the locations of the peaks on the time axis, were quite accurate if the envelope signal was smoothed.

\subsubsection{First in-vivo results}

After building an ultrasound emitter that can emit the desired waveforms, some $\mathrm{M}$-mode pictures of the common carotid artery of a presumably healthy volunteer were made. The emitter was built into a home-made flowmapper (Color Doppler) system in order to be able to see ordinary gray-scale pictures of the region of interest. In the $\mathrm{M}$-mode the ultrasound emitter repeats a freely programmable protocol of four pulses of different lengths. Two pulses were long 


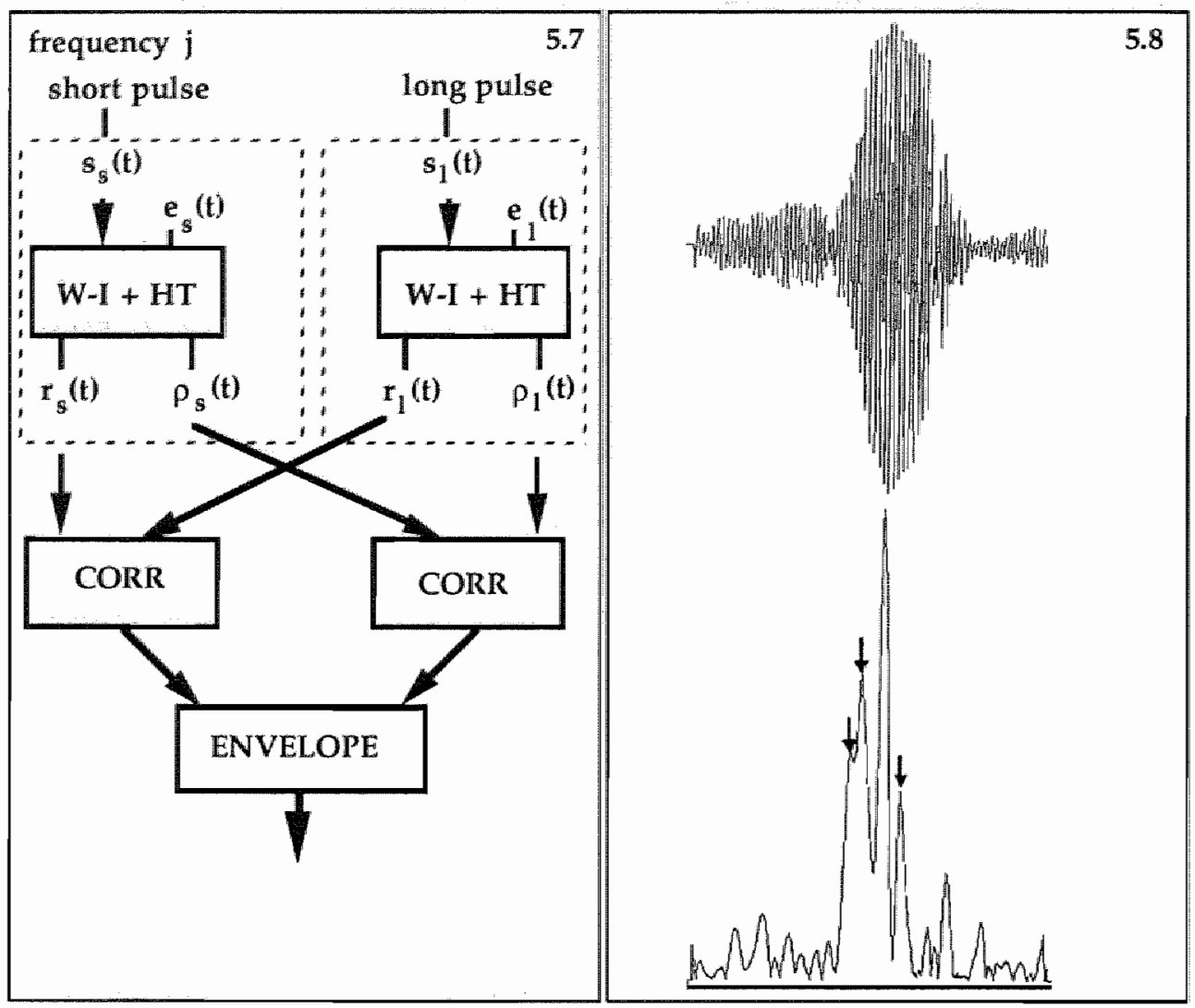

ligure 5.7 Processing of a pair of pulses (long- and short) at the $\mathrm{i}$-th emission frequency. W-1 is the Wiener inverse filtering slep. The results are correllated and eventually envelope detected and displayed. This process is repeated for every emission frequency.

ligure 5.8 AF-signal from anterior common carotid artery wall (in-vivo) ( ${ }^{f} c=6.1 \mathrm{MHz}, \mathrm{fb}_{\mathrm{b}}=42 \mathrm{MHz}$, $T \sim 10$ periods) and result (below) of correlating the Wiener-inverse filtered signal with the Wiener-inverse filtering result of a similar signal obtained from a 13-period pulse. The two pulses are emitted shortly alter each other (PRF $2 \mathrm{kHz}$ )

pulses ( 10 and 13 periods) and two were short pulses (1 period). The emission frequency was $6.1 \mathrm{MHz}$. The RF signals received were transferred to a high-speed data acquisition system, operating at a sampling rate of $42.25 \mathrm{MHz}$. A maximum of 4 Megasamples (10 bit) can be stored and transferred to a mini-computer.

The processing of a pair of narrowband ultrasound signals at one of the emission frequencies is shown in figure 5.7. After data collection, a relevant part of the 


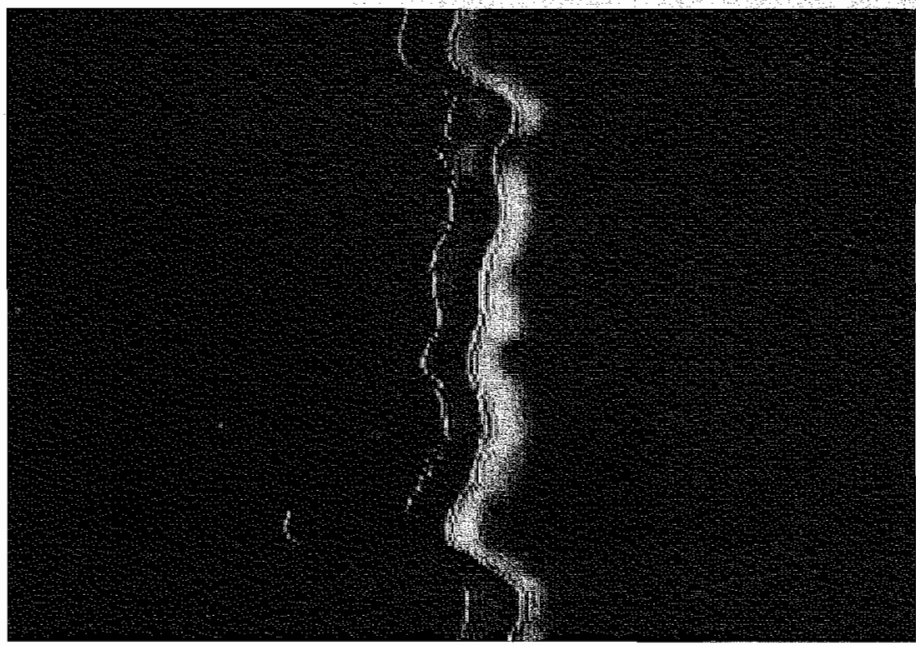

figure $5.9 \quad \mathrm{M}$-mode picture of anterior wall of carotid artery composed trom the Wiener-inverse filltered echo-lines of signals received upon emission of pulses with different length (see also figure 5.7). The positions of the two largest peaks in the decorwolved signal are highlighted.

signals is selected. The signals are Wiener-inverse filtered (equation 5.10) and Hilbert-transformed. The deconvolved signals and the Hilbert-transformed deconvolved signals of the two signals are correlated (equation 5.13). The envelope signal is then calculated from the correlated signals. A typical RF line is shown in figure 5.8, together with the result after correlating two Wiener-inverse filtered signals with the same emission frequency but different lengths. The result is promising, as the typical three-layer structure of the vessel wall appears in the final result. In figure 5.9 the whole $\mathrm{M}$-mode picture of the anterior wall is shown. In this picture the positions of the two largest peaks are marked with bright white points. This picture illustrates a serious problem for the in vivo measurements. Because of the motion of the vessel, the reflectivity of the different tissue interfaces varies too much. One of the causes is the radiation coupling between the transducer and the reflecting structure, which depends heavily on the position of the interface in the sound field of the transducer. Even very small transverse movements of the vessel result in large variation of the amplitude of the different peaks (see chapter 4 ). The result is that the relative amplitude of adjacent peaks may vary. In figure 5.9 this can be seen as sudden jumps of the bright lines to another part of the echogram, because the peaks corresponding to two other tissue layers have then become largest.

\subsection{Discussion}

The amplitude information obtained with the proposed method is far more accurate than that obtained from short time Fourier analysis of the reflections. The main reason for this is the fact that the energy of the emitted pulse is larger than 
that of a short pulse of the same amplitude, and that this energy is concentrated at the central emission frequency. For a short pulse this smaller amount of energy is distributed among the frequencies in the bandwidth of the transducer. The increase in amplitude of a short pulse is limited by the maximum voltage of the transducer (nonlinearity) and also by the fact that the maximum negative pressure must not exceed certain critical values (cavitation). This limitation is more rigid than the limitations on the duration and amplitude of the signal with regard to the possibility of heating the insonified tissue region.

The loss of bandwidth due to the use of a long pulse is compensated for by the fact that the largest part of the energy of that pulse is concentrated in a frequency band where the phase spectrum also allows a good deconvolution. The deformation of the high and low frequency components of a short pulse is the reason that deconvolution of such a waveform does not necessarily work better than the deconvolution of a narrow band waveform.

The transfer function of the echo system causes a frequency and amplitude dependent bias in amplitude ratios (table 5.1). This bias can be partly be corrected for if it is possible to estimate the overall transfer-function. Whether knowledge of the transducer response function can improve the results of Wiener-inverse filtering will be investigated in chapter 6 . Also, the bias will be less if the duration of the emitted pulse is increased. If a thin layered structure is investigated it seems appropriate to choose a pulse length $T$ so that the side peaks occur outside the structure of interest ( $T>2 \mathrm{~d} / \mathrm{c}$, if $\mathrm{d}$ is the thickness of the layer).

The in-vivo results are promising. The fact that the positions of the tissue interfaces can be determined rather accurately opens up a lot of possibilities in the characterization of diseases in the carotid artery wall. The distance between two neighbouring reflecting surfaces may be a useful parameter in the characterization of a pathological process in the tissue between the reflectors. It is also important to know the thickness of the vessel wall if mechanical properties, such as compliance, have to be calculated. It may even be possible to use the information concering the position of the reflecting surfaces to determine the position of RFotracking windows (Hoeks et al 1990) and to investigate the cyclic thickness variation of layers in a vessel wall.

Although the frequency dependent reflectivity of the boundaries of a tissue layer is not only influenced by the tissue in between the boundaries (Chivers and Santosa 1986), it may prove to be a distinguishing parameter for several pathological processes in the artery walls. The problem that the detected amplitude depends heavily on the position of the structure in the sound field, can be reduced by measuring in the far field of the transducer only (see results of simulations in chapter 4). If the image quality is not important, a small transducer with a rather flat and wide wavefront can be used (only the inner disk of an annular array).

The idea of transmitting long pulses can also be of clinical use in the assessment of pathological changes in large organs. Kelly-Fry et al (1988) and Friedman et al (1989) demonstrated the usefulness of frequency specific information in B-mode 
images. Also, the deconvolved A-lines can be used for creating frequency compound images without loss in axial resolution (Trahey et al 1986, Gatenby et al 1989).

\section{References}

Berkhout A.J. (1977): Least squares inverse littering and wavelet decomvolution. Geophysics 42 $1369-1383$

Chivers R.C. and Santasa F. (1986): Numencal considerations for interface rellections in medical uthra sound. Phys. Med. Biol 311 819-837

Fink M. Hottier $F$, and Cardoso J.F. (1984) Ultrasonic signal processing for in vivo attenuation measurement: short time Fourier analysis. Ultrasonic Imaging $5+177-135$

Friedman P.A. Sommer F.G. Chen H.-S. Rachlin D.J. Hoppe F. (1989): Characterization of splenic structure in Hodgkin disease by using narrow-band fittration of backscaltered ultrasound. AuR 152 $1197-1203$

Gatenby J.C. Hoddinot J.C. and Leeman S. (1989): Phasing out speckle. Phys. Med. Biol. 34 16831689

Herment A. Guglielmi J.P. Dumee P. Peronneau P. and Delouche D. (1987): Limitations of ultrasound imaging and image restoration. Ultrasonics $25267-273$

Hoeks A.P.G. Brands P.s. Smeets F.A.M. and Reneman A.S. (1990). Assessment of the distensibility of superficial arteries. Uitrasound in Med. \& Biol. 16 1121-1128

Jeurens T.J.M. Somer J.C. Smeets F.A.M. and Hoeks A.P.G. (1986): The practical significance of Iwo dimensional deconvolution in echography. Ultrasonic Imaging 9 106-116

Kelly-Fry E. Morris S.T. Jackson V.P. Holden R.W. and Sanghvi N.T. (1988): Variation of the transduceir frequency output and receiwer band-pass characteristics for improved delection and image characterization of solid breast masses. Ultrasound in Med. \& Biol 14 Sup. $1143-161$

Kuc R. (1984): Estimating acoustic attenuation from reflected ultrasound signals: comparison of specIral-shift and spectral-difference approaches. IEEE Tr. on Acousfics, Speech and Signal Processing ASSP.32 $11-6$

Kuc R.and Schwartz M. (1979): Estimating the acoustic attenuation coeffichent slope for liver from re. llected ultrasound signals. IEEE Tr. on Sonics and Ultrasonics SU-26 $353-362$

Lim H. Tan K.-C. and Tan B.T.G. (1991): Edge errors in inverse and Wiener filter restorations of motion-blurred images and their windowing treatment. CVGIP: Graphical models and image processing $53 \quad 186-195$

Linssen F.M.J. Hoeks A.P.G. and Brands P.J. (1991): The use of narrowband ultrasound for characterization of tissue in a thin layer. Phys. Med. Biol. 36 1319-1330

Loupas T. Pye S.D. and McDicken W.N. (1989): Deconvolution in medical ultrasonics: practical considerations. Phys. Med. Biol $341691-1700$

Trahey G.E. Alison J.W. Smith S.W. and von Ramm O.T. (1986): A quantitative approach 10 speckle reduction via frequency compounding. Utrasonic Imaging 8 151-164

Zlolkowsky A (1984): Deconvolution. (Dordrecht: Klluwer). 
Appendix

Influence of edge truncation errors

\section{A.1 Cause of edge truncation errors}

The convolution model for the received ultrasound signal $s(t)$ used in the Wiener-inverse formulation is:

$$
s(t)=w(t) * r(t)
$$

where $r(t)$ is the reflectivity function and $w(t)$ is the reflected waveform (note that in the narrowband multi-frequency method this waveform is different for

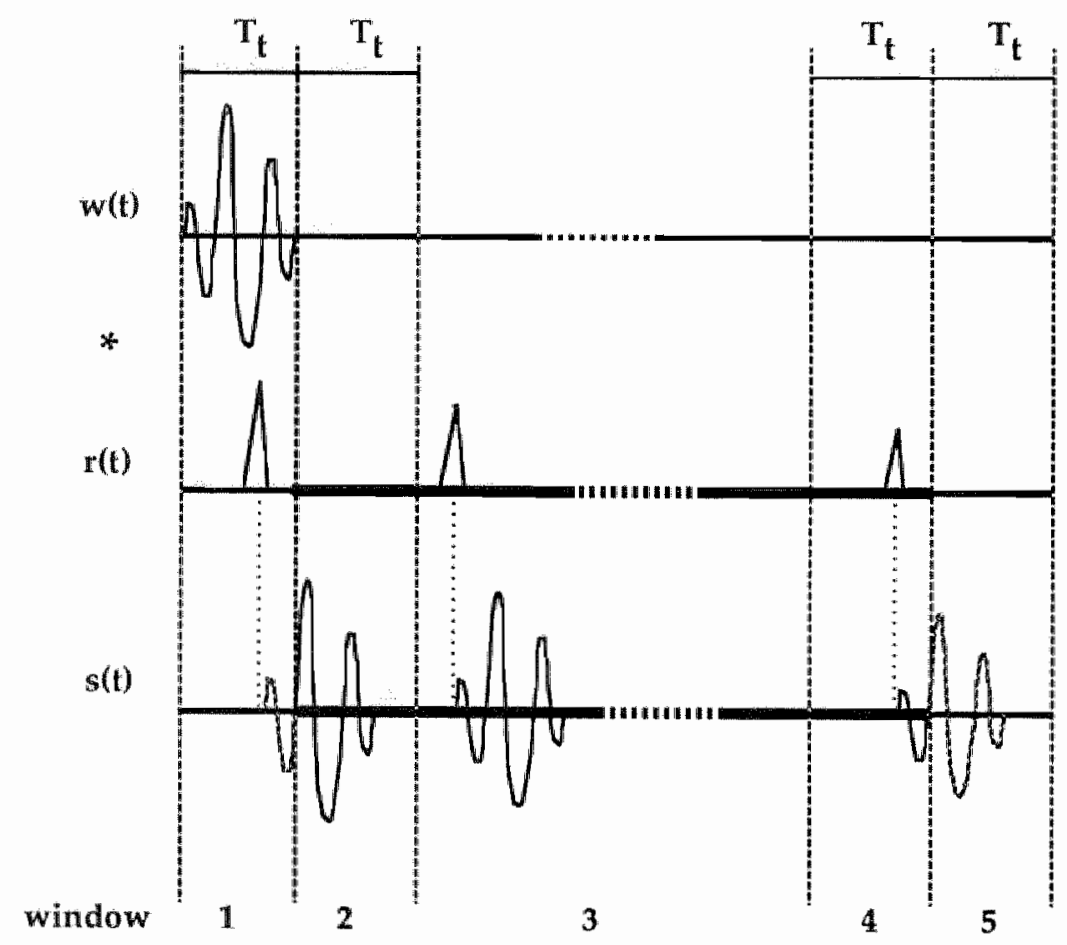

figure 5A.1 Demonstration of causes for edge errors.

Upper signal:

Middle signal:

Lower signal: reflected waveform wit)

reflectivity function $r(t)$. Dirac comb

received signal s:(t) 

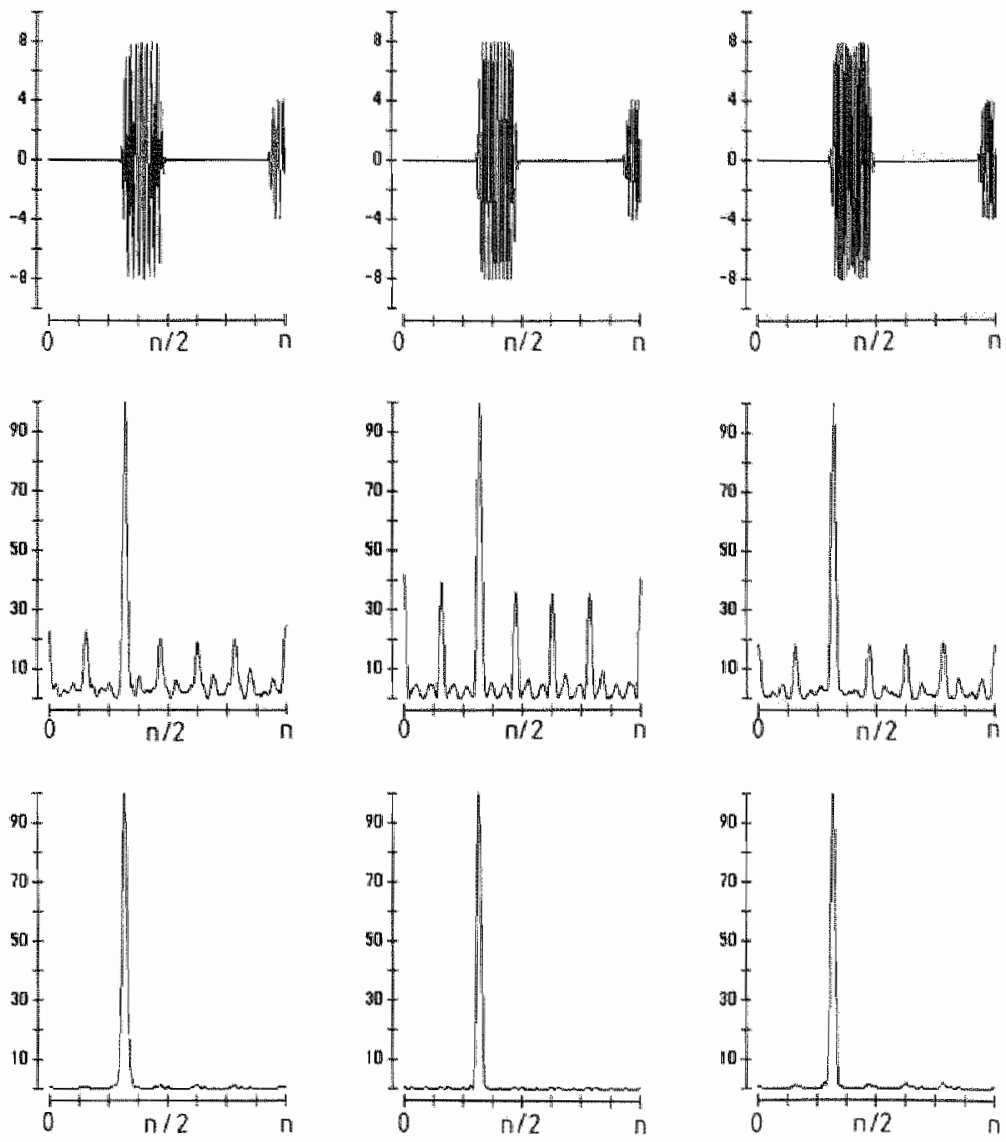

figure 5A.2 Inlluence of a truncated reflection (half the amplitude of the other reflection) at the end of the investigated time window and the result of using a Tukey window to smooth the edges of the input signal. From top to bottom: simulated signal with truncated reflection, envelope of Wiener-inverse fitered signal (no smoothing function) and result of Wiener-inverse filtering after application of a smoothing window to the simulated signal. Vertical axis, scaled such that maximum $=1.0$, horizontal axis: $m=$ 512

each center emission frequency). The inadequacy of the signal model in equation $5 \mathrm{~A} .1$ in the case of truncated echoes is demonstrated in figure 5A.1. The measured part of the ultrasound signal is indicated by the bold part of the time axis. Different time intervals (windows) are indicated by vertical lines. If $T_{t}$ is the duration of $w(t), s(t)$ in window 2 is partly caused by the reflectivity function in window 1. The Wiener-inverse filter, however, does not estimate the reflectivity function in window 1 . A similar problem occurs in windows 4 and 5 . The reflec- 
tivity function in window 4 shoula be reconstructed from $s(t)$ in window 5 , but these are not measured.

In figure 5A:2 the effect of a lruncated reflection at the end of a numerically simulated data segment on the envelope of the Wiener-inverse filtered signal is shown. The simulated data are the same as those used in the simulations of chapters 5 and 6 . The signal is composed of one reflection starting at time sample 100 and a truncated echo starting at time sample 500 of the data segment (512 points). The echo-signals of the $3.5,5.0$ and $6.5 \mathrm{MHz}$ center frequency are shown in the top row (left to right). The middle row shows the envelope of the Wienerinverse filtered signals if there is a truncated echo at the edge of the window. No noise was added to the simulated data.

\section{A.2 Reduction of the influence of edge errors}

The situation that $r(1)$ is zero in windows 1 and 4 would result in a low amplitude of $s(t)$ in windows 2 and 4 . Therefore, a smoothing window function can be applied to reduce the influence of edge errors. Iim and coworkers (1991) derived an "optimal" smoothing window for reducing the edge errors in the restoration of motion-blurred images where the impulse response function is a simple box function. For ultrasound pulses the choice of an optimal smoothing window is less obvious, if not impossible. A simple ramp-function or a cosine-bell shaped smoothing window in windows 2 and 4 may already reduce the effect of edge errors. In the bottom row of figure $5 \mathrm{~A} .2$ the result of applying a Tukey window to the data is shown.

A far better solution is to avoid the edge problem. Now, by choosing a data window with adaptive start and end points, it is often possible to select a part of the signal such that edge errors are greatly reduced. If there is a certain region of interest (e.g. the parts of the signal originating from the anterior and posterior wall of a blood vessel), the starting point of the data window should simply be at a time sample prior to the begin of the region of interest where the amplitude of the signal is lower than a certain treshold. The ending point should be the first time sample following the region of interest where the amplitude of the signal is lower than this treshold.

In the analysis of ultrasound signals, originating from axially scanned blood vessels, there are always parts of the signal with a low reflectivity (lumen of the vessel). Therefore, if the data from the anterior and posterior wall are analysed separately, part of the edge errors can be avoided simply by choosing either the beginning or the end of the data segment within the lumen. All together, the influence of edge errors is relatively small if the begin and end point of the data segments are chosen in a part of the received signal which shows no strong echoes (tissue interfaces). 


\section{CHAPTER 6}

INFLUENCE OF SYSTEM TRANSFER FUNCTION 


\subsection{Introduction}

In the NarrowBand Multi-Frequency method (chapter 5), the received narrowband ultrasound signals are deconvolved using a Wiener-inverse filter. For broadband ultrasound systems (transducer and electronic circuits) in combination with narrowband pulses, the electrical input signal is a good approximation of the reflected waveform. For transducers with a very narrowband transfer function, however, the deviations between the actually reflected waveform wi(t) and the electrical input signal ei(t) may influence the performance of the Wiener-inverse filter. This appeared from numerical experiments, where harmonic functions of different frequencies and integer number of periods were convolved with an impulse response with a Gaussian amplitude spectrum and zero phase (chapter 5). It appeared that the ratio of the peak values obtained by Wiener-inverse filtering of a signal, consisting of two reflections with different amplitudes, suffered from a frequency dependent bias. It is obvious that a frequency dependent bias in amplitude ratios results in errors in the determination of the frequency dependence of acoustic attenuation.

At the $\mathrm{i}$-th emission frequency, the signal $\mathrm{s}_{\mathrm{i}}(\mathrm{t})$ received from the insonified tissue is written as:

where

$$
s_{i}(t)=w_{i}(t) * r_{i}(t)
$$

and

$$
w_{i}(t)=e_{i}(t) * f(t)
$$

$$
f(t)=p_{t}(t) * p_{r}(t)
$$

Here, the reflectivity function $r_{i}(t)$ depends on the center frequency of the emitted pulse $e_{i}(t) \cdot p(t)$ is the system impulse response (both in transmission and reception).

In the NarrowBand Multi-Frequency approach (Linssen and Hoeks 1991; chapter 5) the electrical input signal $e_{i}(t)$ of the $i$-th frequency is used as an approximation of the waveform of a single reflection $w_{i}(t)$ :

$$
w_{\mathrm{i}}(t) \approx \mathrm{ei}_{\mathrm{i}}(\mathrm{t})
$$

This assumption is valid only if the duration of $f(t)$ is very short in comparison with the duration of the $e_{i}(t)$. The reflectivity function is then calculated by inverse Fourier transformation of the result of the following equation:

$$
R_{i}(f)=\left[\frac{1}{E_{i}(f)}\right]\left[\frac{\left|E_{i}(f)\right|^{2}}{\left|E_{i}(f)\right|^{2}+v}\right] S_{j}(f)
$$


Here, the Whener inverse filter is split into an inverse-fitter part and a Wiener flltering part. The first term in square brackets is the inverse filter and the second is the Wiener filtering.

\subsection{Cause of frequency dependent bias in amplitude ratios}

The Wiener-inverse filter is a linear filter, and therefore, the amplitude of a single deconvolved reflection is always proportional to the amplitude of the reflection (except for the influence of noise). The bias in the ratio of the peak values of the Wiener-inverse filtered signal consisting of two reflections must therefore be caused by interference, because the influence of the noise must average out. This means that the envelope of the deconvolved signal at the location of one of the reflections is influenced by the side-lobes of the other reflection. If the energy of the deconvolved signal in the side lobes is different for the different center emission frequencies, the observed bias can be the result. Reduction of this bias is therefore only possible by reducing the energy of the deconvolved signal outside the main peak. Also, this main peak must be as narrow as possible.

If the duration of the electrical input signals is the same for all frequencies, the differences in the Wiener-inverse filter are caused by the overall transfer function. The pulse actually received upon reflection at a reflecting tissue interface can be written as the convolution of the input signall and a distorting impulse response (equation 6.2). The distortion can also be expressed as an additive function to $e_{1}^{j}(t)$ :

or

$$
w_{i}(t)=e_{i}(t)+d_{i}(t)
$$

$$
W_{i}(\mathrm{O})=E_{i}(f)+D_{i}(f)
$$

Because the major part of the energy of the different $\mathrm{E}_{\mathbf{i}}(\mathrm{f})$ is concentrated around the different center emission frequencies, the deformation of $E_{j}(f)$ due to the system transfer function depends on the shape of $F(f)$. In figure 6.1 the deformation of different ei(t) by a Gaussian shaped transfer function is depicted. If the center frequency of $e_{i}(t)$ is the same as that of $F(f)$, the side lobes on either side of the main lobe are uniformly amplifed (niddle panel). If the center frequency of $e_{i}(t)$ is higher, the side lobes left of the main lobe are amplified stronger than those on the right side (right panel). If the center frequency is lower, the opposite effect can be observed (left panel). Because $D_{i}(f)$ is the difference between $W_{i}(f)$ and $E_{i}(f)$, $D_{i}(f)$ therefore depends on the center frequency of the emitted signal. The result is that the level of the side peaks and also the width of the main lobe (resolution) are different for different trequencies.

Another cause for differences in the Wiener-inverse filtering process for different frequencies are differences in the duration of the emitted signals. If the duration of $e_{i}(t)$ is the same for all frequencies used in the narrowband multi-frequency approach, the shape of the corresponding Wiener filter is the same, except for a frequency shift. Very often, however, it is not possible to use the same 


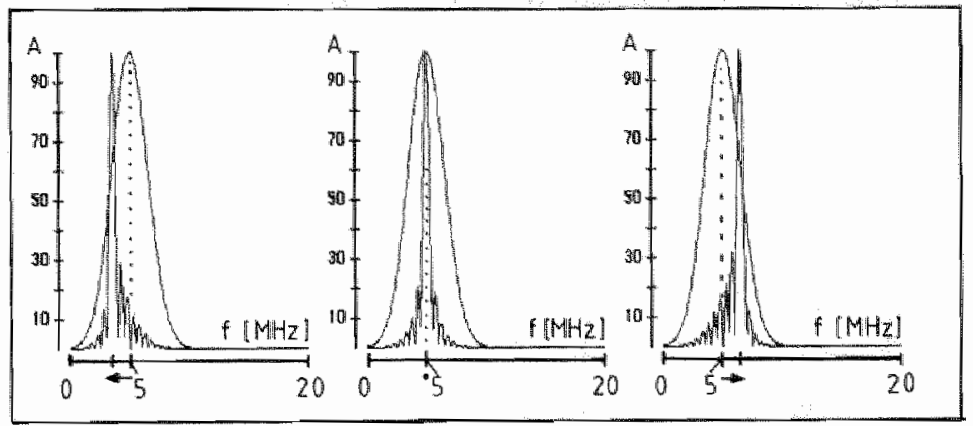

figure 6.1 Deformation of narrowband pulses by a broadband Gaussian transfer function. The Gaussian transfer function is characterised by a mean frequency of $5 \mathrm{MHz}$ and a of $1.5 \mathrm{MHz}$. The spectrum of $2 \mu$ s pulses at frequencies of $3.5,5.0$ and $6.5 \mathrm{MHz}$ are shown after deformation by impulse response function with Gaussian spectrum (left to right's.

duration for all frequencies, because the duration of the pulse has to be chosen equal to an integer number of periods at the different frequencies. The shape of the Wiener filter part then depends on the center frequency of the pulse and hence, the results depend on the frequency. In chapter 5 it was already mentioned, for example, that the distance between the main peak and the side peaks in the deconvolved signal is equal to the duration of the input signal. In the next sections of this chapter it will be investigated whether knowledge of the system transfer function can improve the performance of the Wiener-inverse filter.

\subsection{Improvement by using better deconvolution signals}

\subsubsection{Resolution improvement}

A way to reduce the bias in the peak values of the deconvolved echograms is to use a better approximation of the actually reflected pulses $w_{i}(t)$. The deconvolved signal will then be a better approximation of a Dirac comb, which means that the energy of the signal corresponding to the different reflectors is concentrated in peaks which are as narrow as possible. To obtain a better approximation of $w_{i}(t)$, an approximation $f^{\prime}(t)$ of $f(t)$ is convolved with the different $e_{i}(t)$ which results in a signal $w_{i}^{\prime}(t)$. In equation $6.5 \mathrm{e}_{\mathrm{i}}(\mathrm{t})$ is then replaced by $w_{i}^{\prime}(t)$ :

$$
R_{i}(f)=\left[\frac{1}{W^{\prime} i(f)}\right]\left[\frac{\left|W_{i}^{*}(f)\right|^{2}}{\left|W_{i}^{\prime}(f)\right|^{2}+v}\right] S_{i}(f)
$$

where

$$
w_{i}^{\prime}(t)=e_{i}(t) * f^{\prime}(t)
$$


Detailed knowledge of $w_{i}(t)$ can improve both accuracy and resolution of the Wiener-inverse filtered signals, but in generall $f(t)$ is not completely known (in the appendix a method to estimate $f(t)$ for a real ultrasound system is proposed). In this chapter the influence of deviations in phase and amplitude spectrum will be investigated by means of numerical simulations.
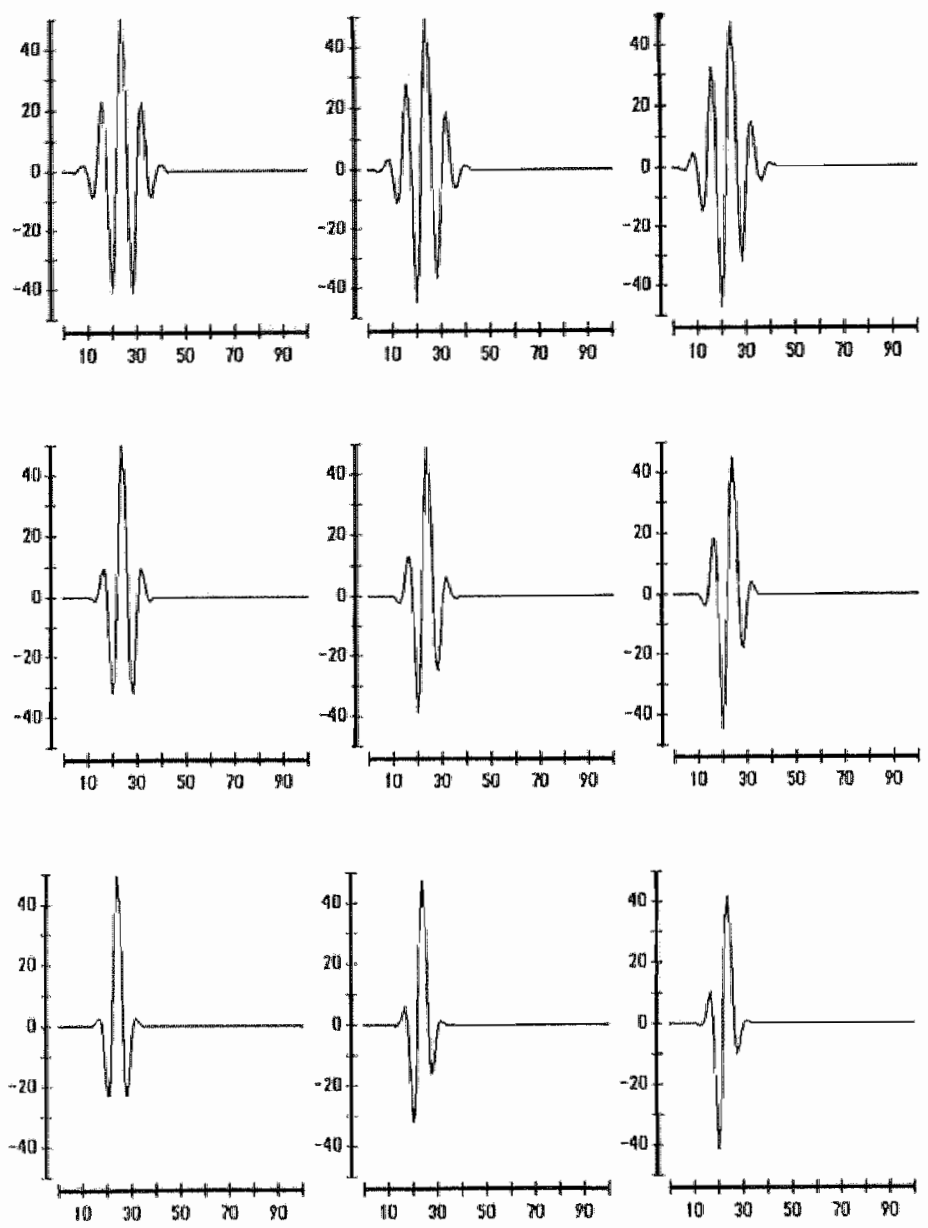

figure 6.2 Time domain representation of Gaussian pulses, all with center frequency $5 \mathrm{MHz}$. The bandwidth is given by the standard deviation of a Gaussian distribution and is equal to $1.0,1.5$ and $2.0 \mathrm{MHz}$ respectively (upper through lower row). On each row, in Irequency domain the phase derivative of the pulse is $0.0,0.157$ and $0.314 \mathrm{rad} \mathrm{MHz}^{-1}$ (from left to right). Vertical axis scaled such that maximum is 50 . Horizontal axis: number of sample points at sampling frequency of $40 \mathrm{MHz}$. 

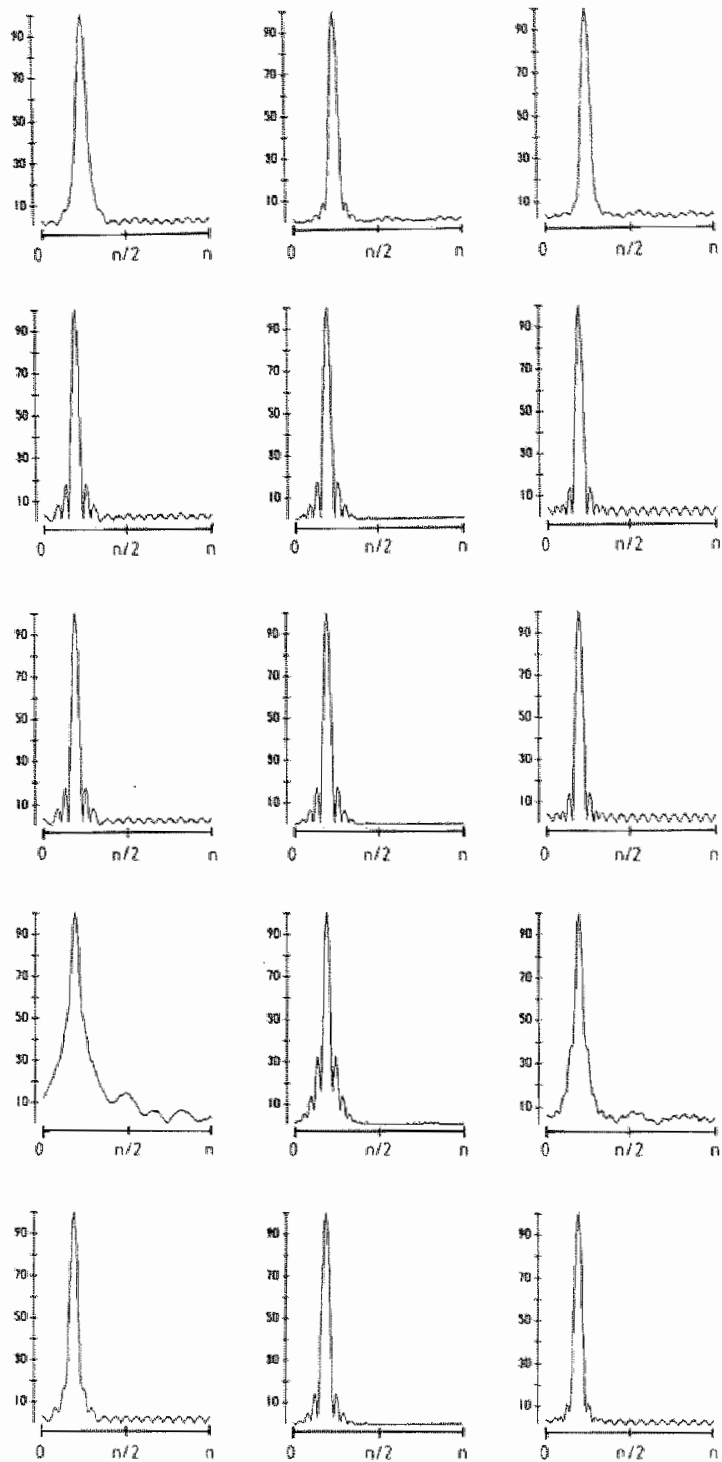

figure 6.3 Result of using different deconvalution signals on the envelope of the Wienerinverse filtered signals. On each row the envelope of the $3.5,5.0$ and $6.5 \mathrm{MHz}$ signals are shown. Vertical axis scaled such that maximum $=100$. Horizontal axis: $n$ corresponds to 512 sample points at a sampling frequency of $40 \mathrm{MHz}$.

- electrical input signal - no correction (row 1)

- correction with Gaussian pulse $f_{\mathrm{C}}=5 \mathrm{MHz}, \sigma=1.5 \mathrm{MHz}$, zero phase, row 2) correction with Gaussian pulse $\|_{C}=5 \mathrm{MHz}, \sigma=1.5 \mathrm{MHz}$, phase correct, row 3) correction with Gaussian pulse $\left(f_{c}=5 \mathrm{MHz}, \sigma=1.0 \mathrm{MHz}\right.$, zero phase, row 4)

- correction with Gaussian pulse $\left\{\mathrm{f}_{\mathrm{C}}=5 \mathrm{MHz}, \sigma=2.0 \mathrm{MHz}\right.$, zero phase, row 5 ) 
To this end different impulse response functions were generated. The amplitude spectrum was always a Gaussian spectrum with a center frequency of $5.0 \mathrm{MHz}$. The shape of the spectrum was determined by choosing different standard deviations $(1,0,1.5$ and $2.0 \mathrm{MHz}$ respectively). The phase was assumed to be a linear function of frequency (see figure 6.2). The phase was zero at the center frequency and the derivative was $0,0.157$ and $0.314[\mathrm{rad} \mathrm{MHz}-1]$, respectively. It can be readily seen that introduction of a frequency dependent phase causes asymmetry of the impulse responses.

Single reflections $(s(t))$ were obtained by convolving the electrical input signals of the different frequencies with the Gaussian pulse with $1.5 \mathrm{MHz}$ standard deviation and $0.157\left[\mathrm{rad} \mathrm{MHz}^{-1}\right.$ ] phase derivative. In figure 6.3 the envelope of one reflection is shown after Wiener-inverse filtering for different deconvolution signals. No noise was added to the simulated reflections, but the value of $\mathrm{v}$ was chosen according to the Signal to Noise Ratios of the simulations in chapter 5 (see table 5.1). On each row the envelope of the 3.5, the 5.0 and the $6.5 \mathrm{MHz}$ signal are presented for different deconvolution signals. The deconvolution signals were either the electrical input signals (top row) or "improvements", obtained by convolving the electrical input signal with one of the different Gaussian pulses (equation 6.9).

If the electrical input signal is used as deconvolution waveform broadening of the envelope of the deconvolved signal can be observed at frequencies other than the center frequency (first row, left and right panel).

The resolution becomes similar for all frequencies if the electrical input signals are convolved with an impulse response function of which only the amplitude information is correct (row 2). There is no significant improvement if the signal is deconvolved with the reflection used as deconvolution signal (perfect deconvolution signal, row 3 ). This result is not surprising as the Wiener-filter part of the Wiener-inverse filter depends only on amplitude information. Hence, the bandwidth of the deconvolved signal depends on the amplitude spectrum rather than the phase spectrum. The only influence of the phase information is on the exact location of the peaks on the time axis. This only holds if the phase is a relatively smooth function of frequency.

The result of assuming a narrowband impulse response is a reduction in resolution (width of main peak at half maximum) at frequencies other than the center frequency (row 4). At those frequencies, the Wiener-inverse filter part then has lower amplitude values than actually allowed, which results in a reduction of the effective bandwidth of the deconvolved signal. The use of $\mathrm{e}_{\mathrm{i}}(t)$ as deconvolution signal implies the assumption of an impulse response of infinite bandwidth. Choosing a broad spectrum is always better than choosing a bandwidth which is too narrow (row 5). 


\begin{tabular}{|l||l|l|l|l|l|l|}
\hline freq. & \multicolumn{2}{|c|}{ peak/signal } & \multicolumn{2}{c|}{$w 1$} & \multicolumn{2}{c|}{ w2 } \\
\hline \multirow{3}{*}{3.5} & \multirow{2}{*}{0.800} & 0.851 & 19 & 13 & 29 & 18 \\
4.0 & 0.823 & 0.863 & 19 & 13 & 27 & 17 \\
4.5 & 0.822 & 0.867 & 19 & 13 & 25 & 17 \\
5.0 & 0.823 & 0.897 & 17 & 13 & 25 & 17 \\
5.5 & 0.806 & 0.875 & 17 & 13 & 23 & 17 \\
6.0 & 0.822 & 0.870 & 17 & 13 & 23 & 17 \\
6.5 & 0.808 & 0.864 & 17 & 13 & 23 & 17 \\
7.0 & 0.773 & 0.858 & 17 & 13 & 23 & 17 \\
& & & & & & \\
& el & r 1 & el & r 1 & el & r 1 \\
\hline
\end{tabular}

table 6.1 The energy concentrated in the main peak as a fraction of the total energy of the deconvolved signal ${ }_{n}$ and the width of the main peak at hall and quarter maximum ( $W 1$ and w2, respectively) for different center emission frequencies (first column). The different deconwolution signals used are indicated at the bottom of each column. el = electrical input signal, $\mathrm{rt}=$ Gaussian pulse (zero phase, $\mathrm{s}=1.5 \mathrm{MHz}$ ). The reflections were simulated using a Gaussian pulse, whith phase derivative equal to 0.157 .

\begin{tabular}{|l||l|l|}
\hline freq. & ratio & ratio \\
\hline 3.5 & 2.09 & 1.96 \\
4.0 & 1.92 & 2.02 \\
4.5 & 2.12 & 2.05 \\
5.0 & 1.94 & 1.96 \\
5.5 & 2.13 & 2.10 \\
6.0 & 1.94 & 2.00 \\
6.5 & 2.08 & 2.07 \\
7.0 & 1.93 & 1.93 \\
sum & 0.69 & 0.39 \\
dev. & & \\
dec-sig & el & r1 \\
\hline
\end{tabular}

table 6.2 Amplifude ratio of two main peaks in envelope of Wiener-inverse filtered signals. The amplitude ratio of the simulated overlapping reflections was 2.0 in all cases. The different deconvolution signals used are indicated at the bottom of each column. el electrical input signal, $r 1=$ Gaussian pulse (zero phase, $s=1.5 \mathrm{MHz}$ ). The reflections were simulated using a Gaussian pulse, whith phase derivative equal to 0.157 . The sum of the absolute values of the deviation from 2.0 is written at the bottom of each column. 


\subsubsection{Amplitude improvement}

To acheve a bettet accuracy of the main peak vallues the amplitude of the side lobes of the deconvolved signals must be as small as possible. In section 6.3 .1 it was already demonstrated that the use of the spectral amplitude information. alone was sufficient to achieve reduction of the width of the main lobe together with a reduction of the side lobes. To demonstrate this, a single simulated reflection was deconwolved using the Wiener-inverse filter. The time interval for which the envelope of the deconvolved signal was larger than half the peak value was determined. The energy of the deconvolved signal in that time interval ("the peak") was calculated as a fraction of the total energy of the deconvolved signal. This calculation was performed on the same signals as in section 3.1 and the results are listed in table 6.1. There, also the width of the main lobe at half maximum (w1) and quarter maximum (w2) are listed. It is obvious that the use of the amplitude spectrum causes a concentration of the signal in the main lobe, where the width of this main lobe also decreases (better resolution). Because the bias in amplitude ratios is probably the result of the interference of the signal in the main peaks with the signal in the side lobes from other reflections, it can be expected that this bias decreases when the spectral amplitude information is used. As an illustration, table 6.2 contains the ratio of two reflections, mixed with amplitude ratio equal to 2.0 , as a function of the carrier frequency. The improvement due to the use of the spectral amplitude information becomes obwious if the absolute values of the deviations from the nominal ratios are summed for all frequencies. For the improved signals this sum is about half the value obtained using the electrical input signals.

\subsection{Improvement of real ultrasound signals}

Narrowband RF signals reflected by a plexiglass rod, immersed in a water tank were oblained using the adapted Color Flow Mapper (see chapter 5). Only the amplitude spectrum of the estimated impulse response function was used. In figure 6.4 the envelope of Wiener-inverse filtered signals of $5.0,6.0$ and $7.0 \mathrm{MHz}$ pulses (2us) are shown from left to right respectively. The envelope signals in the upper row are obtained without using impulse response correction. The envelope signals on the bottom row are obtained using an estimate of the impulse response function using cepstrum filtering of a series of narrowband signals (see appendix). Here, only the amplitude spectrum was used. From comparison of the figures, it appears that the amplitude of the most of the side peaks is lower if impulse response correction is used. Reduction of the width of the main lobe at half maximum, however, can hardly be observed.

\subsection{Discussion}

It would be wrong to condude from the simulations in section 6.3.1 that phase information is not important. Oppenheim and Lim (1981) demonstrated that if only the phase information in the Fourier spectrum is used, and the amplitude is set to unity, the most prominent features of the signals are preserved. They demonstrated this by calculating the two-dimensional Fourier transform of 


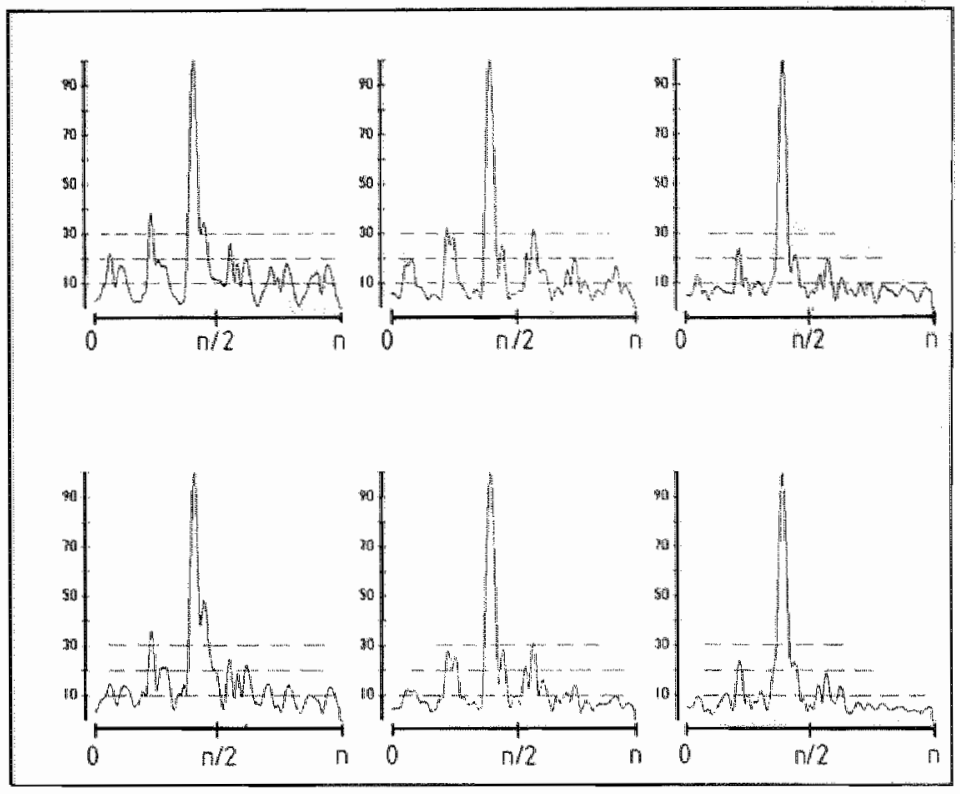

figure 6.4 Result of using impulse response correction on the envelope signall of Wienerinverse filtered signals containing a single reflection from a silicon rod in a water tank. From left to right, $5.0,6.0$ and $7.0 \mathrm{MHz}$ signals are shown. On the upper row, no correction is used. On the lower row, the impulse response function obtained by cepstral filtening is used. Vertical axis, scaled such that maximum $=1.0$. Horizontal axis: $\mathrm{n}$ is equal to 512 sample points in time at a sampling frequency of $42.25 \mathrm{MHz}$.

a photographic image. If only the phase information was used in the inverse transformation, the outlines of the different objects were still visible. The explanation is that the phase spectrum conveys the information about the location of singularities in the time signal. Deconvolution of a time-signal with only one reflection (no noise) can be performed without using amplitude information. Such a deconvolution would imply subtraction of the phase of the deconvolution waveform from the phase spectrum of the signal and setting the amplitude to unity. In a sense, the Wiener filtering part of the Wiener-inverse filter is just a way to select the parts of the spectrum in which the spectrum of the real signal is much larger than the noise signal. The improvement of the resolution for frequencies other than the center frequency of the transducer can be explained by a better weighing of the spectral components.

The use of deconvolution signals which are corrected for the impulse response function of the system is not necessary if the ultrasound system and especially the transducer have a flat amplitude spectrum over a wide frequency range. The development of new transducer materials and the use of new transducer technology may result in great improvements, both for conventional broadband ultrasound imaging and the NarrowBand Multi-Frequency method. 
A special problem is the assessment of the system impulse response of a real ultrasound system. In the appendix, a method based on cepstral bandpass filtering will be presented.

\section{References}

Berkhout A.J. (1977): Least squares inverse fiftering and wavelet deconvolution. Geophysics 42 1369.83

Jeurens T.J.M. Somer J.C. Smeets. F.A.M. and Hoeks APG (1987): The practical significance of two dimensional deconvolution in echography. Ultrasonic Imaging 9 106-16

Linssen F.M.J. Hoeks A.P.G. and Brands P.J. (1991): The use of narrowband ultrasound for characterization of tissue in a thin layer. Phys. Med. Biol. 36 1319-1330

Von Oppenheim A. and Lim J.S. (1981): The importance of phase in signals. Proceedings of the IEEE $69529-541$

Oppenheim A and Schafer R. (1975): Digital signal processing. (Prentice-Hall, Englewood Cliffs)

Robinson E.A. and Treitel S. (1980): Geophysical signal analysis. (Prentice-Hall, Englewood Cliffs) 


\section{Appendix}

\section{Estimation of system tranfer function.}

\section{A.1 Cepstrum domain representation of signals}

In the narrowband multi-frequency method the electrical input signal at the $\mathrm{i}$-th frequency $E_{\mathrm{i}}(f)$ is a narrowband pulse and therefore the corresponding received signal $\mathrm{S}_{\mathbf{i}}(\mathrm{f})$ will also be a narrowband signal. Narrowband signals have an excellent spectral SNR, but only within a narrow frequency band. It is therefore not possible to calculate $F(f)$ over a broad frequency band by means of some deconvolution method. By combining the spectra from narrowband pulses at different central emission frequencies, it may be possible to estimate $F(f)$ over a much broader frequency band. The spectra of echograms received upon emission of pulses at different central emission frequencies from a medium containing a large number of randomly spaced scatterers are added to result in a spectrum $S^{\prime}(\mathrm{f})$ :

with

$$
S^{\prime}(f)=F(f) E^{\prime}(f) \operatorname{Diff}(f)
$$

$$
\begin{aligned}
& S^{\prime}(f)=\sum_{i} S_{i}(f) \\
& E^{\prime}(f)=\sum_{i} E_{i}(f)
\end{aligned}
$$

where $S_{i}(f)$ and $E_{i}(f)$ are spectra of the $i-t h$ frequency and Diff(f) is the (unknown) spectrum of the diffraction function.

$S^{\prime}(f)$ is the product of $F(f), E^{\prime}(f)$ and Diff(f). It is possible to convert this product into a sum of spectra by taking the logarithm of $S^{*}(\mathrm{f})$ (Oppenheim and Schafer 1975):

$$
\log S^{\prime}(f)=\log [F(f)]+\log \left[\mathbb{E}^{\prime \prime}(f)\right]+\log [\operatorname{Diff}(f)]
$$

By taking the inverse Fourier transform equation $6 \mathrm{~A} .4$ is converted to the socalled complex cepstrum domain:

$$
F^{-1}\left\{\log S^{\prime}(f)\right)=F^{-1}\{\log F(f)\}+F^{-1}\left\{\log E^{\prime}(f)\right\}+F^{-1}\{\log \operatorname{Diff}(f)\}
$$

or:

$$
S_{C}(\tau)=P_{c}(\tau)+E_{C}(\tau)+\operatorname{Diff}_{C}(f)
$$

where the index $c$ is used to denote the complex cepstrum representation a time signal. $\tau$ has the dimension of time, but it is called the quefrency in order to stress the difference with the common time domain representation of the 


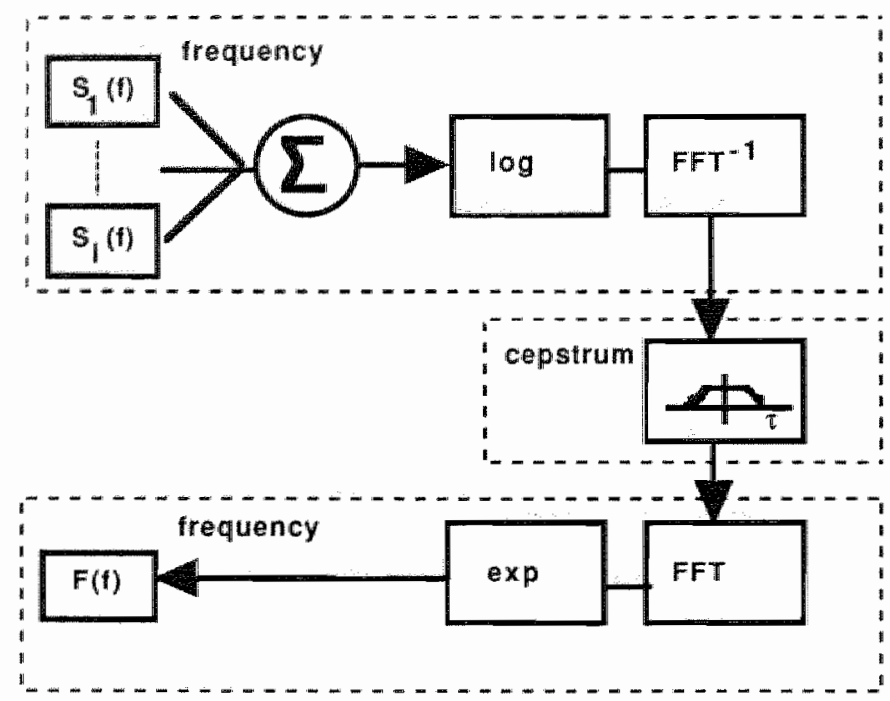

figure 6A.1 Schematic representation of cepstral bandpass filtering. The process starts with the summation of the spectra $S_{i}(\mathrm{f})$ of the signals received at different frequencies and re. sults in an estimate of the spectrum of the system impulse response $F(f)$.

signals. A special problem is the log operation of a complex number. The logarithm of a complex number $x$ is defined as:

$$
\log x=\log |x|+j \phi(x)
$$

where $|x|$ is the complex amplitude of $x$ and $\phi(x)$ is the phase angle of $x$.

Now, if the phase is calculated by an arc-tangent operation on the imaginary and real part, it can be expected that there are discontinuities in the phase spectrum, because the result of this operation is always in the interval $[-\pi, \pi]$. These discontinuities cause errors in the whole cepstrum domain (equation 6A.4). To avoid this, the phase spectrum has to be smoothed by an unwrapping algorithm. The simplest algorithm is the addition of an adaptive constant to the phase calculated by the inverse tangent operation. The value of this constant is adjusted by $+2 \pi$ or $-2 \pi$ whenever the difference between the phase at a certain frequency sample and the phase at the previous sample exceeds a certain treshold value.

\section{A.2 Cepstral bandpass filtering}

For commonly used transducers, $\mathbb{E}(f)$ has a smooth amplitude and phase spectrum. Therefore, in the cepstrum domain, the major part of $F_{c}(\tau)$ is found in a narrow quefrency band around $\tau=0$. The amplitude spectrum of $E^{\prime}(f)$ has two main periodic components. The first periodic component follows from the separation in center frequency $\Delta f$ between the different $E_{i}(f)$. In the cepstrum domain the power of this first periodic component is found in a narrow quefrency band around $|\tau|=1 / \Delta \mathrm{f}$. A second periodic component arises from the narrowband 
character of the different $E_{i}(f)$. This component can be found in the cepstrum domain around $|\tau|=T$, where $T$ is the duration of the narrowband pulses. Because of the noise-like character of Diff(t), the power of Diff $f_{c}(\tau)$ is distributed over the whole quefrency domain.

The influence of $E_{c}(f)$ and $\operatorname{Diff}_{c}(f)$ can then be largely removed from the cepstrum $S_{c}(\tau)$ by bandpass filtering. An estimate of $F(f)$ is obtained by taking the Fourier transform of the bandfiltered cepstrum and inverting the log operation (figure 6A.1).

In order to avoid ringing in the spectral domain after taking the Fourier transform of the bandfiltered cepstrum, it is necessary to use a bandpass filter with smooth edges. A Tukey window is an appropriate window.

\section{A.3. Results}

To estimate the impulse response of the Color flow mapper system an experiment was performed in a water tank. A tissue mimicking phantom, consisting of agar jelly with a very large number of dissolved ferro-carbide particles, was scanned (see figure 6A.2). The phantom was positioned in the far field of the transducer $(30 \mathrm{~mm})$. The region containing the scatterers had very large lateral dimensions which are larger than the lateral dimensions of the ultrasound beam at every frequency used. The ultrasound path in between was water so that there was no significant influence of frequency dependent attenuation effects. The protocol that was emitted consisted of pulses with a frequency between 4.0 and $7.5 \mathrm{MHz}_{x}$ in steps of $0.5 \mathrm{MHz}$. The duration of the pulses was $2 \mu \mathrm{s}$ for all frequencies. Of 64 adjacent echo lines the signals from a region with no specular reflectors were selected and the spectra for all frequencies and lines were added.

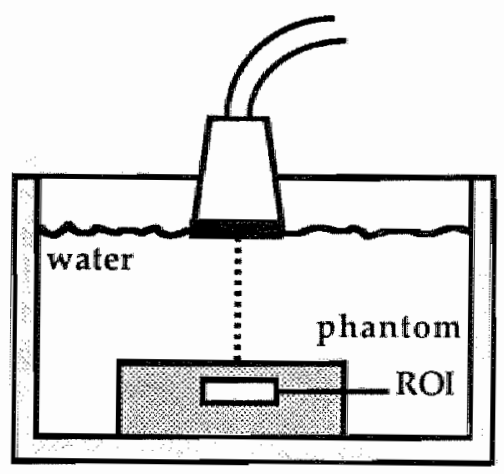

figure 6A.2 Measurement set-up for the estimation of the system impulse response funtion of the Color. Flow Mapper. The signals of which the spectra are calculated originate from a region of interest in a tissue mimicking phantom, immersed in a water lank. 


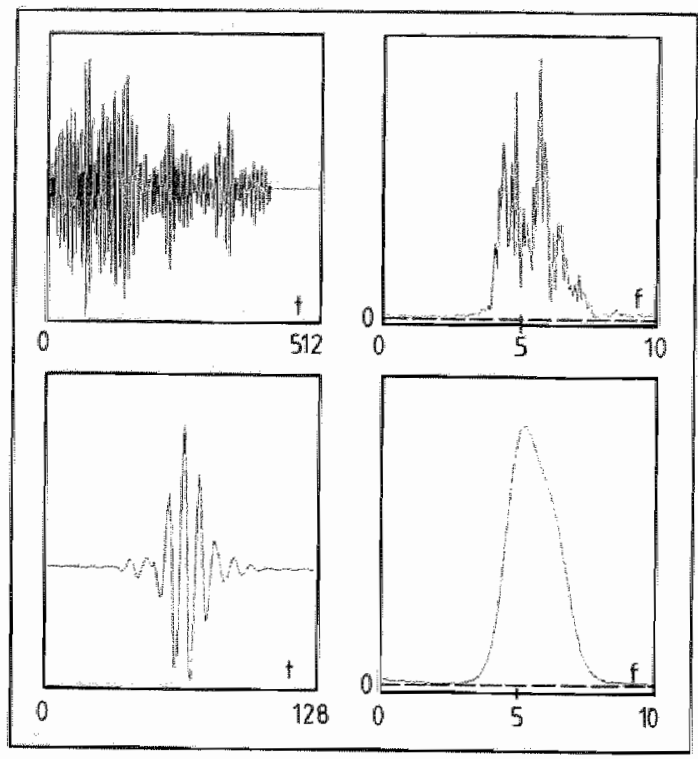

figure 6A.3 Upper left panel: cumulated signal of 64 adjacent A-lines at 9 different frequencies. Upper right panel: amplitude spectrum of signal in upper left panel. Lower right panel: amplitude spectrum of the estimated impulse response function. Lower left panel: estimated impulse response function.

The corresponding time signal is shown in figure 6A.3, upper left panel. The resulting spectrum is shown in the upper right pannel. The amplitude spectrum resulting from cepstral low-pass filtering and the time domain representation of the estimated spectrum of the systems impulse response function are shown in the lower panels (right and left respectively). To allow spectrum amplitude lobes of more than $1 \mathrm{MHz}$ wide, the width of the low-pass cepstrum filter was chosen between -0.5 and $+0.5 \mu \mathrm{s}$. At a sampling frequency of $42.25 \mathrm{MHz}$ this corresponds to a window length of 40 sample points. The up and downslope of the Tukey window were 5 points. Phase unwrapping was achieved using a straightforward algorithm (addition of a constant whenever the absolute value of the difference between two successive samples is more than a certain treshold value (e.g. $\pi$ )). It may be obvious from the appearance of the spectrum of the summed signals, that simple smoothing of this spectrum would result in a very strange shaped spectrum because the interference phenomena are not corrected. The cepstral low-pass filter removes the influence of the interference phenomena and the result is a smooth and continuous estimate of the systems transfer function. 
CHAPTER 7

EVALUATION OF NARROWBAND IMAGING AND PARAMETER ESTIMATION: PRELIMINARY IN-VIVO RESULTS 


\subsection{Introduction}

In chapter 5 the NarrowBand Multi-Frequency (NBMF) method was introduced to assess tissue dependent acoustic parameters while avoiding the problem of the low spectral Signal to Noise Ratio (SNR) normally associated with the use of broad band ultrasound. The major problem in using narrowband signals is the restoration of the axial resolution. In chapter 5 the use of Wiener-inverse filtering was suggested. By correlating the deconvolution result of two pulses with the same centre frequency but of different duration, the artefacts arising from the use of narrowband pulses were reduced. Preliminary results were shown.

In this chapter the performance of the narrowband multi-frequency method will be investigated in in-vivo experiments. An existing Color Flow Mapper system was adapted to allow emission and reception of a protocol of pulses as required by the NarrowBand Multi-Frequency method. Broadband and narrowband echo signals were acquired simultaneously from the common carotid arteries (CCA) of 4 presumed healthy volunteers and 4 patients from the neurological clinic (Academic Hospital Maastricht). These patients suffered from neurological deficits, associated with atherosclerotic lesions in the carotid artery bifurcation, generally in the carotid artery bulb. It will be demonstrated that the method can be used to create high resolution B-mode images. The use of the frequency specific information for assessment of frequency dependent attenuation (tissue characterization) will be investigated, and a method to assess the thickness of the vessel walls will be described.

\subsection{Equipment, data-handling and measurements}

\subsubsection{Equipment}

A Color Flow Mapper system (built in our own laboratories) with a linear array transducer (6.1 $\mathrm{MHz}$ centre frequency, $2.5 \mathrm{MHz}$ bandwidth) was adapted to allow emission of a protocol of different pulses as required by the NarrowBand MultiFrequency method. The transducer is a linear array consisting of 80 elements, spaced $0.30 \mathrm{~mm}$, a group of 16 adjacent elements of which is activated simultaneously (transmission and reception). By shifting the group of activated elements by one element, B-mode images consisting of 64 adjacent A-lines are obtained (see figure 7.1).

The echo data are stored in a 2 Mega-word (10-bit) Data Acquisition System (DAS), operating at a sampling frequency of $42.25 \mathrm{MHz}$. For each echo line the clock driving the sampling circuit is started by the pulse trigger of the Color Flow Mapper. Subsequent echo signals are therefore sampled phase-coherently. Both the Color Flow Mapper and the DAS are controlled by a mini computer (see figure 7.2). The starting point (delay) and the duration of the data acquisition window can be selected in steps of 16 samples. Acquisition of data starts after receiving a frame trigger from the Color Flow Mapper. The first echo signal 


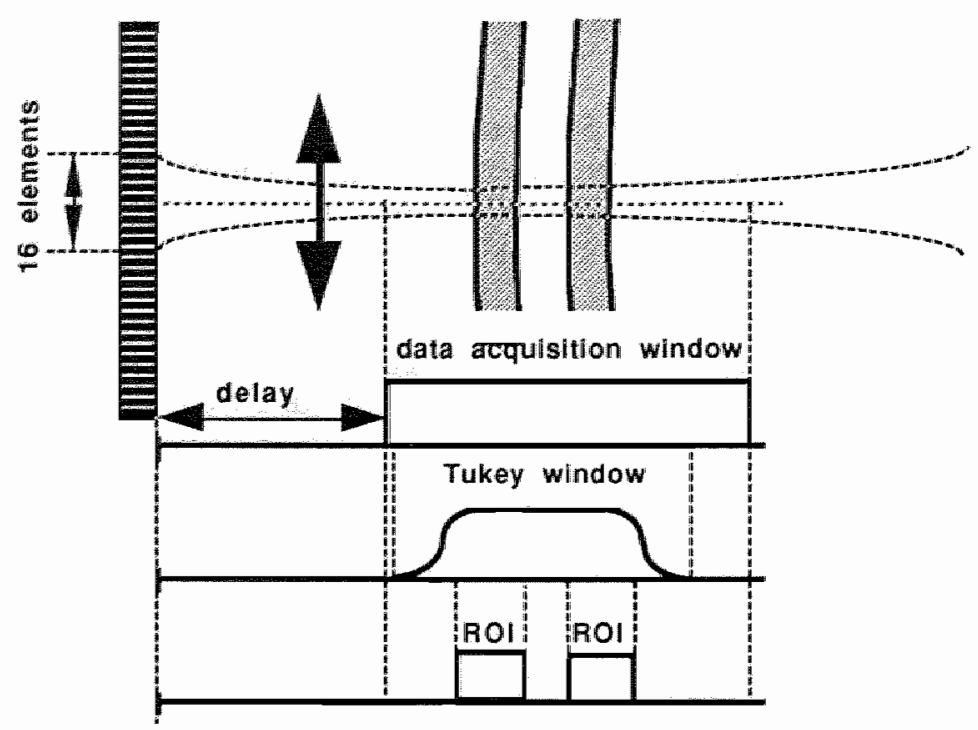

figure 7.1 Linear array transducer with 80 elements. The ultrasound beam is formed by simultaneous activation of a group of 16 adjacent elements. By shifting the group of elements by one element, the beam is translated along the length of the array (arrow). Further, the data acquisition window of the Data Acquisition System is shown. Also, the Tukey smoothing window and the windows used to select the regions of interest are indicated.

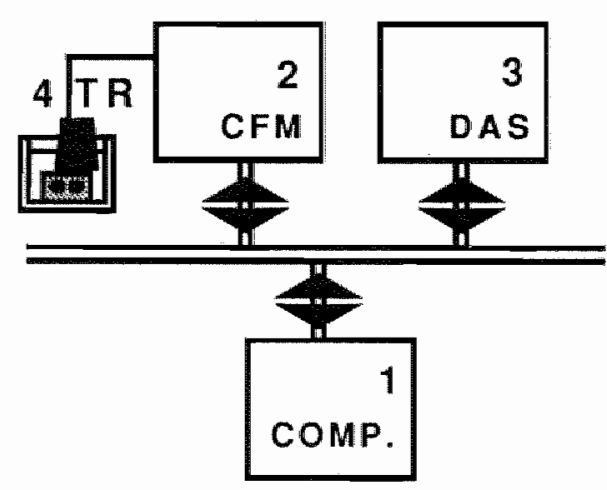

figure 7.2 Set-up of the measurement equipment: $1=$ Mini computer (TORCH Quad-X), $2=$ Color Flow Mapper, 3 = Data Acquisition System, $4=$ Linear array transducer.

The protocol of pulses can be programmed from the mini computer. The Data Acquisition System is Iriggered by the Color Flow Mlapper. The delay in acquisition of the echo lines, the duration of the data window, the pulse repetition frequency, and the sampling frequency can be programmed from the mini computer. After acquisition, the dlata is transterred to the mini computer for further processing. 
stored in the DAS then corresponds to the edge of the imaged region.

At each scan position, a protocol of 16 pulses is emitted before the position is changed (see figure 7.1). The frequency, duration and Pulse Repetition Frequency (PRF) of the pulses of the protocol can be programmed from the mini-computer. If the length of the data acquisition window is 1024 sample points, one image already requires $1 \mathrm{Mega-word}$ of the storage capacity of the DAS: 64 positions, at each position 16 echo signals of 1024 samples each. The organization of the acquired echo data is shown in figure 7.3. A narrowband image is created using the same pair of narrowband pulses from the protocol, at the different positions of the ultrasound beam, which is also depicted in figure 7.3 .

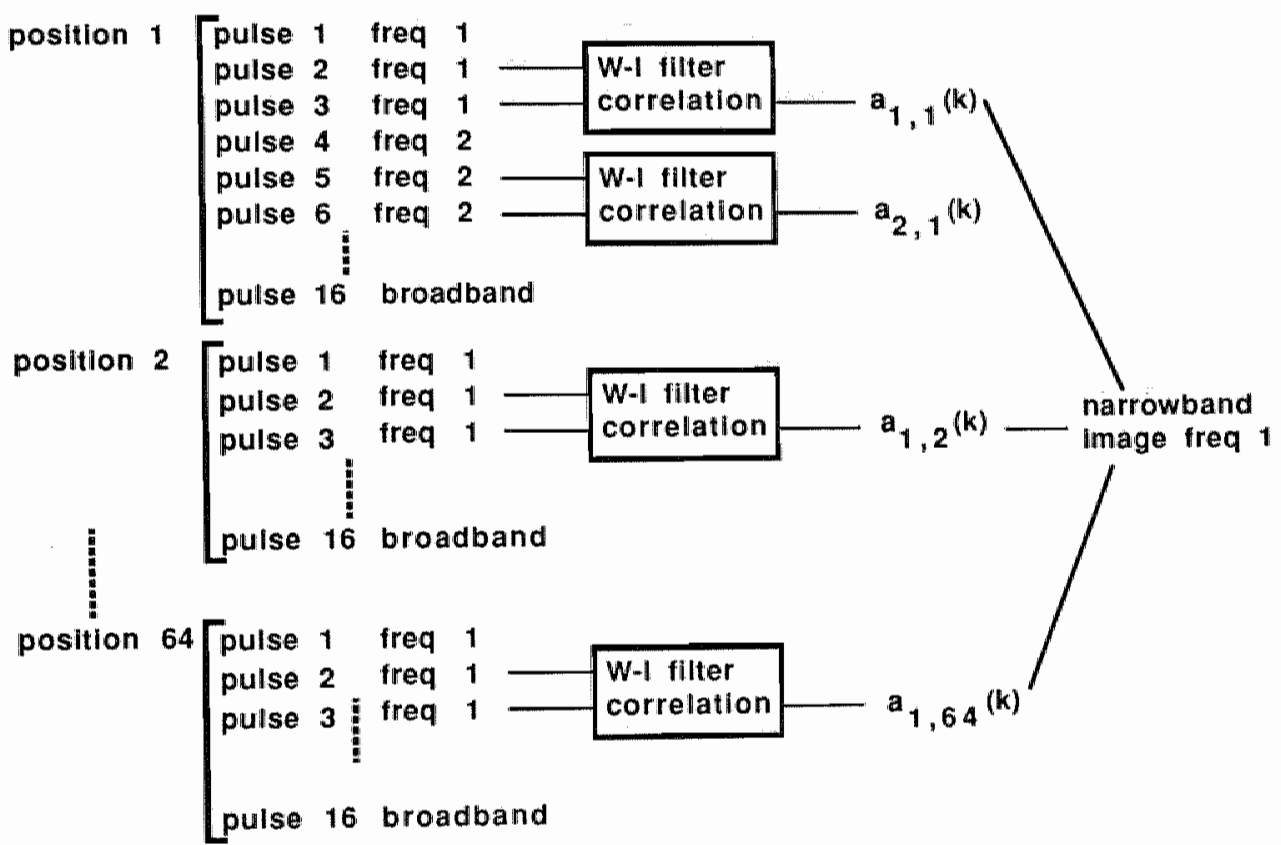

Higure 7.3 Organization of the data in one measurement. A narrowband image can be created by processing the signal received upon emission of the same pair of pulses at subsequent positions of the ultrasound beam. The Wiener-inverse filtering, followed by correlation ol a pair of pulses is illustrated in figure 7.4 .

\subsubsection{Data processing}

The data in the DAS are transferred to a mini-computer for further processing (see figure 7.2). In order to avoid edge truncation errors a Tukey window can be selected in which the echo signals are processed (see figure 7.1). The begin and end point of the window can be selected so that they fall in a part of the received 
signals with a low amplitude (see appendix chapter 5). The up and down slope of the Tukey window both have a duration of $1.5 \mu$ s (which is an average for the pulse durations used). For each frequency a couple of lines is processed as described in chapter 5. After transformation to the frequency domain, the received signals are Wiener-inverse filtered. This is done for two pulses with the same center frequency, but of different duration. To suppress the influence of side-peaks, the results of Wiener-inverse filtering are correlated, where the length of the correlation window is 7 sample points (appr. 1 period at $6.1 \mathrm{MHZ}$, $42.25 \mathrm{MHz}$ sampling frequency).

In addition to the procedure described in chapter 5, it is possible to improve the deconvolution signals (electrical input waveform) by using an estimate of the system transfer function. From the numerical results of chapter 6, it was concluded that the use of only the amplitude information already results in a reduction of the width of the peaks in the deconvolved signals. Therefore, only the amplitude spectrum of the transfer function is used here (zero phase wavelet). The amplitude spectrum of the transfer function was determined with the cepstrum filtering procedure described in the appendix of chapter 6.

The signal processing procedure for a pair of echo lines, indicated by the box in figure 7.3, is further elucidated in figure 7.4. The different durations of a pair of pulses with the same frequency ( $j$ ) are indicated as long (1) and short ( $(s)$, respectively. After correlating both the reflectivity functions and their Hilbert transforms, an envelope signal can be calculated according to:

$$
a_{j, i}(k)=\sqrt{R_{r r i}^{2}(k)+R_{\rho \rho i}^{2}(k)}
$$

where $R_{\mathrm{rr}}$ and $R_{\rho \rho}$ are calculated according to equations 5.13 and 5.14 , respectively, and the index $i$ refers to the line number. The index $j$ refers to the center frequency of the pulses. (see figure 7.4)

\subsubsection{Measurement procedure}

The right common carotid arteries of 4 presumed healthy young volunteers (denoted as Young in table 7.1) and 4 patients from the neurological clinic (denoted as Old in table 7.1) were examined using the NarrowBand MultiFrequency method (see table 7.1). All subjects were males and informed consent was obtained from them all. In two patients the left common carotid artery was additionally examined. The subjects were investigated in the supine position after 5 minutes of rest. The head was slightly tilted towards the contralateral side. The linear array transducer was manipulated in such a position that both the anterior and posterior wall of the artery were clearly visible on the B-mode display of the Color Flow Mapper. The vessel was investigated at an arbitrary site with respect to the flow divider. A second investigator then pushed a button on the Color Flow Mapper to start emission of the pulse protocol and data acquisition. During acquisition of the data, the B-mode image on the Color Flow Mapper was frozen. The measurements were therefore performed blindly. Also, 
it was not known during which phase of the cardiac cycle the measurement was performed. Each artery was scanned three times in succession with an interval of about 5 minutes to allow transfer of the data to the mini computer.

If ultrasound signals originating from different reflectors are used to calculate the attenuation function, correction for the influence of the pressure coupling functions is necessary (see chapter 2 , section 2.4 ). From the results of chapter 4 , however, it appeared that the pressure coupling function can fluctuate drastically with the position and orientation of the tissue interfaces. Especially the position of the vessel in the direction perpendicular to the imaging plane is not known. Therefore, the influence of pressure coupling effects has to be reduced. By using distance pads of different sizes between the transducer and the skin, in all 8 subjects, the anterior wall of the artery could be examined at approximately the same distance from the transducer. Pressure coupling effects are then similar in different subjects and need not to be corrected for, if only data from this series of measurements are compared with each other. By choosing this distance in the far field, it is possible to further reduce the influence of pressure coupling effects.

The pulse protocol used in all examinations is listed in table 7.2. Five different emission frequencies are used and at every frequency 3 pulses with different durations are emitted. The last pulse in the protocol is a conventionall pulse (short, broadband, 1 period at the centre frequency). The echo signals received upon emission of this pulse allow creation of a conventional (broadband) image for comparison with narrowband images. The Pulse Repetition Frequency (PRF) was $8 \mathrm{kHz}$. The use of a 16 pulse protocol implies that for an image consisting of 64 adjacent lines, 1024 lines must be acquired. Acquisition of a complete image then takes $1 / 8$ th of a second.

\subsection{Imaging, attenuation and wall thickness}

\subsubsection{Broadband and narrowband images}

The amplitude signals obtained at each frequency (see equation 7.1) can be displayed on a grey scale monitor, similar to conventional B-mode imaging. To allow comparison of the results of broadband and narrowband signals, no compression or any other image-processing was performed on the raw amplitude data.

To demonstrate the use of the NBMF method for scattered ultrasound, in figure 7.5 a broadband B-mode image (left) and a narrowband image (right) of a tissue mimicking phantom are shown. The bulk material of the tissue mimicking phantom is agar jelly, with a strength of $3.0 \mathrm{~g}$ agar per $100 \mathrm{ml}$ water. In the phantom, 2 cysts ( $8 \mathrm{~mm}$ diameter), containing different concentrations of scattering silicagel particles, are embedded. The centers of the cysts are indicated by the squares with thick white edges. There is no obvious loss in resolution. As was shown in chapter 5, side peaks, resulting from the Wiener-inverse filtering of narrowband pulses, are sufficiently suppressed by correlating the result of two 


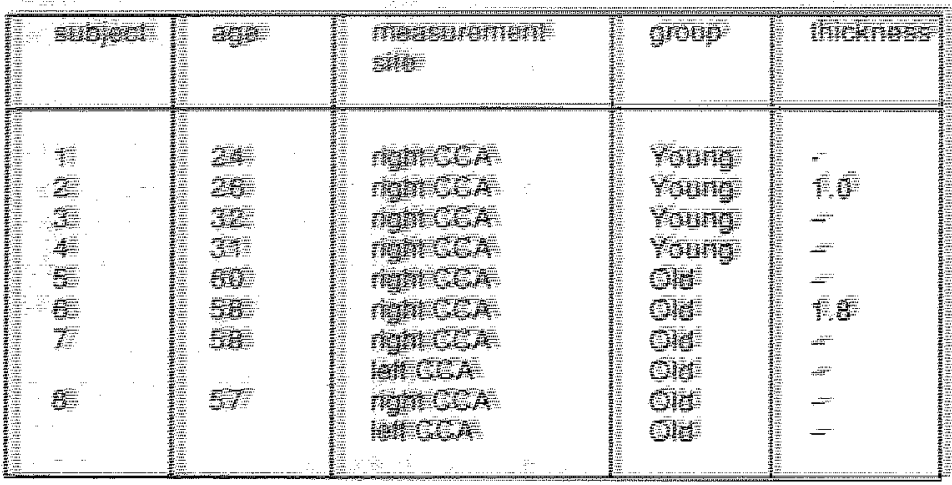

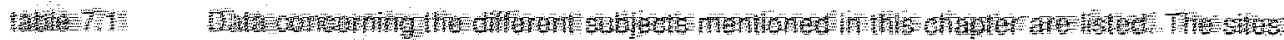
की

\begin{tabular}{|c|c|c|}
\hline 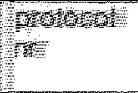 & 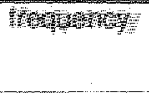 & mombirs \\
\hline 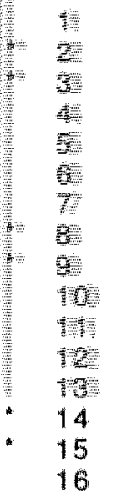 & 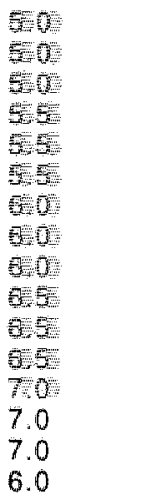 & 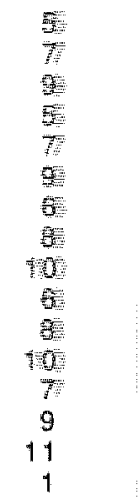 \\
\hline
\end{tabular}

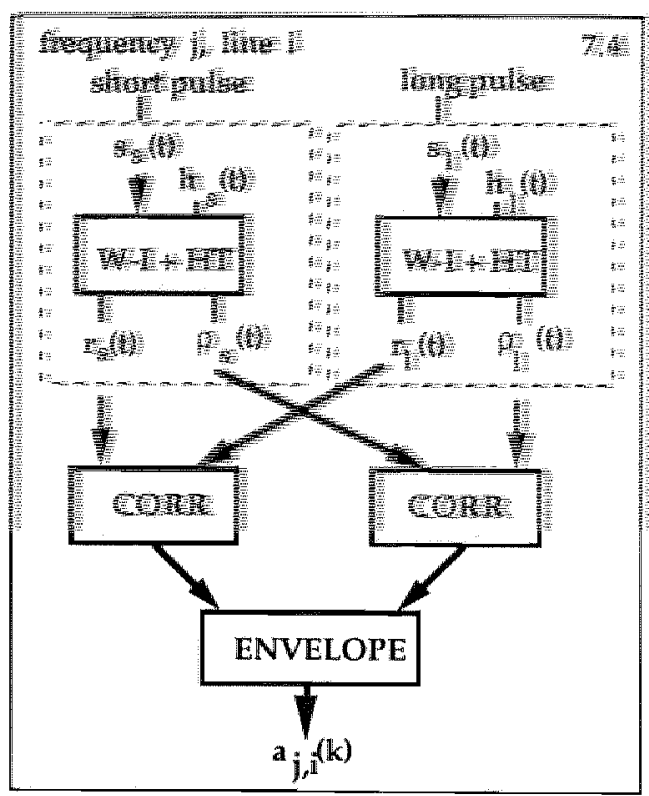

table 7.2 The protocol of pulses, used in the measurements presented in this chapter. The center frequency of each pulse is shown in the second column. The duration of the pulse (expressed as the number of periods of a harmonic signal) is shown in the third column.

figure 7.4 Processing of a pair of narrowband pulses. The duration of the pulses is different, which is indicated by the indices I (long) and $s$ (short). The Hilbert transforms of the Wiener-inverse fillered signals are readily available and they are used to calculate an amplitude (envelope) signal. 

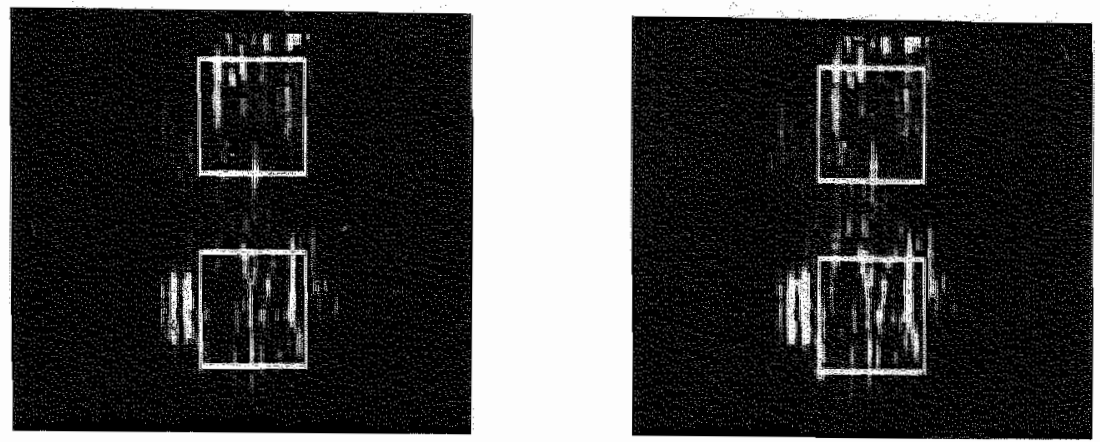

figure 7.5 Conventional broadband image of a tissue mimicking phantom containing two round "lesions", together with a narrowband image of the same phantom. The lesions are the circular regions with bright spots around the two squares.

pulses with different length: there are no additional bright lines (side peaks) in front and after strong reflections.

In figure 7.6 a conventional B-mode image (upper left) and three narrowband images of the common carotid artery of a young volunteer (subject 2), derived from the same measurement, are shown at different frequencies $(5.0,6.0$ and 7.0 $\mathrm{MHz}$. In figure 7.7 the same series of images is shown for one of the patients. The pulses used in the calculation of the images are indicated with asterisks in table 7.1. First of all, it should be noted that, as already demonstrated numerically in chapter 5 , there is no obvious loss of resolution in the narrowband images in comparison with the broadband images. Further, bright regions in the narrowband images correspond to bright regions in the conventional $B$-mode image, which means that no significant artifacts are introduced (edge errors and sidepeaks).

\subsubsection{In-vivo attenuation measurement}

\subsubsection{Estimation of attenuation values}

The frequency dependence of the attenuation caused by the arterial wall can be investigated by comparing the power of the deconvolved narrowband signals in the anterior and posterior wall regions (see figure 7.8). After correlation of the Wiener-inverse filtered signals, the resulting amplitude values are proportional to the square of the reflectivity function. The power $P_{j}$ in a Region Of Interest 

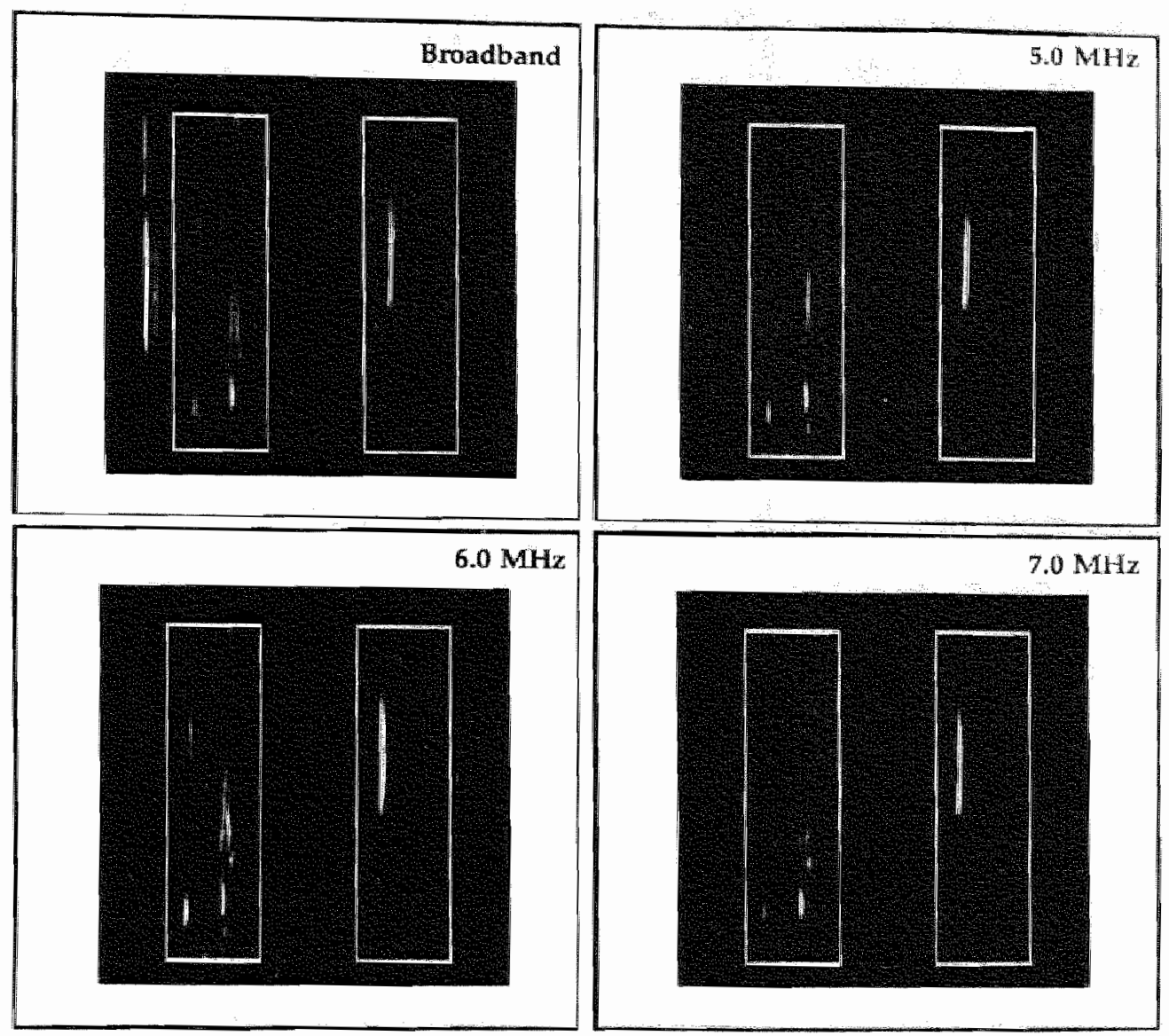

figure 7.7 Conventional broadband image (upper left) of the right common carotid artery of a patient. Three narrowband images, at different center emission trequencies are also shown. The center frequency is indicated in the igure. In horizontal direction, the image corresponds $102.5 \mathrm{~cm}$ allong the direction of the artery. Ir vertical direction, the image corresponds 10 an investigaled depth of $1.9 \mathrm{~cm}$. (for orientation see figure 7.8 ) 
(ROI), at frequency j, can then be estimated simply by calculating the mean of the amplitude values in the ROI:

$$
P_{j}=\frac{1}{\left(m_{2}-m_{1}\right)\left(l_{2}-l_{1}\right)} \sum_{k=m_{1}}^{n_{2}} \sum_{i=l_{1}}^{l_{2}} a_{j, i}(k)
$$

where $l_{1}$ and $l_{2}$ indicate the lower and upper line of the ROI, respectively, and $\mathrm{m}_{1}$ and $\mathrm{m}_{2}$ indicate the begin and end of the $\mathrm{ROI}$ in axial direction, respectively (see figure 7.8). The overall attenuation value [dB scale] at frequency $j$ is then obtained by dividing the average power in the anterior and posterior wall regions:

$$
\left.A_{j}=10^{10} \log \left[P_{i, p} / P_{j, a}\right)\right]
$$

where $\mathbb{P}_{j, p}$ and $P_{j, a}$ are the average power in the posterior and anterior wall regions, respectively. In the approach suggested above, a number of assumptions are made. These assumptions are ellucidated in the appendix.

\subsubsection{Results for in-vivo attenuation measurements}

The procedure described above was applied to the data obtained from the common carotid arteries of the young volunteers and the patients. The regions of interest were selected in the sites of the anterior and posterior walls (see figures 7.6 and 7.7 , rectangles with thick white edges). They were 64 lines long (entire length of the B-mode image) and 256 sample points wide. In the upper panel of figure 7.9, the average of the attenuation (according to equation 7.3) for the two groups is shown at three distinct frequencies. The different groups are denoted as "young" (young volunteers) and "old" (patients measured at a position where there was no evidence for a lesion). The average attenuation is clearly different between the groups, but it should be noted that the variation in the values for each subject can be very large. This can be seen in the bottom panels of figure 7.9, where the attenuation values of the individual measurements are shown in a scatter diagram. Even registrations in one person, performed at approximately the same site, show large variations (see figure 7.10). Two registrations from the group of young volunteers were not included, because the quality of the image was too poor due to mis-alignment of the transducer and the vessel. For the same reason, two scans in the other group were not included.

\subsubsection{Structural information}

In some regions both the narrowband images and the broadband $\mathrm{B}$-mode images show the layered structure of an artery, as can be seen in figures 7.6 and 7.7 . The interfaces between the layers are then visible as bright lines in the image, but generally these lines do not extend over the entire length of the artery. The cause for this phenomenon is that not everywhere along the vessel the tissue interface is perpendicular to the axis of the ultrasound beam. The bright lines can be seen 


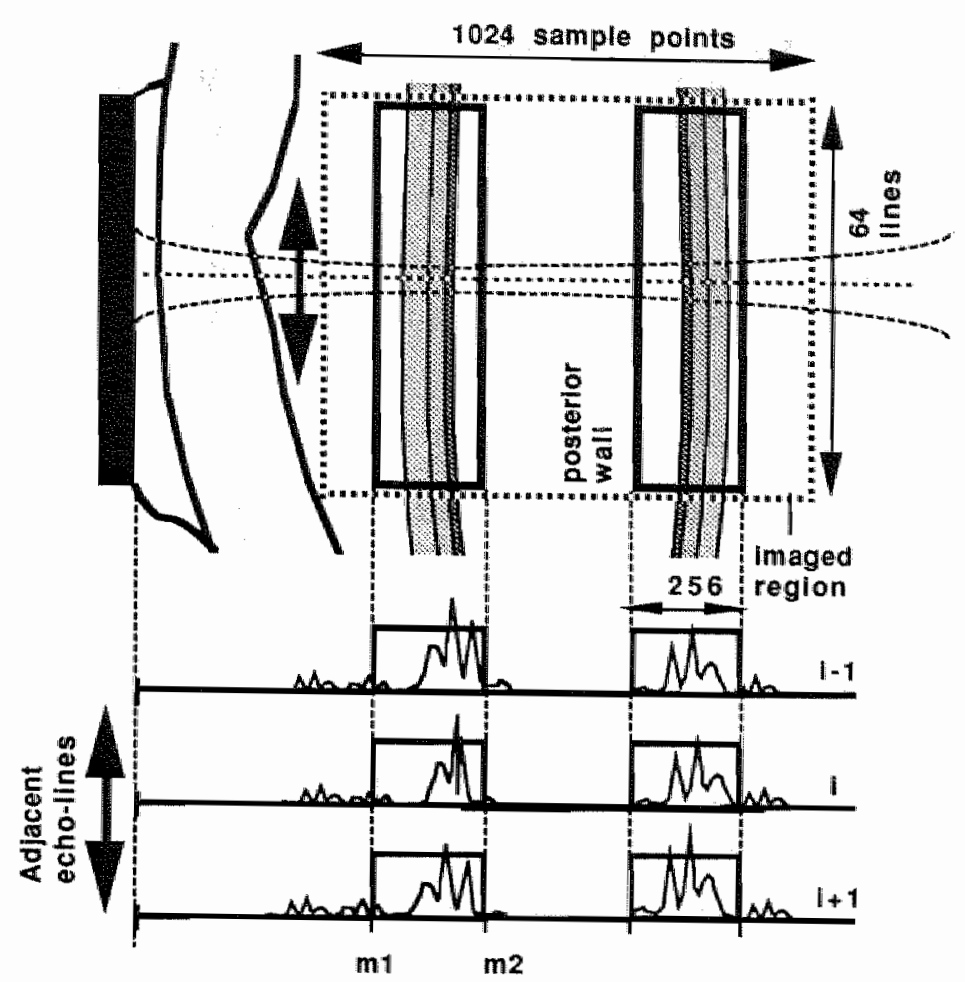

figure 7.8 The imaged region of a common carotid artery is shown. The regions of interest corresponding to the anterior and posterior walls are indicated. The calculation of the average power at one of the frequencies (i) is also shown.

in both broadband and narrowband images, which means that there is no new structural information in the narrowband images.

A common method to measure the thickness of different tissue layers is to place calibration markers directly on the B-mode image. Because the exact position with maximum brightness cannot be determined on a $B$-mode image due to the display resolution, this method is not reliable. Also, there may be variations due to the placement of the markers by different investigators. The thickness of the different layers can therefore not be measured objectively from the B-mode images, although some investigators claim a good accuracy (Pignoli et al 1986, Poli et al 1988).

A more objective method to reveal structure in an image is the calculation of the two dimensional autocorrelation function of the whole image. Because of the fact that the different layers are not visible over the entire length of the artery, this approach is not applicable for ultrasound images with a quality similar to 
that of the images shown in the figures 7.6 and 7.7 . If the artery lies parallel to the Iransducer, it is better to calculate the one dimensional autocorrelation function $\mathrm{C}(\mathrm{t})$ for a number of adjacent echo lines in a Region Of Interest. If the depth of the ROI is not much larger than the thickness of the vessel wall, it is faster to calculate the autocorrelation function via the frequency domain:

$$
C(\tau)=F^{-1}\left(|\mathrm{~A}(\mathrm{O})|^{2}\right)
$$

where $F^{-1}$ denotes the inverse Fourier transform operation and $A(f)$ is the Fourier transform of the amplitude signal of subsequent echo lines (equation 7.1).

The autocorrelation is always maximum at zero time shift. The time shift at which a second peak appears corresponds to the roundtrip distance between the reflectors (constant speed of sound assumed). By averaging the autocorrelation functions for all adjacent echo lines, an estimate of the average thickness of the arterial wall in a region of interest is obtained by determining the position of the second peak in the averaged autocorrelation function. Typical average autocorrelation functions are shown in figure 7.11. In the left panel, the average autocorrelation function of a narrowband image was calculated in a certain region of interest. In the right panel, the same function was calculated using the amplitude signal of the broadband signal. The peaks in the average autocorrelation function calculated from the narrowband signal, can be easier distinguished from their background. This difference between deconvolved narrowband and broadband signals was generally observed. The time shift is transformed to a distance scale, assuming a speed of sound of $1500 \mathrm{~m} \mathrm{~s}^{-1}$.

Besides the functions shown in figure 7.11, which were found for a young volunteer, the same calculation was performed in a good image of one of the patients. Typically, the thickness of the anterior wall, calculated from the time shift at which the side peaks are found (see figure 7.11), was $1.0 \mathrm{~mm}$ in the young volunteer, whereas the thickness of the anterior arterial wall of the patient was $1.8 \mathrm{~mm}$ (speed of sound assumed to be $1500 \mathrm{~m} \mathrm{~s}^{-1}$ ). Unfortunately, the quality of most images obtained from the different subjects was too poor to allow calculation of the autocorrelation function.

\subsection{Discussion}

From the narrowband images obtained from the common carotid arteries of different subjects, it can be concluded that correlation of Wiener-inverse filtered narrowband signals results in amplitude signals with a resolution similar to the resolution obtained using broad band ultrasound pulses. The results from the simulated data used in chapter 5 were thereby confirmed (see also Linssen et al 1991). The use of narrowband images by themselves was not investigated, but Kelly-Fry and colleagues (1988) and Friedman and colleagues (1989) demonstrated the use of narrowband filtered signals for improving the visibility of focal. lesions (tumors). Because the amplitudes of the reflections from vessel wall regions depend on the orientation of the transducer and the wall, the use of 


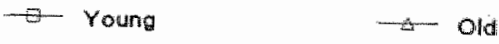
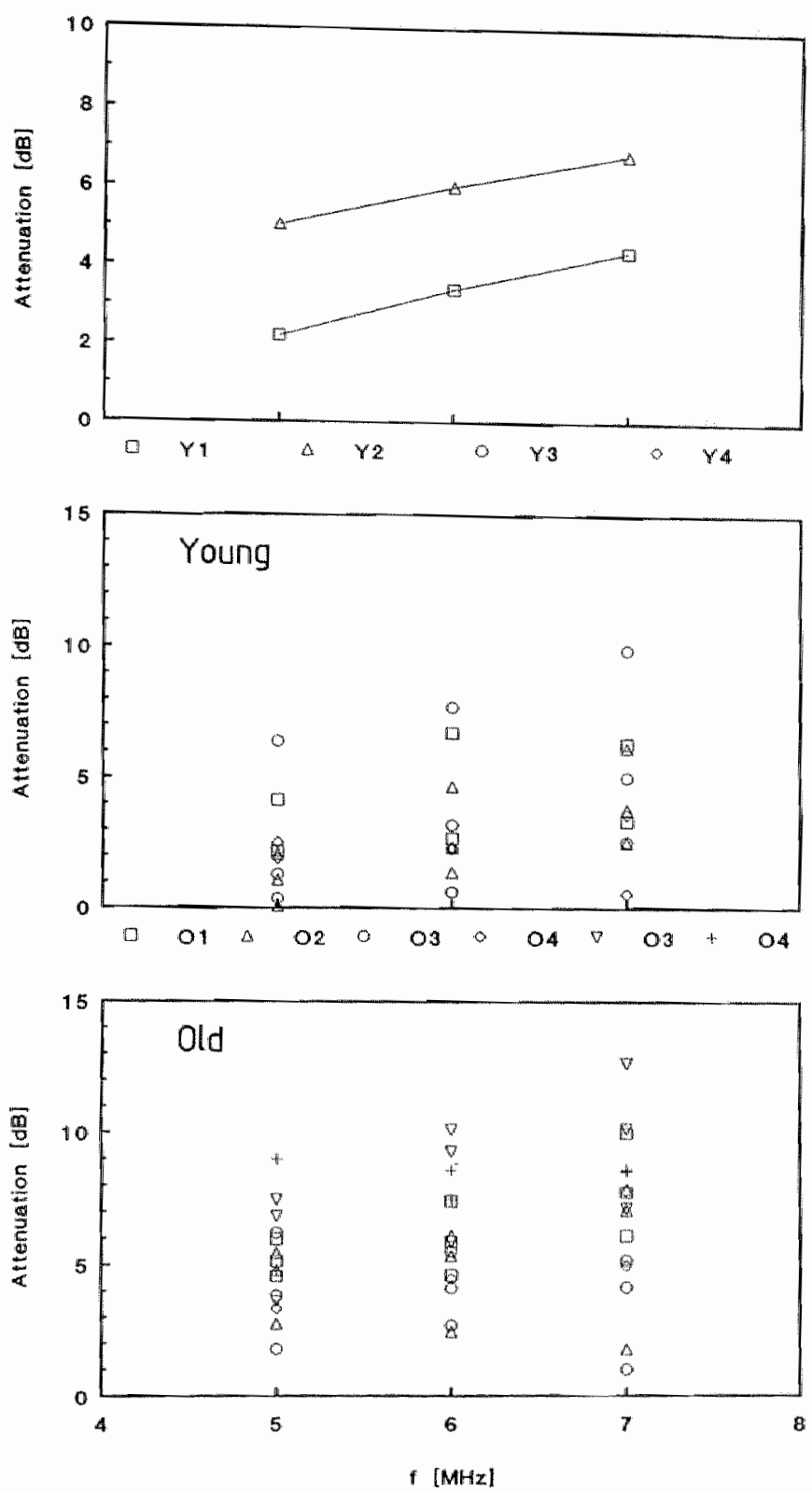

figure 7.9 Top figure: average of the attenuation values (dB-scale) at three different trequencies (horizontal axis) for the group of young and old subjects.

Bottom figures: estimated values for the attenuation for the group of young and old subjects are shown as scatter diagrams. Different subjects are indicated by different symbols. 
$-6-Y_{2}$ acan $1-42 \operatorname{acan} 2-Y_{2} \operatorname{scan} 3$

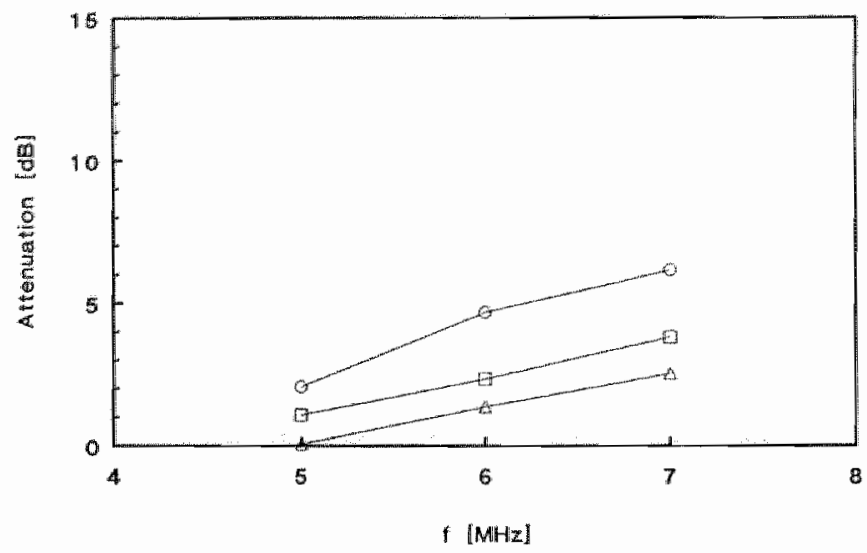

figure 7.10 The attemuation values in the three measurements performed with one of the subjects. The results of each measurement are connected by lines.
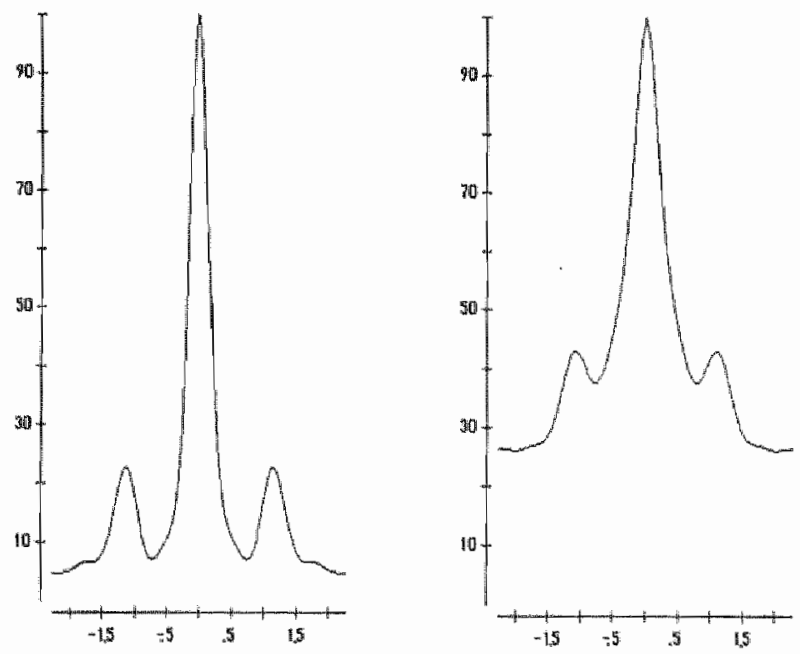

figure 7.11 The averaged autocorrelation function for a region of interest in the narrowband $B$ mode image of a young volunteer is shown on the left. The averaged autocorrelation function for the same region, but calculated with conventional amplitude data, is shown on the right. Values along the horizontal axis are already converted to millimeters (assumed speed of sound $=1500 \mathrm{~ms}^{-1}$ ) Vertical axis scaled to 1.0 at $\tau=0$. 
narrowband images alone may be limited in vascular imaging. The NarrowBand Multi-Frequency method, however, can also be used lo investigate the attenuation (and other frequency dependent acoustic parameters) in large organs (Kuc and Schwarz 1979). Other parameters that can also be assessed from high resolution B-mode images are the backscatter coefficient (Ferdeghini et al 1991), "SNR"-imaging (Verhoeven et al 1991) and parameters like average scatterer dimensions and average scatterer spacing (Insana et al 1990). Its major advantage over the use of broadband ultrasound then is the higher spectral Signal to Noise Ratio of the narrowband pulses. Another application of high-resolution narrowband images at different frequencies is frequency compounding. This method to reduce the speckle pattern has been known for quite some time, but the loss in resolution has always been a problem (Trahey et al 1986, Gatenby et al 1989).

Although the number of observations is too small to allow statistically significant conclusions, the average attenuation values for the two groups of subjects show clear differences. However, there is a large variation in the results obtained in one and the same subject. Although differences in the trequency dependence can be seen in the averaged attenuation curves, further clinical evaluation is needed. The higher attenuation values in the group of patients ("old") may also be the result of a larger thickmess. Because this parameter could be measured in only two subjects, correction for this influence was not possible. For characterization of the tissue type, however, this correction is necessary. The large intra-individual variation is caused by the strong dependence of the amplitude of the reflections on the orientation of the transducer with respect to the vessel wall (pressure coupling effects, Linssen and Hoeks in press). In chapter 4 it was demonstrated that small lateral displacements of a smooth cylindrical surface can already result in large amplitude variations (more than 50\% amplitude reduction for a displacement of $1 \mathrm{~mm}$ ). Locally different tissue properties and the local shape of the reflecting tissue interface may further contribute to these variations. The variation in amplitude was also visible in the broadband images, and from inspection of the actually received signals it appeared that the variations in amplitude between adjacent lines were actually present in the measured signals and that they were not the result of errors in the deconvolution method. Because no correction for pressure coupling effects was performed, it is not allowed to compare the data with in-vitro results from other investigators (Sarnelli et al 1986). Qualitatively, they also found a higher attenuation in walls with increased amount of connective tissue (something which can be expected in older people).

The intra-individual variation in attenuation data can be reduced by averaging a large number of scans $(>64)$ at one measurement site. At this moment acquisition of such a large number of scans is not possible due to the limited storage capacity of the Data Acquisition System (DAS). It is also possible to improve the conventional B-mode image that the ultrasound investigator uses to manipulate the transducer. Now, the amplitude information was logarithmically compressed, so that variations in signal amplitude due to mis-alignment are not immediately visible. Further, real-time edge enhancement would be useful in 
order to manipulate the ultrasound beam through the centre of the artery. A different solution may be the use of a transducer with small dimensions (diameter $<10 \lambda$ ). Such a transducer has a wide beam, so that small deviations in the alignment of the transducer and the vessel do not severely influence the amplitude of the received signal. Also, the transition for near field to far field is at a smaller distance from the transducer than for transducers with large dimensions. Part of the problems, associated with mis-alignment of the transducer and the vessel, however, cannot be solved, because most arteries are slightly curved (e.g: measurement in the carotid bulb will always be troublesome).

The method to assess the thickness of the vessel wall, as described in section 7.3.3, is more objective than the method using calibration markers, positioned by the examiner. But again, the problem of mis-alignment of vessel and transducer causes large problems in visualizing the structure of the arterial wall. Suggestions to improve the handling of the transducer were made above. In situations where the quality of the image is poor, for example, due to reverberations, the method using calibration markers is better, because the examiner can use its anatomical knowledge to discriminate between different bright regions. Also, to date, the capability of the human mind to discern structure in an image is not surpassed by any computer algorithm.

The attenuation values obtained with the NarrowBand Multi-Frequency method may be useful in the characterization of the tissue in the arterial wall. The attenuation values are not specific for the tissue, because the thickness of the vessel wall could not be determined. If problems concerning the orientation the transducer can be reduced by better on-line imaging of the artery, it can be expected that the variation in the attenuation values will decrease. To this end, it is also necessary to average the result of a large number of scans. Until now, it was possible to make only 3 scans per artery. If a larger DAS becomes available, or if the data processing is performed in real-time, it is possible to average a large number of scans ( $>50$ ). Also, it is better to make the acquisition during the diastolic phase of the heart cycle (motion artefacts reduced). The measurements described in this chapter were performed at arbitrary sites, during an arbitrary phase of the heart cycle. Together with information about the thickness of the vessel wall, and information about the mechanical properties of the vessel (Hoeks et al 1990), characterization of the tissue in the arterial wall may be possible.

\section{References}

Ferdeghini E.M. Pinamonti B. Picano E Lattanzi F. Bussano R. and Slavich G (1991): Quantitalive texture analysis in echocardiography: application to the diagnosis of myocarditis. J. Clim. Utrasound 19 $263-270$.

Friedman P.A. Sommer F.G. Chen H. S. Rachlin D.J. Hoppe R. (1989): Characterization of splenic structure in Hodgkin disease by using narrow-band filtration of backscattered ultrasound. As/R 152 $1197-1203$

Gatenby J.C. Hoddinot J.C. and Leeman S. (1989): Phasing out speckle. Phys. Med. Biol. 34 16831689 
Hoeks A.P.G. Brands P.J. Smeets F.A.M. and Reneman R.S. (1990): Assessment of the distensibility ol superficial arteries. Untrasound in Med. \& Biol. 16 1121-1128

Insana M.F. and Hall T.J. (1990): Parametric ultrasound imaging from backscatter coefficient measurements: image formation and interpretation. Uttrasonic Imaging 12245 -

Kelly-Fry E. Morris S.T. Jackson V.P. Holden R.W. and Sanghvi N.T. (1988): Variation of the transducer frequency output and receiver band-pass characteristics for improved detection and image characterization of solid breast masses. Ultrasound in Med. Biol. 14 Sup. $1143-161$

Kuc R. and Schwartz M. (1979): Estimating the acoustic attenuation coefficient slope lor liver from re flected ultrasound signals. IEEE Tr. on Sonics and Uitrasonics SU-26 $353-362$

Linssen F.M.J. and Hoeks A.P.G. (1992): Computation of the signal distortion of reflections from specular reflectors by impulse ray modeling. Accepted for publication in J.Acoustical Soc. Am.

Linssen F.M.J. Hoeks A.P.G. and Brands P.J. (1991): The use of narrowband ultrasound for characterization of lissue in a thin layer. Phys. Med. Biol. $361319-1330$

Pignoli P. Tremoli E. Poli A. Oreste P. and Paoletti R. (1986): Intimal plus medial thickness of the arterial wall: a direct measurement with ultrasound imaging. Circulation $74 \quad 1399-1406$

Poli A. Tremoli E. Colombo A. Sirtori M. and Pignoli P. (1988): Ultrasonographic measurement of the common carotid artery wall thickness in hypercholesterolemic patients. Atherosclerosis 70 253-261

Sarnelli R. Landini L. and Squartini F. (1986): Atherosclerotic detection by ultrasounds. Appl. Path. 4 $270 \cdot 275$

Trahey G.E. Allison J.W. Smith S.W. and von Ramm O.T. (1986): A quantitative approach to speckle reduction via frequency compounding. U/trasonic Imaging $\mathbf{8} \quad 151-164$

Verhoeven J.T.M. Thijssen J.M. and Theeuwes A.G.M (1991): Improvement of lesion detection by echographic image processing: signal-to-noise-ratio imaging. Uitrasonic Imaging 13 238-251 


\section{Appendix}

The simplified model for uitrasound signals, used in chapter 5 and subsequent chapters is:

$$
s_{i}(t)=w_{i}(t) * r_{i}(t)
$$

Of course, any signal can be represented by this model, but then it does not have a physical meaning. From the derivation given is section 2.3 , it is known that this model only is a meaningful representation of the signal received from a number of distinct reflectors if the Pressure Coupling Function (PCF) and the attenuation function of the different reflectors are flat within the frequency bandwid th of the emitted signal $\left(w_{i}(t)\right)$. The attenuation is generally a smooth function of frequency. From the numerical model derived in chapter 4 , it follows that the amplitude spectrum of the PCF is flat and smooth if the reflecting surface is in the far field of the transducer. For sufficiently narrowband signall, the amplitude spectrum of the PCF can also be approximated as a constant if the reflector is in the nearfield of the transducer. The reflectivity function $r(t)$ is then written as:

$$
\begin{aligned}
& r_{i}(t)=\sum_{j=1}^{N} C_{i, j} * \delta\left(t-\tau_{j}\right) \\
& C_{i, j}=C_{i, r, j} C_{i, p, j} C_{i, a_{i} j}
\end{aligned}
$$

where $C_{i, p, j}, C_{i, a, j}$ and $C_{i, r, j}$ are the average values of the $P C F$, the attenuation function and the reflection coefficient for the $j$-th reflector, and for the center frequency of the $i$-th pulse, respectively. Due to the limited bandwidth in which $R_{i}(f)$ can be determined by Wiener-inverse filtering or any other deconvolution method, $r_{i}(t)$ is not a series of $\delta$-functions, but it is the superposition of a number of broader functions $\mathrm{d}\left(\mathrm{t}-\tau_{\mathrm{j}}\right)$ :

$$
r_{i}(t)=\sum_{j=1}^{N} C_{i, j} * d\left(t-\tau_{j}\right)
$$

Denoting the Hilbert transform as $p_{i}(t)$, the envelope of $r_{i}(t)$, Env $\left[r_{i}(t)\right]$, can be calculated from:

$$
\operatorname{Env}\left[r_{i}(t)\right]=\sqrt{r_{i}(t)^{2}+\rho_{i}(t)^{2}}
$$

If the emitted waveform $w_{i}(t)$ is exactly known, the shape of $d(t)$ is shown in figure 5.3. If the peak of $d(t)$ is sufficiently narrow, the peaks in the envelope of $r_{i}(t)$ can be discriminated. The peak amplitudes of the different peaks are proportional to the reflectivity $C_{i, j}$ of the distinct reflectors. 
For a series of identical reflectors $\left(C_{i}, p, j=C_{p}\right)$ with the same reflectivity $\left(C_{i, r_{j},}=\right.$ $C_{\mathrm{r}}$ ), the peak values are proportional to the attenuation term $C_{i, a, j}$. Because $C_{i, a, j}$ represents the average attenuation value within the frequency bandwidth of the $\mathrm{i}$-th pulse, the attenuation due to the tissue between two tissue interfaces at distances $d_{1}$ and $d_{2}$ is (see chapter 2 , equation 2.27):

$$
\gamma\left(f_{i}\right)=\frac{-1}{2\left(d_{2}-d_{1}\right)} \log \left(\frac{\left|C_{i j+1}(f)\right|}{\left|C_{i j}(f)\right|}\right)
$$

where $f_{i}$ is the center frequency of the $i$-th pulse. Evaluation of equation 7.A6 for different center emission frequencies, results in a discrete sampling of $\gamma(f)$.

For narrowband pulses, the amplitude spectrum around the center frequency $\mathrm{f}_{\mathrm{i}}$ is estimated by averaging in a narrow frequency band around $f_{i}$. Mostly, the attenuation coefficient $\gamma(f)$ is expressed in $\mathrm{dB} / \mathrm{cm}$. Equation 2.27 is then expressed as:

$$
\gamma\left(f_{i}\right)=\frac{-1}{2\left(d_{2}-d_{1}\right)} 20^{10} \log \left\{\frac{\left|\bar{A}_{2}(f)\right|}{\left|\bar{A}_{1}(f)\right|} \mid\right.
$$

or, using the power spectrum:

$$
\gamma\left(f_{i}\right)=\frac{-1}{2\left(d_{2}-d_{1}\right)} 10^{10} \log \left\{\frac{\left|\overline{A_{2}(f)}\right|^{2}}{\left|\overline{A_{2}(f)}\right|^{2}}\right\}
$$

where ${ }^{-}$denotes the averaging operation.

The envelope of the signal, processed according to figure 7.4, is proportional to the square of the amplitude of the reflectivity function. According to Parceval's theorem, the power in the frequency domain is equal to the power in the time domain. Therefore, for narrowband ultrasound pulses, the spectral power of the signal in a Region Of Interest (ROI) can be assessed by averaging the amplitude signal (according to equation 7.1) in the ROI.

$$
P_{j}=\frac{1}{\left(m_{2}-m_{1}\right)(12-11)} \sum_{k=m_{1}}^{m_{2}} \sum_{i=11}^{l_{2}} a_{j, i}(k)
$$

where $I_{1}$ and $l_{2}$ indicate the lower and upper line of the ROI, respectively, and $\mathrm{m}_{1}$ and $\mathrm{m}_{2}$ indicate the begin and end of the ROI in axial direction (see figure 7.7). If the average power is determined in two tissue region, at distance $\mathrm{d}_{1}$ and $\mathrm{d}_{2}$, respectively, in equation 7.A8, $\mathrm{P}_{1}$ and $\mathrm{P}_{2}$ can be substituted for the averaged power spectra. 
CHAPTER 8

\section{DISCUSSION, CONCLUSIONS AND RECOMMENDATIONS}


Chapter 8 


\subsection{General discussion and conclusions}

Non-invasive ultrasonic tissue characterization is necessary to detect early atherosclerotic changes in the arterial wall, because other commonly used methods do not give information about the composition of an arterial wall. In this thesis, it was demonstrated that the use of narrowband ultrasound pulses with different center emission frequencies (NarrowBand Multi-Frequency method) results in frequency specific information which is more reliable than the spectral information obtained using broadband ultrasound pulses. Using numerical models for the sound field of ultrasound transducers, it was demonstrated that the position of a reflecting tissue interface (e.g. layers in an arterial wall) greatly affects the received ultrasound signal. The sound field calculations were based on realistic transducer parameters. A method to assess these parameters was developed.

\subsubsection{Discussion on NarrowBand Multi-Frequency method}

If one wants to characterize the structure and acoustical properties of tissues in a thin layer, like an arterial. wall $(0.1-2.0 \mathrm{~mm})$, a major problem is to discern the different tissue layers. If only the envelope of the received signals is used for creating the image, resolution increases with decreasing pulse duration. Hence, for imaging thin layered structures, the use of high-frequency, broadband ultrasound systems seems obvious. For tissue characterization, however, short pulses suffer from a poor spectral Signal to Noise Ratio (SNR). Also, frequency dependent attenuation tends to reduce the available bandwidth. Therefore, the use of narrowband ultrasound at different center emission frequencies is suggested in this thesis.

The use of narrowband filters to investigate the reflected ultrasound signals in a narrow frequency band is not new. Friedman and colleagues (1989) demonstrated the use of narrowband filtered signals to improve visibility of regions with deviating backscattering properties. Kelly-Fry and colleagues (1988) suggested the use of a very broadband transducer and emission of broadband pulses with variable center emission frequency. Subsequent narrowband filtration then resulted in frequency specific images. The problems associated with the poor spectral SNR, however, are not solved by the use of narrowband filters. Emission of narrowband pulses at different frequencies has been suggested as a method to reduce the speckle pattern normally observed in B-mode images (frequency-compounding, Gehlbach and Sommer 1987, Trahey et al 1986), but the loss in axial resolution, due to the long duration of narrowband pulses, was considered as a major disadvantage of frequency compounding.

Because of the use of long (narrowband) excitation pulses, restoration of the axial. resolution is also the major problem in the NarrowBand Multi-Frequency method. Deconvolution of the amplitude information, available in the B-mode image was not very successful, because interference effects cannot be taken into 
account (Jeurens et al 1986, Loupas ef al 1989). The use of the Radio-Frequency (RF) signal was suggested by Herment et al (1989a and $1989 \mathrm{~b}$ ). The problem with broadband ultrasound pulses, however, is that the waveform changes while traveling through the insonified tissue. Therefore, the success of deconvolving broadband ultrasound is also very limited. In seismological literature, several alternative deconvolution methods can be found (Robinson and Treitel 1980). Some examples are $1_{1}$-deconvolution (Levy and Fullager 1981) and fast Kalman filtering (Crump 1974). These methods, however, are based on the assumption that the signal originates from a small number of reflectors. Because the number of scatterers in ultrasound signals is large, application of these methods in the analysis of ultrasound signals is limited.

A great advantage of monochromatic pulses with a long duration is that only the last and first part of the reflected pulse are prone to distortion (RCF). Because of this feature and the excellent spectral SNR it is possible to use a Wiener-inverse filter to restore axial resolution (chapter 5). Wiener-inverse filtering, however, results in undesirable artefacts due to the narrowband character of the emitted pulses. Correlation of the deconvolved result with the Wiener-inverse filtered result of a second signal is necessary to suppress undesirable artefacts (Linssen et al 1991). This second pulse is a pulse with the same frequency but with a longer or shorter duration. "The resulting axial resolution is then similar to the axial resolution which can be obtained using an ideal broadband excitation pulse. The optimal combination of the duration of the pulses was not investigated. Application of this method in practice involves the use of complex ultrasound emitters and dedicated signal processing hardware. Using modern FFTprocessors it must be possible to produce on-line narrowband B-mode images.

Problems can be expected from the limitations on the acoustical power allowed. Most limitations are expressed as the maximum power per unit of area. The energy per pulse increases drastically when narrowband pulses are used (one of the reasons for the good results). Moreover, the pulses must be emitted at a very high PRF to obtain real-time information. This means that it is very well possible that current safety standards are violated. The concentration of the signal power within a narrow frequency band, however, allows a decrease in signal amplitude. Also, the peak amplitudes of the emitted pulses are much lower than the amplitude of broadband pulses with a similar SNR.

Due to the non-zero duration of the reflected signals, it is possible that reflections are cut off at the beginning and end of the received echo signal. Wiener-inverse filtering then results in undesirable artefacts. This problem is well known in the field of image processing (correction of motion-blurred images, Lim et al 1991). In vascular imaging, selection of time windows, with their begin and end points in regions with low amplitude signals, is possible and a good solution to avoid edge truncation errors. For general application of the NBMF method, however, this may be a problem. In that case, a way to reduce edge errors is the use of a (Tukey) smoothing window.

In chapter 5, the waveform of the electrical input was used as an approximation of the actually received waveform of a single reflector. It appeared that for 
narrowband signals this approximation results in a good restoration of the axial resolution. However, the validity of the approximation becomes poorer if the bandwidth of the ultrasound system is not broad enough. Knowledge of the systems transfer function can be used to improve the deconvolution signal. In chapter 6 it was demonstrated that use of this knowledge results in improved performance of the Wiener-inverse filter for simulated signals (chapter 6). For real ultrasound signals, however, the improvements were less pronounced, probably because the measurement system performs too well. The noise cut off level can then be chosen so low, that the result of the Wiener-inverse filter approximates the systems impulse response and little improvement is possible. Because the side peaks were slightly lower when using corrected deconvolution signals rather than uncorrected signals, impulse response correction was applied in obtaining the results in this thesis. Cepstral filtering is a form of homomorphic filtering which was used in the appendix of chapter 6 to estimate the transfer function of the Color Flow Mapper system used in the measurements in this thesis. This technique is well known in image processing (Gonzales 1987, Oppenheim and Schafer 1975). In the cepstrum domain the transfer function to be estimated must be clearly separated from the noise and electrical input signals. If this is not true, the method will not give the desired result. Using narrowband signals, this means that the separation in center frequencies must be sufficiently small and that the duration of the pulses must be sufficiently long to result in a high-quefrent cepstrum.

In chapter 7 the results obtained in scanning the right common carotid arteries of 4 young volunteers and 4 patients from the neurological clinic are presented. The patients suffered from neurological deficits, associated to atherosclerotic lesions in the carotid artery bifurcation, generally in the carotid artery bulb. After appropriate processing, narrowband images have the same resolution as conventional B-mode images. Values for the overall attenuation caused by the arterial wall can be derived from the amplitude data of the narrowband data obtained at different frequencies. The average values for the attenuation in the right common carotid artery at different frequencies were clearly different for the young volunteers and the patients. The intra-individual variance of the attenuation values, however, was quite large. Again, the problem is the orientation and position of the vessel in the sound field of the transducer (pressure coupling effects). Some suggestions to reduce this influence will be made in section 8.2. The data were obtained using distance pads, to assure that the anterior wall of the vessel was at the same distance for every subject. Correction for differences in pressure coupling effects was therefore not necessary. Because the thickness of the arterial walls could not be determined in all subjects, it was not possible to discern the influence of tissue composition and differences in arterial wall thickness between the groups.

The high Signal to Noise Ratio of narrowband signals is expected to be a major advantage of the NarrowBand Multi-Frequency for tissue characterization and visibility enhancement in large organs and for vascular structures. Moreover, if the voltage of the electrical excitation signal is adapted to the system transfer function at the center frequency, the Signal to Noise Ratio at the different center emission can be constant. This is not possible using broadband excitation signals. 
Also, for narrowband signals, diffraction correction can be easily achieved. Instead of range-dependent diffraction correction spectra (Laugier et al 1987, Verhoef et al 1985), diffraction correction may be achieved by time gain compensation.

\section{1:2 Discussion on methods to assess radiation coupling effects and transducer parameters}

Several characteristics of the ultrasound signals received from the body depend on the uitrasound equipment used. Because it is not practical to measure the soundfield for every waveform and transducer one wants to employ, part of the analysis of ultrasound signals in this thesis is performed using computer simulations. For the calculation of the sound field, many investigators assume that the amplitude of the excitation at the transducer surface is uniform (Stepanishen 1971, Penttinen and Luukkala 1976). In reality, this will be rarely the case. In order to perform realistic simulations, a method to determine the Surface Velocity Amplitude Distribution (SVAD) is needed. Although many sound field models available from the literature, provide the possibility of incorporating the effects of a non-uniform SVAD (Verhoef et al 1984, Ocheltree and Frizzell 1989), the SVAD is still assumed to be uniform, or more of less realistic assumptions are made regarding the non-uniformity. The method described in chapter 3 allows determination of the SVAD from simple pressure amplitude measurements performed in a water tank. The estimation procedure is a Kalman filter (Brammer and Siffling 1975, Jazwinsky 1970). The calculation of the sound field is independent of the Kalman filter. Therefore, transducer geometries, other than circular, can be easily implemented in the estimation program and any method for the sound field calculation (analytical or numerical approximation) can be used. The major restriction of the method proposed is that it must be possible to model the SVAD using only a few unknown parameters. For complex transducer geometries there is the possibility that the SVAD cannot be modeled by a simple parametric function. In that case the method will fail. Most commonly used ultrasound transducers, however, have simple geometries (circular, square, annular array and linear array). A complex SVAD must be determined with the aid of the backprojection method (Schafer and Lewin 1989, Waag et al 1985, Stepanishen and Benjamin 1982). This method is very sensitive to noise in the measured data. The method proposed in chapter 3 is therefore a good alternative for determining the parameters of commonly used transducers.

In chapter 4 an impulse ray model is used to determine the function from which the distortion of a pulse upon reflection at a smooth surface can be calculated: the Radiation Coupling Function (RCF). The proposed algorithm is a modification of the method proposed by Cassereau and Guyomar 1988. The method proposed here uses another method to distribute the rays, originating from a small part of the emitting transducer, into the medium in front of it. The result is, that depending on the configuration of the reflector and the transducer, large savings in computation time can be achieved. This advantage partly vanishes if one wants to investigate transmission through an interface (multiple reflection). From the simulations it follows that minor changes in the position of the 
reflecting surface result in large fuctuations in signal amplitude. Apart from the influence of the position of the reflector in the sound field, there is additional distortion due to the fact that the interface between two tissue layers is not smooth, and further, there is not a sudden jump in acoustical impedance (Chivers and Santosa 1986). Also, an artery is not a straight and round cylindrical tube. The results from the numerical simulations in chapter 4 are therefore only approximations of the real situation. The results, however, can be used to avoid situations in which small variations in the position of the reflector can be expected to have a great influence on the amplitude of the reflections.

\subsubsection{Conclusions}

Below, the conclusions from the thesis are presented.

- Assessment of the actual transducer parameters, in particular the Surface Velocity Amplitude Distribution, can be achieved using the parameter estimation method presented in chapter 3. The agreement between the measured pressure amplitude distribution and the amplitude distribution calculated with the sound field model is a validation of the sound field models in which the Surface Velocity Amplitude Distribution is the same for all frequencies.

- From the results of the impulse ray model presented in chapter 4 it can be concluded that the influence of the orientation of the transducer with respect to the vessel is less critical if the vessel is in the far field of the transducer. For superficial arteries this implies the use of distance pads or transducers with small dimensions.

- The NarrowBand Multi-Frequency method results in frequency specific information, with a good restoration of the resolution and a better Signal to Noise Ratio than results from analysis of broadband ultrasound signals (chapters 5, and 7).

- A major problem in the assessment of tissue parameters of a thin-layered structure, like an arterial wall, is the influence of the orientation of the transducer with respect to the reflecting tissue interfaces (chapter 7 ).

- A high quality imaging system (broadband transducer), together with appropriate image and signal processing is necessary to reduce the variability of the measurements (chapter 7). Also, the influence of the system transfer function on the deconvolution process is less for broadband systems (chapter 6 )

- A high quality imaging system is also necessary to assess the thickness of the arterial wall. Without this information the observed attenuation is not purely a tissue characterizing parameter (chapter 7). 


\subsection{Recommendations}

\subsubsection{Transducers and ultrasound equipment}

Broadband transducers (ratio of bandwidth to center frequency $>1$ ) should be used to allow assessment of the response of the investigated tissues in a frequency range which is as wide as possible. Further, the acousto-electrical sensitivity must be as high as possible because the deconvolution process is susceptible to noise. These recommendations are in agreement with the demands of conventional ultrasound equipment and it can be expected that the performance of ultrasound transducers will further improve in the near future. Especially the development of new piezo-electric materials may contribute to this improvement.

The center frequency of the transducer must be as high as possible because the layers in an arterial wall are very thin (especially at the early stages of atherosclerosis or in normal persons). The bandwidth of a transducer, which determines the resolution that can be obtained, is generally proportional to the center frequency. As attenuation increases with frequency, however, there is a limit to frequencies which can be used for non-invasive ultrasound scanning. For superficial arteries, $10-12 \mathrm{MHz}$ may be the limit. For intravascular ultrasound, much higher frequencies can be used: $20-70 \mathrm{MHz}$.

Improvement of the $B$-mode image that the examiner uses to manipulate the ultrasound transducer is essential, because mis-alignment of the transducer and the artery is a major cause of the large intra-individual variations observed in the estimation of the overall attenuation. Compression of the amplitude data must be omitted, because compression masks the amplitude variations due to mis-alignment. The use of edge enhancement techniques may further improve the B-mode image. Real-time implementation of the NarrowBand MultiFrequency method (display of narrowband B-mode images) may further improve the results.

The use of diffraction-free transducers as recently introduced by Greenleaf and coworkers is highly recommendable ( $L u$ and Greenleaf 1990). Diffraction and radiation coupling effects are disastrous for any kind of tissue characterization, because correction is very difficult, if not impossible, Another possibility for reducing diffraction and radiation coupling effects, is the use of small transducers with a broad beam.

\subsubsection{Measurement procedures}

In order to circumvent the influence of radiation coupling effects on the results from in vivo measurements, the artery must always be in the same region of the transducer. Because of the inter-individual differences in anatomy, the use of distance pads is required. 
Great care has to be taken in orienting the transducer with respect to the vessel, even if distance pads are used to ensure measurements in the far field of the transducer. For a linear array transducer, as used in this thesis, the axis of the vessel must lie parallel to the transducer. Further, the back wall echoes must be clearly visible (preferably showing a layered appearance).

Due to compression and subsequent decompression the thickness of the arterial. wall is not constant during the cardiac cycle. Therefore, subsequent acquisitions of data should be performed during the same phase of the cardiac cycle. This can be achieved using a ECG (Electro Cardio Gram) trigger. To avoid motion artifacts, this should preferable be during the diastolic phase.

\subsection{Prospects of the NarrowBand Multi-Frequency method}

The Wiener-inverse filtering and correlation of the pair of signals received at every distinct frequency must be performed on-line to allow real time imaging. Using a number of modern FFT-processors in parallel, this must be possible.

To a physician it may not be important whether a parameter, derived from the received ultrasound signals, has a physical meaning. If a parameter can be used to discriminate between different tissues and pathological states of a tissue it is possible to visualize abnormalities in the body. In fact, the possibility of cheap real-time visualization is the major reason why ultrasound has become one of the most common imaging modalities. Indeed, parametric imaging has become a topic in recent years. Insana (Insama et al 1990) tried to estimate some parameters related to the spatial distribution and size of the scatterers within a tissue mimicking phantom. Verhoeven and colleagues (1991) showed that visualization of the local SNR of the video image can visualize tissue regions which are not visible in conventional B-mode images. Friedman et al (1989) demonstrated that the reflectivity of a tissue region depends on frequency, and suggested the use of narrowband filtered broadband pulses to visualize certain tissue regions. The high-resolution and frequency specific information available from the NarrowBand Multi-Frequency method offers many new possibilities. Combination of images obtained at different frequencies can result in (parametric) images which enhance the detectability of anomalities in arterial walls, but also in other parts of the body (large organs like liver and spleen).

If real-time imaging is possible, acquisition of data becomes much easier. Then it will also be possible to make a large number of scans of the artery at one site. The intra-individual variation of the attenuation results will then decrease by averaging the results of a large number of scans. Combined measurement of mechanical behavior (Vessel Wall Movement detection), arterial wall thickness (see chapter 8) and overall attenuation (see chapter 8) is necessary to obtain more insight into the development of atherosclerosis, and may eventually result in improved (earlier) diagnosis of minor (pre-)atherosclerotic lesions. 


\section{References}

Brammer K. and Siffling G. (1975): Kalman Bucy Filter; Deterministische Beobachtung und stochastische Filterung. (R Oldenbourg, Munchen and Wien) (in German).

Cassereau D. and Guyomar D. (1988): Computation of the impulse diffraction of any obstacle by impulse ray modelling - Prediction of the signal distortion. J. Acoust. Soc. Amer. $841504-1516$

Chivers R.C. and Santosa F. (1986): Numerical considerations for interface reflections in medical ultrasound. Phys Med. Biol 31 819-837

Crump N.D. (1974): A Kalman fitter approach to the deconvolution of seismic signals. Geophysics 39

Fridedman P.A. Sommer F.G. Chen H. S. Rachlin D.J. Hoppe R. (1989): Characterization of splenic sinucture in Hodgkin disease by using narrow-band tiltration of backscattered ulltrasound. AJR 152 $1197-1203$

Gehlbach S.M. and Sommer F.G. (1987): Frequency diversity speckle processing. Uitrasonic /maging $992-105$.

Gonzales R.C. and Wintz P (1987): Digital image processing. (Addison-Wesley Publishing Company. Reading, Massachusets)

Herment A. Gugllelmi J.P. Peronneau P. and Dumee Ph. (1989a): High-resolution, reflection mode tomographic imaging Part I: principles and methods. Uitrasonic Imaging 11 1-21

Herment A. Guglielmi J.P. Peronneau P. and Dumee Ph. (1989b): High-resolution, reflection mode lomographic imaging Part II: application to echography. Utrasonic maging $11 \quad 22-41$

Insana M.F. and Hall T.J. (1990): Parametric ultrasound imaging from backscalter coefficient measurements: image formation and interpretation. Ultrasonic /maging 12245

Jazwinsky A.H. (1970): Stochastic processes and filtering theory. (Academic Press, New York),

Jeurens T.J.M. Somer J.C. Smeets F.A.M. and Hoeks A.P.G. (1986): The practical significance of two dimensional deconvolution in echography. Utrasonic Imaging 9 106-116

Kelly-Fry E. Morris S.T. Jackson V.P. Holden R.W. and Sanghvi N.T. (1988): Variation of the transducer frequency outpult and receiver band-pass characteristics for improved detection and image charactenization of solid breast masses. Uhrasound in Med. \& Biol. 14 Sup. 1 143-161

Laugier P. Berger G. Fink M. and Perrin J. (1987): Diffraction correction for focused transducers in attenuation measurement in vivo. Ulirasonic Imaging 9248.259

Levy S. and Fullager P.K. (1981): Reconstruction of a sparse spike train from a portion of its spectrum and application to high-resolution deconvolution. Geophysics 46 1235-1243

Lim H. Tan K. C. and Tan B.T.G. (1991): Edge errors in inverse and Wiener filter restorations of molion-blurred images and their windowing treatment. CVGIP: Graphical models and image processing $53 \quad 186-195$

Linssen F.M.J. Hoeks A.P.G. and Brands P.J. (1991): The use of narrowband ultrasound for characterization of tissue in a thin layer. Phys. Med. Biol. 36 1319-1330

Loupas T. Pye S.D. and McDicken W.N. (1989): Deconvolution in medical ultrasonics: practical considerations. Ptys. Med. Biol. 34 1691-1700

Lu J.Y. and Greenleal J.F. (1990): Ultrasonic non-diffracting transducer for medical imaging. IEEE Trans. Uitrason. Ferroelec. Freq. Contr. UFFC-37 438-447 
Merode van T. Hick P.J.J. Hoeks A.P.G. and Reneman A.S. (1988): The diagnosis of minor to moderate atherosclerotic lesions in the carotid antery bifurcation by means of spectral broadening combined with the direct detection of flow disturbances using a multi-gate pulsed Doppler system. Utrasound in Med. \& Biol. $14 \quad 459-464$

Ocheltree K.B. and Frizzell L.A. (1989): Saund field calculations for rectangular sources. IEEE Trans. Utrason. Ferroelec. Freq. Contr. UFFC-36 242-248

O'Donnel M. (1983): Effects of diffraction on measurement on the firequency-dependent ultrasonic attenuation. IEEE Trans. Biomed. Engin. BME-30 $320-325$

Penttinen A. and Luukkala M. (1976): The impulse response and pressure nearfield of a curved ultrasonic radiator. J. Phys. D. 9 1547-1557

Schafer M.E. and Lewin P.A. (1989): Transducer characterization using the angular spectrum method. J. Acoust. Soc. Amer. 85 2202-2214

Stepanishen P.R. (1971): Transient radiation from piston in an intinite planar baffle. J. Acoust. Soc. Amer. 49 270-275

Stepanishen P.R. and Benjamin K.C. (1982): Forward and backward projection of acoustic fields using FFT methods. d. Acoust. Soc. Amer. 71 803-812

Trahey G.E. Allison J.W. Smith S.W. and Von Ramm O.T. (1986): A quantitative approach to speckle reduction via frequency compounding. Uttrasonic /maging 8 151-164

Verhoef W.A. Cloostermans M.J.T.M. and Thijssen J.M. (1985): Diffraction and dispersion effects on the estimation of ultrasound attenuation and velocity in biological tissues. IEEE Trans. Biomed. Engin. BME-32 521-529

Verhoef W.A. Cloostermans M.J.T.M. and Thijssen J.M. (1984): The impulse response af a focused source with an arbitrary axisymmetric surface velocity distribution. J. Acoust. Soc. Amer. 75 17161721

Verhoeven J.T.M. Thijssen J.M. and Theeuwes A.G.M (1991): Improvement of lesion detection by echographic image processing: signal-to-noise-ratio imaging. Ultrasonic Imaging 13 238-251

Waag R.C. Campbell J.A. Ridder J. and Mesdag P.R. (1985): Cross-sectorial measurements and extrapalations of ultrasonic fiellds. IEEE Trans. Sonics Ultrasonics SU-32 26-35

Oppenheim A. and Schater R. (1975): Digital signal processing. (Prentice-Hall, Englewood Clifts)

Robinson E.A. and Treitel S. (1980): Geophysicsl signal analysis. (Prentice-Hall, Englewood Cliffs) 


\section{Summary}

Atherosclerosis is a disease that initially affects only the structures and composition of the tissues within the vessel wall. The local blood low distribution is not notably disturbed by these changes in the vessel wall. Also, minor changes in the composition do not necessarily result in changes of the mechanical properties of the vessel. Therefore, evaluation of blood flow patterns or measurement of vessel wall motion do not give specific information about early stages of atherosclerosis (chapter 1).

Because it is possible to distinguish different types of tissue because of their acoustical properties, determination of the acoustical properties of the tissue within the vessel wall may give more and direct information about the early stages of atherosclerosis. There are several methods available from literature for tissue characterization (chapter 2). A problem generally observed in making longitudinal B-mode images of arteries is that the image depends on the orientation of the transducer. It is difficult to obtain an image in which the different layers of the vessel are clearly visible, both in the region of the anterior and the back wall. Even if different layers are visible, it is not along the entire length of the artery. This problem is caused by the interaction of the ultrasound beam and the (cylindrical) shape of the interfaces between the different tissue layers in the vessel wall as was investigated in chapters 3 and 4 .

In order to make realistic simulations of the sound field of a transiducer, it was necessary to develop a method to determine the parameters of a transducer from sound field measurements. In chapter 3 a method is presented by which it is possible to derive the Surface Velocity Amplitude Distribution (SVAD) on the surface of a circular focussed transducer. The SVAD and other parameters are determined from simple sound pressure measurements. A Kalman filter is used as parameter estimation algorithm because it allow's easy control of the estimation procedure.

The sound field and the geometry and position of a reflecting surface determine the deformation of an acoustic pulse upon reflection by that surface. An impulse ray model is used to calculate the impulse response or Radiation Coupling Function (RCF) of a reflector in the acoustical field of an ultrasonic transducer (chapter 4). It appeared that the RCF of a cylindrical reflector (vessel wall) in the near field of a transducer highly depends on the radius and position of the reflector. The result is that a broadband pulse is severely distorted after reflection at a tissue interface. Because in practice the geometry of reflecting structures is not known, this distortion can not be corrected, but it is found that the deformations are less severe if the reflecting surface is in the far field of the transducer.

Because broadband signals suffer from signal distortions and a poor spectral Signal to Noise Ratio (SNR), a new method for investigating the frequency dependence of the acoustic properties of a (thin-layered) tissue is proposed in chapter 5. By emitting narrowband pulses with different center emission frequencies at a high Pulse Repetition Frequency (PRF), it is possible to assess the frequency 
dependence of acoustic parameters of a tissue. This NarrowBand MultiFrequency (NBMF) method involves ordinary Wiener-inverse filtering of the individual RF-lines to restore the loss in resolution due to the long duration of narrowband signals. Because of the narrowband character of the pulses, however, artefacts arise in the Wiener-inverse filtered signals. By correlating the deconvolution result of a narrowband RF-line with the deconvolution result from a RFline received upon emission of a longer pulse at the same frequency, these artefacts are suppressed. The resolution of the resulting signals is within the limits given by the systems impulse response and therefore similar to the resolution obtained using broadband ultrasound signals.

The quantitative accuracy of the Wiener-inverse filter method is limited if the electrical excitation waveforms, which are used as deconvolution signals, are distorted due to a relatively narrowband system transfer function. Variations in peak amplitude values up to $10 \%$ are found even in computer simulated signals. Therefore it was investigated whether the use of knowledge about the system transfer function can improve the accuracy and resolution (chapter 6). It appeared that the use of spectral amplitude information results in a more uniform resolution for a wide range of center frequencies. If the phase of the transfer function is a smooth function of frequency (for realistic systems almost always true), knowledge of the phase does not improve the resolution (measured as the width of the main peaks at half maximum), but only influences the position of the peaks.

Finally, in chapter 7 the NBMF method is used to assess data from the common carotid arteries of young volunteers and patients from the neurological clinic. Although the method results in frequency specific B-mode images, the problems with the visibility of the different layers are the same as in conventional images. By comparing the average value of the envelope of the deconvolved signals in two regions an estimate of the overall attenuation at a specific frequency between two tissue regions is obtained. Repeating this procedure for different frequencies results in the assessment of the frequency dependence of the attenuation. Clear differences in overall attenuation were found if the results for the different groups are averaged. However, there is a large intra-individual variance in the results, which is probably caused by mis-alignment of the transducer and the artery.

The intra-individual variation may be reduced if the data processing can be performed in real-time. Also, the visualization of the artery on the conventional Bmode image must be improved. It is then possible to reduce the mis-alignment of the artery and the transducer. Acquisition of data synchronous with the cardiac cycle can further reduce the variations in the results. Combination of attenuation values with measurement of the thickness of the vessel wall and assessment of the mechanical behaviour of the vessel can then result in characterization of vessel wall tissue. This will facilitate investigation of the early stages of atherosclerosis and eventually in improved diagnosis. The use of either method alone can not result in the desired information about the composition of the arterial wall. 


\section{SAMENVATTING}

Zowel de bloedstroming als ook het mechanisch gedrag wan het bloedvat kunnen worden bestudeerd gebruikmakend van ultrageluid. Ultrageluid is niet belastend voor de patient omdat er alleen maar een ultrageluidstransducent op de huid moet worden geplatst. Verder worden er alleen onschadelijke geluidsgolven uitgezonden (geen rontgenstraling etc). Ultrageluid wordt veel gebruikt om vernauwingen $\mathrm{tg}, \mathrm{v}$ : atherosclerose (in de volksmond aderverkalking genoemd) op te sporen door middel van detectie van verstoringen van het bloedstroom profiel. Meer recentelijk is het ook mogelijk geworden om de beweging van de bloedvatwanden ten gevolge van de pulserende bloeddruk waar te nemen. Echter, de eerste stadia van atherosclerose worden voornamelijk gekenmerkt door veranderingen in de samenstelling van de bloedvatwand. Plaatselijke lesies (atherosclerolische afwijkingen van de wand) die een gedeelte van het bloedvat afsluiten zijn op dat moment nog niet tot ontwikkeling gekomen en daarom zijn er nog geen eenduidige verstoringen van het bloedstroom profiel warneembaar. Ook de elasticiteit van de wand, die kan worden bepaald door meting van de diameterverandering als functie van de pulserende bloed-druk, geeft geen directe aanwijzingen voor de aanwezigheid van veranderingen in de samenstelling van de bloedvatwand. Vandaar ook dat er in dit proefschrift getracht is een methode te ontwikkelen om d.m.v. analyse van de ontvangen ul-trageluidspulsen meer te weten te komen over veranderingen in samenstelling van de bloedvatwand: weefselkarakterizering m.b.v. ultrageluid (hoofdstuk 1). Een bloedvat dat heel goed kan worden onderzocht omdat het dicht onder de huid ligt, is de gemeenschappelijke halsslagader (carotis communis).

Weefselkarakterizering m.b.v. ultrageluid is al bekend voor het opsporen van afwijkingen in grote organen zoals de lever en de milt (tumoren, hepatitis, levercirrose). Ook in het oog kan men verschillende typen weefsel op die manier onderscheiden. De verzwakking van ultrageluidspulsen door weefsels is afhankelijk van de frequentie van het geluid. Deze frequentie afhankelijkheid is op haar beurt weer typerend voor verschillende weefsels of afwijking van het normale weefsel (tumoren, vet-en collageenophoping etc.). Bij de analyse van de signalen van een bloedvatwand, heeft men echter niet te maken met signalen die over een grote diepte hetzelfde karakter hebben, maar men moet het stellen met een beperkt aantal reflecties van de verschillende overgangen tussen de lagen van een bloedvatwand (zie figuur 1.2). De manier waarop in dit proefschrift ultrageluidssignalen wiskundig worden beschreven kan men vinden in hoofdstuk 2. Het probleem van gereflecteerde ultrageluidssignalen, is dat de sterkte van de reflectie afhankelijk is van de positie en de rorm van het reflecterende vat in de ultrageluidsbundel. Deze verschijnselen zijn onderzocht in de hoofdstukken 3 en 4 . In hoofdstuk 3 werd daartoe een methode beschreven waarmee het mogelijk is om de parameters, die het geluidsveld van een transducent bepalen, te schatten uit metingen van de sterkte van het ultrageluidsveld in een proefopstelling in een waterbak. De parameters die op deze manier werden bepaald werden in hoofstuk 4 gebruikt in het model waarmee de vervorming van een ultrageluidspuls na reflectie op een oppervlak kan worden 
afgeschat. Unt de resultaten van hoofdstuk 4 bljkt dat de vervorming van ultrageluidspulsen het minst erg is als de reflector (bloedvatwand) zich in het verre veld van de transducent bevindt. Ook is de vormverandering van zgm. smalbandige pulsen (landurige pulsen met 1 centrale frequentie) veel minder dan die van breedbandige (korte) pulsen.

Mede daarom, en omdat de verhouding tussen de sterkte van het zuivere signaal en de verstoringen (ruis) veel beter is voor smalbandige pulsen is in dit proefschrift (hoofdstuk 5) voorgesteld $\mathrm{om}_{i,}$ in plats van de gebruikelijke breedbandige korte puls, een aantal pulsen uit te zenden, die ieder een verschillende frequentie hebben. Deze pulsen hebben een lange duur, wat echter tot gevolg heeft dat het niet meer mogelijk is om structuren die dicht bij elkaar liggen te onderscheiden. Dit probleem kan worden opgelost door middel van deconvolutie van de ontvangen signalen. Deconvolutie betekent in feite dat men probeert uit te vinden waar de verschillende reflectoren (weefsellagen) zich bevinden die het waargenomen signaal hebben veroorzaakt, en ook hoe sterk de reflectie van de verschillende weefsellagen is. Hiervoor bestat een zeer eenvoudige methode, genaamd Wiener-inverse filtering, die echter bij gebruik van smalbandige (langdurige) signalen leidt tot artefacten. Er verschijnen "reflectoren" in het gedeconvolueerde signaal die er in werkelijkheid niet zijn. Door ook de resultaten te gebruiken van een uitgezonden puls die iets langer of korter van duur is, kan men de artefacten van het echte signaal onderscheiden en ook onderdrukken.

Bij de resultaten van hoofdstuk 5, waarin gebruik was gemaakt van op de computer nagebootste signalen, bleek al dat het negeren van de karakteristiek van het ultrageluidssysteem leidt tot fouten. Deze blijken te kunnen worden verminderd indien mett op de hoogte is van de zogenaamde overdrachtsfunctie van het ultrageluidssysteem (hoofstuk 6).

In hoofdstuk 7 tenslotte werd de methode getest op 4 jongere mensen en 4 oudere mensen. Deze laatsten ware patienter van de afdeling Neurologie van het AZM in Maastricht. Van deze mensen kon worden verwacht dat er veranderingen in de samenstelling van de bloedvatwand aanwezig zijn. Uit de resultaten bleek inderdaad, dat de frequentie afhankelijke verzwakking gemiddeld genomen flink verschilde tussen de jonge en de oudere personen. Het bleek echter ook, dat de waarden voor een en dezelfde persoon flinke variaties vertoonden. Dit ligt waarschijnlijk aan de effecten zoals die berekend werden in hoofdstuk 4: de waargenomen sterkte van een reflectie is sterk afhankelijk van de vorm en plaats van de weefsellagen van de bloedvatwand. Verder werd in hoofstuk 7 aangetoond dat de beelden die men na bewerking van smalbandige pulsen kan maken niet in kwaliteit onderdoen voor de gebruikelijke beelden die worden verkregen na uitzending wan korte pulsen. Omdat de beelden echter frequentie-specifiek zijn, kan deze techniek mogelijk leiden tot verbetering van de weefselkarakterizering in grote organen.

Verschillende voor- en nadelen van de in dit proefschrift beschreven methoden en technieken werden besproken in hoofdstuk 8 . Daar worden ook aanbeve- 
lingen gedaan om de variatie in de gemeten verzwakkingswaarden te reduceren. Indien de beeldkwaliteit van de conventionele scanner wordt verbeterd, kleine transducenten worden gebruikt, en als de metingen vele malen worden herhaald op hetzelfde moment van de hartcyclus, kan de in dit proefschrift beschreven methode bijdragen tot het onderzoek van de vroege stadia van atherosclerotische afwijkingen en de mogelijke invloed van diverse risicofactoren of het ontstaan van afwijkingen in de boedvatwand. Samen met de resultaten van metingen van de mechanische eigenschappen en de dikte van de vaatwand, kan de beschreven methode mogelijk ook leiden tot vroegtijdiger diagnose.

\section{PUBLICATIES}

F.M.J. Linssen and A.P.G. Hoeks

Transducer characterization from pressure amplitude distribution measurements using a Kalman filter as parameter estimation algorithm.

Ultrasonic Imaging 12 (1990) 309-323

Frans Linssen and Arnold Hoeks

Computation of the signal distortion of reflections from specular reflectors by impullse ray modeling.

Journal of the Acoustical Sociely of America (accepted for publication)

F.M.J. Linssen, A.P.G. Hoeks and P.J. Brands

The use of narrowband ultrasound in the characterization of tissue in a thin layer.

Phys. Med. Biol. 36 (1991) 1319-1330

F.M.J. Linssen and A.P.G. Hoeks

Multi-frequency narrowband tissue characterization

Proceedings of the IEEE 1990 Ultrasonics Symposium 


\section{NAWOORD}

De totstandkoming van dit proefschrift is uiteraard aan een groter aantal mensen toe te schrijven dan alleen de auteur. Natuurlijk in de eerste plaats de bedenker van het project en tevens mijn begeleider Arnold Hoeks. Hij heeft een uitgebreide kennis op het gebied van ultrageluid en signaalverwerking, waarop ik altijd kan terugvallen. Dan natuurlijk Peter Brands die steeds weer klaar stond om mijn bedenksels in electronische hardware om te zetten (ik geef toe, ik ben lastig). Mijn promotor, Arie Hasman, heeft tenslotte de nodige onduidelijkheden en het vakjargon uit het proefschrift weten weg te werken, waarmee het ook voor niet-ultrageluid mensen leesbaar(der) is geworden. Ook de samenhang en de consistentie van het proefschrift als geheel heeft hij in goede banen geleid. Verder is er natuurlijk Rob Reneman, die in de eerste jaren vooral de grote man op de achtergrond was, uit wiens zucht naar kennis het geheel voortgesproten is. In de laatste fase heeft hij de nodige punten op de i's gezet wat betreft de fysiologische achtergrond en ook de historie van het ultraguidsonderzoek van bloedvaten. De metingen aan de proefpersonen zijn uitgevoerd door Tiny van Merode, en mijn dank gaat ook uit naar de proefpersonen (stagiaires en dienstweigeraars die niet al te hard tegenstribbelden als ze bij hun nekvel gevat werden). Rosy Hansen heeft de artikelen die ten grondslag liggen aan de hoofdstukken 3 en 4 uitgewerkt. In de allerlaatste fase hebben ook de leden van de leescommissie hun steentje bijgedragen.

De mensen die niet hebben bijgedragen aan het werk, maar wel aan een positieve werk-en leefsfeer, zijn wellicht net zo belangrijk (zo niet belangrijker). Hierbij denk ik in de eerste plaats natuurlijk aan Monique, zonder wie dit proefschrift waarschijnlijk niet eens was geschreven en verder aan de mensen in de Waberstraat, waar ik tijdens de eerste jaren in Maastricht met veel plezier gewoond heb. Natuurlijk denk ik nu niet aan de mensen die ik vergeet, maar ze worden toch bedankt. 


\section{CURRICULUM VITAE}

- Geboren 31 December 1962

- Atheneum-B aan het Bisschoppelijk College te Echt 1975-1981

- Werktuigbouwkunde aan de Technische Universiteit Eindhoven 1981 1987

- Assistent In Opleiding aan de Rijksuniversiteit Limburg, vakgroep Biofysica $1988-1992$ 